\title{
Recent progress in the description of positron scattering from atoms using the Convergent Close-Coupling Theory
}

\author{
A S Kadyrov and I Bray \\ Curtin Institute for Computation and Department of Physics, Astronomy and Medical Radiation Science, \\ Kent Street, Bentley, Perth, Western Australia 6102, Australia
}

(Dated: September 15, 2016)

\begin{abstract}
Much progress in the theory of positron scattering on atoms has been made in the ten years since the review of Surko et al. [1]. We review this progress for few-electron targets with a particular emphasis on the two-centre convergent close-coupling and other theories which explicitly treat positronium (Ps) formation. While substantial progress has been made for Ps formation in positron scattering on few-electron targets, considerable theoretical development is still required for multielectron atomic and molecular targets.
\end{abstract}

PACS numbers: $34.80 .-\mathrm{i}$ 34.80.Lx 34.80.Uv 34.10.+x

\section{CONTENTS}

I. Introduction
A. Positron scattering
1. atomic hydrogen
2. helium
3. alkali metals
4. magnesium
5. inert gases
6. molecular hydrogen

B. Antihydrogen formation

II. Theory
A. Atomic hydrogen
B. Helium
C. Alkali metals
D. Magnesium and inert gases
E. Molecular hydrogen

III. Recent applications of the CCC theory to positron scattering

A. Internal consistency

14

B. Atomic hydrogen

C. Helium

17

D. Alkali metals

18

E. Magnesium and inert gases

F. Molecular hydrogen

IV. Antihydrogen formation

A. Numerical treatment of the Green's function 26

B. Analytic treatment of the Green's function 26

V. Concluding remarks

Acknowledgments

References

\section{INTRODUCTION}

There have been many reviews of positron physics over the years [1 9]. More recently Laricchia et al. [10] and Chiari and Zecca [1] considered the subject with an emphasis on experimental measurements involving noble gas targets. The related topic of antihydrogen formation has also been thoroughly reviewed 12 15]. Resonances and the closely related bound states of positrons with atoms and molecules has also been extensively discussed [1[3, 16 [18].

This work concentrates on the progress in application of theoretical methods to scattering processes in a quantum few-body system involving positrons as projectiles and multi-electron atomic targets with explicit treatment of positronium formation. Particular emphasis is on the developments taken place since the comprehensive review of positron physics by Surko et al. [1]. It begins by describing the currently available theories of low-energy positron collisions with atoms and simple molecules. Then it describes the development and application of the two-centre convergent close-coupling method, which explicitly treats the Ps-formation processes.

\section{A. Positron scattering}

Developments in positron physics have resulted in several technologies in medicine and material science. In medicine, the use of the positron emission tomography (PET) scanners help to make diagnoses of cancer detection and of certain brain function disorders. Material science uses positron annihilation lifetime spectroscopy (PALS), to analyze and design specific materials. The critical component is positronium (Ps) formation with its annihilation providing the key signature of its origin. Ps is a short-lived exotic atom of a bound positron-electron pair that has similar structure to atomic hydrogen.

Scattering experiments are the main tool of modern physics to learn about the structure of matter. By ana- 
lyzing collision products we can extract useful information about the objects being studied. Historically, ordinary matter particles like electrons and protons were predominantly used as scattering particles in experimental atomic and molecular physics. With the development of positron and antiproton beams, studies of interactions of these particles with matter became possible.

The last decade has seen significant progress in lowenergy trap-based positron beams [1, 19]. New highresolution experimental measurements have been performed for a range of atomic and molecular targets including $\mathrm{He}$ [20], Ne and $\operatorname{Ar}$ 21, 22], Xe [23], Kr 24, 25] $\mathrm{H}_{2}$ [26] and $\mathrm{H}_{2} \mathrm{O}$ [27]. The development of positron beams motivated novel experimental and theoretical studies. Particularly important are the positroniumformation cross sections, see the recent recommendations of Machacek et al. 28]. In addition, interest has been motivated by possible binding of positrons to atoms [3].

Theoretical description of electron-impact ionisation (and excitation) processes has seen significant progress in recent years due to the development of various highly sophisticated methods including the exterior complex scaling (ECS) 29 31], R-matrix with pseudo-states (RMPS) 32 35], time-dependent close-coupling (TDCC) 36] and convergent close coupling (CCC) 37 39]. A review of electron-induced ionisation theory has been given by Bray et al. [40]. Such problems are examples of a class where there is only one "natural" centre, namely the atomic centre. All coordinates are readily written with the origin set at the atomic centre. Yet there are many atomic collision systems of practical and scientific interest that involve at least two centres, such as the positronhydrogen scattering system. This is a three-body system where all the particles are distinguishable, and which allows for their rearrangement. Here we have two "natural" centres, the atomic centre and the positronium $(\mathrm{Ps})$ centre. For positron-hydrogen scattering ionisation now splits into two separate components: the rearrangement process of Ps-formation and the three-body breakup process. A proper formulation of Ps-formation processes requires a combined basis consisting of two independent basis sets for each of the centres which makes theoretical studies considerably more challenging than for electronscattering. Furthermore, the positron-atom system is an ideal prototype of the ubiquitous collision systems such as proton-atom scattering, where charge-exchange processes also require a two-centre treatment, see Abdurakhmanov et al. 41] for example.

Every positron-atom scattering system has an ionisation (breakup) threshold above which an electron may be freely ejected. At $6.8 \mathrm{eV}$ below this threshold is the Ps formation threshold. At higher energies excitation or ionization of the target can take place. In addition, for multi-electron targets there could be many more reaction channels such as multiple ionisation and ionisation-withexcitation. For molecular targets there could be rovibrational excitation and dissociation. Another reaction channel is the positron-electron pair annihilation, which can occur at any scattering energy. In this process the positron collides with one of the target's electrons and annihilates into 2 or 3 gamma rays. Theoretical [42, 43] and experimental studies [4] of the annihilation process have shown that its cross section is up to $10^{5}$ times smaller than the elastic-scattering cross section. Therefore the annihilation channel is often omitted from scattering calculations. The elastic scattering, excitation, Ps formation and ionisation are the dominant channels of primary interest.

\section{1. atomic hydrogen}

The first theoretical studies of positron scattering from atoms date back to the 1950s, when Massey and Mohr [45] used the first Born approximation (FBA) to describe Ps-formation in $\mathrm{e}^{+}-\mathrm{H}$ collisions. The Born method is based on the assumption that the wavefunction for the scattering system can be expanded in a rapidly convergent series. This approximation consists in using plane waves to describe the projectile and scattered particles. The Born approximation is reliable when the scattering potential is relatively small compared to the incident energy, and thus is applicable only at high energies. Therefore this method is mainly focused on high-energy excitation and ionization processes.

One of the most successful methods applied to the lowenergy $\mathrm{e}^{+}-\mathrm{H}$ elastic-scattering problem is the Kohn variational method. The method was initially developed for scattering phase shifts in nuclear reactions by Kohn [46]. Later this method was extended to positron-hydrogen scattering by Schwartz [47]. A detailed description of the method was given by Armour and Humberston [48]. The method is based on finding the form of the functional (called Kohn functional) involving the phaseshift as a parametric function of the total wavefunction. Requiring the functional to be stationary with respect to the variations of the parameters, generates equations for the linear parameters. The phaseshifts can be accurately obtained by performing iterative calculations and finding the values of the nonlinear parameters that make the functional stationary.

The many-body theory of Fetter and Walecka [49] utilizes techniques that originated from quantum field theory. Using the Feynman-diagram technique the perturbation series in the interaction between particles can be written in an intuitive way. When it is applied to positron-atom scattering, however, difficulties arise due to necessity to take into account (virtual) Ps formation. However, a finite number of perturbation-theory terms cannot describe a bound Ps state. Gribakin and King [50] developed a sophisticated method based on the manybody perturbation theory. They used an approximation by considering virtual Ps formation only in the ground state. The calculations with this method showed that for elastic scattering of positrons on hydrogen (and helium atoms), the virtual Ps-formation contribution was almost 
$30 \%$ and $20 \%$ of the total correlation potential, respectively. Gribakin and Ludlow [51] have further improved the method by introducing the techniques for the exact summation of the electron-positron ladder diagram series. The method was applied to $\mathrm{e}^{+}-\mathrm{H}$ scattering below the Ps-formation threshold, and resulted in good agreement with accurate variational calculations.

The momentum-space coupled-channel optical (CCO) potential method was first developed for electron-atom scattering in the 1980s by McCarthy and Stelbovics [52, 53]. The method relies on constructing a complex equivalent local potential to account for the ionization and the Ps-formation channels. The CCO method gave excellent ionization [54], total and Ps formation [55] cross sections for positron scattering on hydrogen. Ma et al. [56] used the CCO method to study excitation of atomic hydrogen from the metastable $2 \mathrm{~s}$ state.

Following Macri et al. [57] Jiao et al. [58] developed the so-called two-center two-channel eikonal final statecontinuum initial distorted-wave model to calculate Ps formation in the ground and the lowest excited states. They also presented a $n^{-s}$ scaling law for formation of $\operatorname{Ps}(n)$ cross sections on the entire energy range with $s$ varying as a function of the positron incident energy.

A hyperspherical hidden crossing (HHC) method has been applied to positron-impact ionization of hydrogen near the threshold by Jansen et al. [59]. They have calculated the ionization cross-section for $\mathrm{S}, \mathrm{P}$ and $\mathrm{D}$-waves. The HHC has also been used to calculate partial-wave Ps $(1 s)$-formation cross sections for low-energy positron collisions with $\mathrm{H}, \mathrm{Li}$ and $\mathrm{Na}$ atoms [60].

One of the most sophisticated and commonly used methods is the close-coupling (CC) formalism, which is based on the expansion of the total wavefunction using the target-state wavefunctions. Substitution of this expansion into the Schrödinger equation yields coupled differential equations in coordinate space, or LippmannSchwinger integral equations for the T-matrix in momentum space. By solving these equations the transition amplitudes are obtained for all open channels. Considerable pioneering work in this field has been done by Hewitt et al. [61], Higgins and Burke 62], Mitroy [63] and Walters et al. [64], who demonstrated the success of using two-centre expansions consisting of Ps and atomic states.

Here and below when we discuss close-coupling calculations we denote the combined two-centre basis, used to expand the total scattering wavefunction, as $(N, M)$, where $N$ is the number of atomic (negative-energy) eigenstates and $M$ is the number of positronium eigenstates. We also use a bar to indicate (negative- and positiveenergy) pseudostates. For instance, $\operatorname{CC}(\bar{N}, M)$ refers to close-coupling calculations with a combined basis made of $N$ pseudostates for the atomic centre supplemented by $M$ Ps eigenstates.

Higgins and Burke [62] performed the first accurate two-state $\mathrm{CC}(1,1)$ calculation. This work known as the static-exchange approximation showed a giant spurious resonance near $40 \mathrm{eV}$ incident positron energy. Absence of such a resonance was demonstrated by Kernoghan et al. [65] using a larger $\mathrm{CC}(\overline{9}, \overline{9})$ calculation that included $s, p$, and $d$-type pseudostates for both centres. However, the $\operatorname{CC}(\overline{9}, \overline{9})$ gave new spurious resonances above the ionization threshold. An energy-averaging procedure was used to get smooth results for the cross sections to get rid of the pseudoresonances.

Considerable progress in the description of $\mathrm{e}^{+}-\mathrm{H}$ has been made by Mitroy [63] by using the close-coupling approach. Mitroy and Ratnavelu [66] have performed convergence studies for the full positron-hydrogen problem at low energies. Below the ionization threshold they showed good agreement of sufficiently large pseudostate close-coupling calculations and the benchmark variational calculations of Ps formation by Humberston 67].

The convergent close-coupling (CCC) method was first developed for $\mathrm{e}^{-}-\mathrm{H}$ scattering by Bray and Stelbovics 68]. Its modification to positron scattering in a onecentre approach was trivial in that electron exchange was dropped and the interaction potentials changed sign [69, 70$]$. The CCC method with a $(\bar{N}, 0)$ basis, i.e. a single atomic-centre expansion without any Ps states, gave very good results for the total, elastic, excitation and ionization cross sections at higher incident energies where the Ps-formation cross section is small allowing for distinction between two experimental data sets [70]. The $\mathrm{CCC}$ calculations showed no pseudoresonances so long as a sufficiently large basis was taken.

Following the success of the large single-centre CCC calculations, Kernoghan et al. [71] and Mitroy [72] used a large basis for the atomic centre supplemented by a few eigenstates of Ps. Calculations with the $(\overline{28}, 3)$ and $(\overline{30}, 3)$ bases, made of a large atomic basis similar to that of Bray and Stelbovics [70] and the three lowest-lying eigenstates of Ps, gave results significantly better than those from the $(\overline{9}, \overline{9})$ basis 65$]$.

The CCC method with a $(\bar{N}, \bar{M})$ basis was developed by Kadyrov and Bray [73] to study convergence in two-centre expansions. This was applied to positron scattering on hydrogen within the S-wave model retaining only $s$-states in the combined basis. This work for the first time demonstrated the convergence of the (nonorthogonal) two-centre expansions. The convergence in all channels was only possible when two independent near-complete Laguerre bases are employed on both of the centres. Interestingly, the total ionisation cross section had two independently converged components. One component was coming from the atomic centre and represented direct ionisation of the hydrogen atom, the other came from the Ps centre and represented Ps formation in the continuum. The convergence in the case of the full positron-hydrogen scattering problem was demonstrated by Kadyrov and Bray 74]. The CCC calculations with such a combined two-centre basis have shown very good agreement with the experimental measurements of Zhou et al. [75] and Jones et al. 76]. 


\section{2. helium}

Theoretical investigations of positron scattering from helium has an additional challenge due to the complexity of the target structure. In multi-electron targets twoelectron excitation (or ionization with excitation) channels are usually excluded. This is a good approximation as the contribution of these channels is typically two orders of magnitude smaller than the corresponding oneelectron excitation processes [77].

First calculations of $\mathrm{e}^{+}-\mathrm{He}$ scattering have been performed by Massey and Moussa [78] in the FBA. They used only the ground states for He and Ps and obtained cross sections for elastic scattering and Ps formation in its ground state. Their study highlighted the importance of the Ps-channel coupling with the elastic channel and thereby motivated further studies. Another extensive study based on the Born approximation was presented by Mandal et al. 79], to estimate Ps-formation cross section in arbitrary S-states. From the FBA studies it became clear that more sophisticated approaches to the problem were required.

The distorted-wave Born approximation (DWBA) results are obtained by using distorted wavefunctions in first-order calculations. This method can give more accurate results than the FBA down to lower energies. Studies utilizing the DWBA by Parcell et al. [80, 81] were applied to the helium $2^{1} S$ and $2^{1} P$ excitations by positrons in the energy range from near the threshold up to $150 \mathrm{eV}$. Although the agreement with the experimental data was not very satisfactory, the method indicated the importance of the inclusion of the polarization potential in the excitation channels at low energies. The most systematic study of the ionisation process within the framework of DWBA was carried out by Campeanu et al. [82, 83. They used Coulomb and plane waves and also included exchange effects. They obtained good agreement with the experimental results of Fromme et al. 84, Knudsen et al. 85] and Moxom et al. 86. over the energy range from near-threshold to $500 \mathrm{eV}$. However, the most important and difficult channel, Ps formation, was not included in the early DWBA studies.

Srivastava et al. [87] calculated the differential and total cross sections for the excitation of the helium $2^{1} S$ state using the second-order DWBA method. Another DWBA method including Ps channels has been reported by Sen and Mandal [88] for intermediate to high scattering energies. They have calculated Ps-formation cross section and achieved good agreement with available experimental data above $60 \mathrm{eV}$. However, their results were not accurate for Ps formation below $60 \mathrm{eV}$. Considering the fact that Ps formation starts at $17.8 \mathrm{eV}$ and reaches its maximum around $40 \mathrm{eV}$, the applicability of this method is quite limited.

Amusia et al. [89] applied the random-phase approximation (RPA), based on many-body theory [49], to positron-helium scattering at low energies. By using an approximate account of virtual Ps formation they ob- tained good agreement with the elastic-scattering experimental data of Jaduszliwer and Paul [90] and Canter et al. 91] at the lowest energies. A similar RPA method was used by Varracchio 92 to calculate positron impact excitation of $\mathrm{He}$ into $2^{1} \mathrm{~S}$ and $2^{1} \mathrm{P}$ states. As mentioned above, Gribakin and King [50] developed a more sophisticated method based on the many-body perturbation theory. The calculations with this method showed that the contribution from virtual Ps formation was significant. Applications of the method to various atomic targets were reported by Dzuba et al. 93. and Gribakin and King [94].

The Kohn variational method was first applied to positron-helium collisions by Humberston et al. 95 98]. A comprehensive study of positron-helium scattering with the Kohn variational method was given by Reeth and Humberston 99]. They obtained very accurate cross sections at low energies. However, agreement with the experimental results of Mizogawa et al. [100] and Stein et al. 101] for Ps-formation cross section was qualitative, with a similar energy dependence but with almost $25 \%$ difference in magnitude. Nevertheless, very good agreement was obtained for the total cross section below the Ps-formation threshold.

The CCO method, mentioned earlier, was applied to positron scattering on helium by Cheng and Zhou [102]. They calculated the total and Ps-formation cross sections from the Ps-formation threshold to $500 \mathrm{eV}$. The calculated results agreed well with the corresponding experimental data except for the data of Griffith and Heyland [9] for the total cross section in the energy range from 50 to $100 \mathrm{eV}$. McEachran et al. 103 applied a polarized-orbital approximation method to low-energy elastic positron-helium scattering and obtained good agreement with the experimental results. Other calculations using optical potentials were presented by Tancić and Nikolić 104] and by Gianturco and Melissa [105] for slow-positron scattering from helium. Elastic-scattering cross sections of both reports were in good agreement with experimental data. In general, the optical-potential methods proved to be useful for calculations of total cross sections. They are problematic, however, when applied to more detailed cross sections like target excitation and Ps formation in excited states.

Schultz and Olson [106] utilized the classical-trajectory Monte-Carlo (CTMC) technique to model positron scattering. The method is described fully for ion-atom collisions by Abrines and Percival [107] and by Olson and Salop [108]. Using this technique, Schultz and Olson [106] calculated differential ionization cross section for positron-helium and also positron-krypton collisions. The main advantage of this method is that it can describe dynamic effects occurring in collisions. For instance, the CTMC calculations showed that the probability of positron scattering to large angles after ionising the target, may be comparable, or even much greater than, the probability of positronium formation. They suggested that the disagreement between theory and ex- 
periment above $60 \mathrm{eV}$ might be resolved by accounting for the flux in the experiments measuring positronium formation due to positrons scattered to angles that allow them to escape confinement.

Tökési et al. [109] also applied the CTMC method to helium ionization by positron impact. They obtained good agreement with experimental data of Fromme et al. [84]. Results of CTMC reported by Schultz and Olson [106] overestimate the recent experimental data by Caradonna et al. 110] for the Ps-formation cross section below $60 \mathrm{eV}$. This questions the applicability of the classical trajectory approach to positron scattering at low and intermediate energies.

A very comprehensive study of positron-helium scattering using the close-coupling method was carried out by Campbell et al. [111]. They used two kinds of expansions, the first one consisting of 24 helium eigen- and pseudostates and the lowest three Ps eigenstates, and the second one with only 30 helium eigen- and pseudostates. The helium-target structure was modeled using a frozen-core approximation, which can produce good excited states, but a less accurate ground state. The atomic pseudostates were constructed using a Slater basis. For the 27-state approximation, only results in the energy range above the positronium-formation threshold were given. Results for lower energies were unsatisfactory, and it was suggested that this might be due to the lack of convergence from the use of an inaccurate helium ground-state wavefunction. The total cross sections from both the 27- and 30-state approaches agreed well with the experimental results of Stein et al. [101], Kauppila et al. [112] and Mizogawa et al. [100] for the energy range above the threshold of positronium formation. For lower energies, qualitative agreement was obtained in terms of the shape and the reproduction of the Ramsauer-Townsend minimum near $2 \mathrm{eV}$, while the theoretical results were a factor of 2 larger than the experimental data. The Psformation cross section from the 27-state approximation was in good agreement with the experimental data of Moxom et al. [86] up to about $60 \mathrm{eV}$ and with the data of Fornari et al. [113] and Diana et al. [114] up to $90 \mathrm{eV}$. Above $100 \mathrm{eV}$ the calculations were much lower than the experimental data of Diana et al. [114] and Fromme et al. [84] while being closer to the data of Overton et al. [115].

Another close-coupling calculation by Hewitt et al. [61, 116], using a few helium and positronium states with a one-electron description of the helium atom, showed less satisfactory agreement with the experimental data. Chaudhuri and Adhikari [117 119] performed calculations using only 5 helium and 3 positronium states in the expansion. Their results for Ps formation agreed well with the experimental results by Moxom et al. [86] at energies near the Ps threshold and displayed a better agreement with the data of Overton et al. [115] at the higher energies. However, the theoretical results were much lower than the experimental data at energies near the maximum of the cross section.

Igarashi et al. [120] applied the hyper-spherical CC ap- proach to the problem. However, they also considered helium as a one-electron target, and thus the excitation and Ps-formation cross sections were multiplied by factor of 2. Satisfactory results were obtained for the total Ps-formation and $\mathrm{He}\left(2^{1} \mathrm{~S}\right)$ and $\mathrm{He}\left(2^{1} \mathrm{P}\right)$ excitation cross sections. The method was not able to describe low-energy scattering mainly because a one-electron approach to helium is not realistic at low energies.

Despite the obvious advantages of the abovementioned close-coupling calculations in handling many scattering channels simultaneously, none was able to describe low-energy elastic scattering. In addition, the presence of pseudo-resonances in cross sections below the ionization threshold [111, 116] indicated that there was room for improvement. The use of the frozen-core He states also needed some attention as this yielded an inaccurate result for the ground state of helium.

The first application of the single-centre CCC method to positron scattering on helium was made by $\mathrm{Wu}$ et al. [121, 122], using very accurate helium wavefunctions obtained within the multi-core approximation. Very accurate elastic cross section was obtained below the Psformation threshold by using orbitals with very high angular momenta. It was suggested that the necessity for inclusion of very high angular-momentum orbitals was to mimic the virtual Ps-formation processes. The method also gave accurate results for medium to high-energy scattering processes except that it was unable to explicitly yield the Ps-formation cross section. Interestingly, the method was not able to produce a converged result at the Ore-gap region (where only the elastic and Ps-formation channels are open), which we shall discuss later in sec. IIIA. Comparison of the frozen-core and the multi-core results showed that the frozen-core wavefunctions lead to around $10 \%$ higher cross sections. Wu et al. 121, 122] demonstrated that single-centre expansions can give correct results below the Ps-formation threshold and at high energies where the probability of Ps formation is small, but for the full solution of the problem inclusion of the Ps centre into expansions was required.

The initial application of the two-centre CCC approach to the problem was within the frozen-core approximation 123, 124. It was then extended to a multi-core treatment [125]. While generally good agreement was found with experiment from low to high energies certain approximations made need to be highlighted. The key problematic channels are those of the type Ps- $\mathrm{He}^{+}$. Electron exchange between $\mathrm{Ps}$ and $\mathrm{He}^{+}$is neglected. Excitation of $\mathrm{He}^{+}$is also neglected. While these may seem reasonable approximations for the helium target due to its very high ionisation threshold $(24.6 \mathrm{eV}$ for $\mathrm{He}$ and $54.4 \mathrm{eV}$ for $\mathrm{He}^{+}$), they become more problematic for quasi two-electron targets such as magnesium, discussed below.

Positron scattering from the helium $2^{3} \mathrm{~S}$ metastable state has been theoretically studied for the first time by Utamuratov et al. 126] at low and intermediate energies. Converged results for the total, Ps-formation and 
breakup cross sections have been obtained with a high degree of convergence. The obtained cross sections turned out to be significantly larger than those for scattering from the helium ground state.

\section{3. alkali metals}

Alkali atoms have an ionisation threshold that is lower than $6.8 \mathrm{eV}$. Consequently, for positron scattering on alkalis the elastic and Ps-formation channels are open at all incident-positron energies. For this reason, theory has to treat appropriately the "competition" for the valence electron between the two positively charged centers, the singly-charged ionic core and the positron.

Positron scattering on the lithium target was investigated by Ward et al. [127], Sarkar et al. [128], Khan et al. [129] using a one-center expansion. However, convergence was poor due to the absence of Ps-formation channels [130]. Two-center expansion was employed by Guha and Ghosh 131, Basu and Ghosh 132, Abdel-Raouf [133], Hewitt et al. 134], McAlinden et al. [130] and Le et al. [135]. As expected, these approaches gave better agreement with the experiment. For positron-lithium case the two-center CCC approach to positron collisions with lithium was reported by Lugovskoy et al. [136]. This is the most comprehensive study of the problem on an energy range spanning six orders of magnitude. While convergence was clearly established, and agreement with experiment for the total Ps-formation cross section [137] is satisfactory, smaller experimental uncertainties would be helpful to provide a more stringent test of the theory.

Positron scattering from atomic sodium has been intensively studied for more than two decades. The first theoretical calculations relied on simple two-center decomposition of the system wavefunction with only the ground states of sodium and Ps atoms taken into account 133, 138, 139]. Then, Hewitt et al. [140] conducted more complex close-coupling calculations, adding several low-lying excited states for each positively-charged center. The obtained results turned out to be in reasonably good agreement with experimental data for both total [141, 142 and Ps-formation 143] cross sections.

Further enlargement of the number of channels in the close-coupling calculations revealed that the theoretical Ps-formation cross sections [111, 144, 145] deviated systematically from the experimental results for low impact energies. The experiment showed that the Ps-formation cross section became larger with decreasing energy while the most refined theoretical calculations utilizing different methods of solution predicted consistently the opposite.

To resolve the discrepancy Surdutovich et al. 137 conducted the experimental study on Ps formation in positron collisions with $\mathrm{Li}$ and $\mathrm{Na}$ atoms. This experiment confirmed the earlier results of Zhou et al. [143]. The authors managed to extend the impact-energy range down to $0.1 \mathrm{eV}$ where the discrepancy between the the- ory and experiment was even larger for sodium. In striking contrast, for lithium the reasonable agreement of the measured cross section with the theoretical predictions was obtained with the use of the same methodology. Ke et al. [146] applied the optical-potential approach. They found that their theoretical cross section increases with the decrease in the impact energies below $1 \mathrm{eV}$, but faster than the experimental results. Unfortunately, this result was obtained with the use of some approximations, whose validity were not analyzed. It would be instructive if the same optical-potential approach was applied to the case of positron scattering on lithium.

Le et al. [135] calculated Ps-formation in positronalkali collisions with the use of the hyper-spherical closecoupling method. Their results support the previous theoretical data 111, 144, 145]. Large two-center CCC calculations of positron scattering by atomic sodium were reported by Lugovskoy et al. [147]. Despite being the most comprehensive to date there was no resolution of the discrepancy with experiment for Ps formation at low energies, which we will highlight in Sec IIID.

While the lighter alkali atoms are well-modeled by a frozen-core Hartree-Fock approximation, or even an equivalent local core potential, the heavier ones become more problematic. With a reduced ionisation threshold positron interaction with the core electrons, either directly or via exchange of the valence and the core electrons, becomes a more important component of the interaction. To the best of our knowledge this has not been addressed to a demonstrable level of convergence by any theory. Nevertheless, assuming that such problems are more likely to be a problem at the higher energies, Lugovskoy et al. [148] considered threshold behaviour of the elastic and Ps-cross sections, and their convergence properties at near-zero energies for $\mathrm{Li}, \mathrm{Na}$ and $\mathrm{K}$. This work confirmed the expected threshold law proposed by Wigner [149], but was unable to resolve the discrepancy with the positron-sodium experiment at low energies. Some earlier studies by Hewitt et al. [140] and McAlinden et al. 150] at low to intermediate energies were performed at a time when convergence was computationally impossible to establish.

Chin et al. 151] further developed the CCO method to study positron scattering on rubidium at intermediate and high energies. They calculated the Ps-formation and total cross sections. Their total cross section results appear to overestimate the experiment.

Though outside the scope of this review we note that the complex scaling method was recently used to study resonance phenomena in positron scattering on sodium [152] and potassium [153].

\section{4. magnesium}

Magnesium can be thought of as a quasi two-electron target with the core electrons being treated by the selfconsistent field Hartree-Fock approach. Positron scat- 
tering on magnesium is particularly interesting due to a large resonance in elastic scattering identified at low energies by Mitroy et al. 154]. This was confirmed, though at a slightly different energy, by the one-centre calculations of Savage et al. [155] which were able to be taken to convergence in the energy region where only elastic scattering was possible. Minor structure differences are likely to be responsible for the small variation in the position of the resonance. Two-centre CCC calculations [156] also reproduced the resonance, but had to make substantial approximations when treating the $\mathrm{Ps}_{\mathrm{s}} \mathrm{Mg}^{+}$interaction. This is even more problematic than in the case of helium discussed above since now we have a multi-electron Hartree-Fock core. Agreement with experiment is somewhat variable, but there are substantial experimental uncertainties, particularly in the Ps-formation cross section. Other theoretical studies of positron-magnesium scattering include those by Gribakin and King [94], Campbell et al. [111], Campeanu et al. [157], Bromley et al. [158], Hewitt et al. [159].

\section{5. inert gases}

Inert gases heavier than helium represent a particular challenge for theory, which is unfortunate because they are readily accessible experimentally [11, 160]. Just the target structure is quite complicated, but some good progress has been made in the case of electron scattering by Zatsarinny and Bartschat [161, 162]. For positron scattering once Ps forms, the residual ion is of the openshell type making full electron exchange incorporation particularly problematic. The relatively high ionisation thresholds for such targets mean that there is always a substantial Ore gap where the Ps-formation cross section may be quite large, but unable to be obtained in onecentre calculations which are constrained to have only elastic scattering as the open channel. Nevertheless, outside the extended Ore gap, formed by the Ps-formation and the ionisation thresholds, one-centre CCC calculations can yield convergent results in good agreement with experiment [163]. There are also first-order perturbative calculations by Campeanu et al. 83., Dzuba et al. [93], Chen et al. [164], Parcell et al. [165] and some based on close-coupling with convergence not fully established, see McAlinden and Walters [166], Gilmore et al. [167].

Green et al. [168] studied positron scattering and annihilation on noble-gas atoms using many-body theory at energies below the Ps-formation threshold. They demonstrated that at low energies, the many-body theory is capable of providing accurate results. Fabrikant and Gribakin [169] used an impulse approximation to describe Ps scattering on inert gases and provided quantitative theoretical explanation for the experimentally-observed similarity between the $\mathrm{Ps}_{\mathrm{S}}$ and electron scattering for equal projectile velocities [170]. According to Fabrikant and Gribakin [169] this happens due to the relatively weak binding and diffuse nature of Ps, and the fact that elec- trons scatter more strongly than positrons off atomic targets.

Poveda et al. 171 developed a model-potential approach to positron scattering on noble-gas atoms based on an adiabatic method that treats the positron as a light nucleus. The method was applied to calculate the elastic cross section below the Ps-formation threshold.

\section{6. molecular hydrogen}

Positron collisions with molecular hydrogen have been studied extensively by various experimental groups over the last 30 years [26, 85, 113, 114, 172 181]. Theoretical studies of this scattering system are challenging because of the complexities associated with the molecular structure and its non-spherical nature. Rearrangement processes add another degree of complexity to the problem. Until recently theoretical studies [48, 182 194] have been focused only at certain energy regions. In addition, there are few theoretical studies which include the Psformation channels explicitly. The first calculations of Ps-formation cross section [182 184, 186] were obtained with the use of the first Born approximation. Biswas et al. [187] used a coupled-static model, which only included the ground states of $\mathrm{H}_{2}$ and Ps. This simple model was until recently the only coupled-channel calculation available. Comprehensive review of the positron interactions with atoms and molecules has been given by Surko et al. [1].

Fedus et al. 195] have recently reported the total cross section for positron scattering from the ground state of $\mathrm{H}_{2}$ below the Ps-formation threshold using density functional theory with a single-center expansion. Their results are in good agreement with recent single-centre CCC calculations of Zammit et al. [196] below 1 eV. Fedus et al. 195] have also performed analysis of experimental and theoretical uncertainties using a modified effective range theory (MERT). They concluded that a practically constant value of the total cross section between $3 \mathrm{eV}$ and the Ps-formation threshold is likely to be an effect of virtual Ps formation.

The recent single-centre CCC calculations of positron scattering on molecular hydrogen by Zammit et al. 196] and antiproton collisions with $\mathrm{H}_{2}$ by Abdurakhmanov et al. [197, 198] have shown that the CCC formalism can also be successfully applied to molecular targets. In order to obtain explicit Ps-formation cross section a twocentre approach is required, with the first attempt presented by Utamuratov et al. [199]. They found some major challenges associated with the $\mathrm{Ps}_{-} \mathrm{H}_{2}^{+}$channel. Some severe approximations were required in order to manage the non-spherical $\mathrm{H}_{2}^{+}$ion. Nevertheless, some good agreement with experiment was found, see Sec. IIIF but considerably more work is required. 


\section{B. Antihydrogen formation}

A major application of positron-hydrogen scattering is to provide a mechanism for antihydrogen formation. The idea is fairly simple, with some accurate calculations performed quite some time ago [200]. The basic idea is to time reverse the Ps-formation process to hydrogen formation from Ps scattering on a proton, and then to use the resultant cross sections for the case where the proton is replaced by an antiproton, and hence forming antihydrogen

$$
e^{+}+\mathrm{H} \leftrightarrow \mathrm{Ps}+p^{+} \equiv e^{-}+\overline{\mathrm{H}} \leftrightarrow \mathrm{Ps}+p^{-} .
$$

The advantage of antihydrogen formation via this process is that it is exothermic and so the cross section tends to infinity as the relative energy goes to zero [149]. This behaviour is enhanced in the case of excited states with degenerate energy levels 201]. Antihydrogen formation is presently particularly topical due to several groups (AEgIS [202 204], GBAR 205 207], ATRAP [208], ASACUSA [209] and ALPHA 210]) attempting to make it in sufficient quantity in order to perform spectroscopic and gravitational experiments. Kadyrov et al. [211] provided CCC results for Ps energy starting at $10^{-5} \mathrm{eV}$, which suffices for currently experimentally accessible energies of around $25 \mathrm{meV}$. Recent calculations of the cross sections for these processes will be discussed in Sec. IV.

In this section we gave a general historical overview of various theoretical developments related to positron scattering on atomic targets and the $\mathrm{H}_{2}$ molecule. In the next section we consider in some detail basic features of the coupled-channel formalism mainly in the context of convergent close-coupling method and discuss the latest results.

\section{THEORY}

\section{A. Atomic hydrogen}

Here we describe basics of the close coupling approach based on the momentum-space integral equations. We consider the simplest case of scattering in a system of three particles: positron (to be denoted $\alpha$ ), proton $(\beta)$ and electron $(e)$. Let us also call $\alpha$ the pair of proton with electron, $\beta$ - positron with electron and $e$ - positron with proton. We neglect spin-orbit interactions. In this case spacial and spin parts of the total three-body wavefunction separate. The latter can be ignored as it has no effect on scattering observables. The spacial part of the total three-body scattering wavefunction satisfies

$$
(H-E) \Phi=0,
$$

where

$$
H=H_{0}+V_{\alpha}+V_{\beta}+V_{e} \equiv H_{0}+V
$$

is the full Hamiltonian, $H_{0}$ is the three-free-particle Hamiltonian, $V_{i}$ is the Coulomb interaction between particles of pair $i(i=\alpha, \beta, e)$. The total Hamiltonian can also be expressed in the following way

$$
H=H_{\gamma}+\frac{q_{\gamma}^{2}}{2 M_{\gamma}}+\bar{V}_{\gamma},
$$

where $H_{\gamma}$ is the Hamiltonian of the bound pair $\gamma, \boldsymbol{q}_{\gamma}$ is the momentum of free particle $\gamma$ relative to c.m. of the bound pair, $M_{\gamma}$ is the reduced mass of the two fragments and $\bar{V}_{\gamma}=V-V_{\gamma}$ is the interaction potential of the free particle with the bound system in channel $\gamma(\gamma=\alpha, \beta)$.

Coupled-channel methods are based on expansion of the total wavefunction $\Phi$ in terms of functions of all asymptotic channels. However, since the asymptotic wavefunction corresponding to 3 free particles has a complicated form [212, 213], this is not practical. Therefore, we approximate $\Phi$ by expansion over some negative and discrete positive energy pseudostates of pairs $\alpha$ and $\beta$ especially chosen to best reproduce the corresponding physical states. Suppose we have some $N_{\mathrm{H}}$ pseudostates in pair $\alpha$ and $N_{\mathrm{Ps}}$ in pair $\beta$ satisfying the following conditions

$$
\left\langle\psi_{\alpha^{\prime}} \mid \psi_{\alpha}\right\rangle=\delta_{\alpha^{\prime} \alpha}, \quad\left\langle\psi_{\alpha^{\prime}}\left|H_{\alpha}\right| \psi_{\alpha}\right\rangle=\delta_{\alpha^{\prime} \alpha} \varepsilon_{\alpha}
$$

and

$$
\left\langle\psi_{\beta^{\prime}} \mid \psi_{\beta}\right\rangle=\delta_{\beta^{\prime} \beta}, \quad\left\langle\psi_{\beta^{\prime}}\left|H_{\beta}\right| \psi_{\beta}\right\rangle=\delta_{\beta^{\prime} \beta} \varepsilon_{\beta},
$$

where $\psi_{\gamma}$ is a pseudostate wavefunction of pair $\gamma$ and $\varepsilon_{\gamma}$ is the corresponding pseudostate energy. Then we can write

$$
\begin{aligned}
\Phi & \approx \sum_{\alpha=1}^{N_{\mathrm{H}}} F_{\alpha}\left(\boldsymbol{\rho}_{\alpha}\right) \psi_{\alpha}\left(\boldsymbol{r}_{\alpha}\right)+\sum_{\beta=1}^{N_{\mathrm{Ps}}} F_{\beta}\left(\boldsymbol{\rho}_{\beta}\right) \psi_{\beta}\left(\boldsymbol{r}_{\beta}\right) \\
& \equiv \sum_{\gamma=1}^{N_{\mathrm{H}}+N_{\mathrm{Ps}}} F_{\gamma}\left(\boldsymbol{\rho}_{\gamma}\right) \psi_{\gamma}\left(\boldsymbol{r}_{\gamma}\right)
\end{aligned}
$$

where $F_{\gamma}\left(\boldsymbol{\rho}_{\gamma}\right)$ is an unknown weight function, $\boldsymbol{r}_{\gamma}$ is the relative position of the particles in pair $\gamma, \boldsymbol{\rho}_{\gamma}$ is the position of particle $\gamma$ relative to the centre of mass (c.m.) of pair $\gamma(\gamma=\alpha, \beta)$, see Fig. 1]. For convenience, here we use the same notation not only to denote a pair and a corresponding channel, but also a quantum state in this pair and the channel. So, the indices of functions $F_{\gamma}$ and $\psi_{\gamma}$, additionally refer to a full set of quantum numbers of a state in the channel. In the case of vectors $\boldsymbol{\rho}_{\gamma}$ and $\boldsymbol{r}_{\gamma}$, the indices still refer only to a channel and a pair in the channel, respectively.

In principle, at this formal stage one could keep the continuum part only for one of the pairs in order not to double up the treatment of the three-body breakup channel. However, a symmetric expansion of the type (7), with the continuum states on both centres, was found to give fastest convergence in calculations with a manageable number of states [73, 214, 215].

Now we use the Bubnov-Galerkin principle 216] to find the coefficients $F_{\gamma}\left(\boldsymbol{\rho}_{\gamma}\right)$ so that the expansion (7) satisfies 


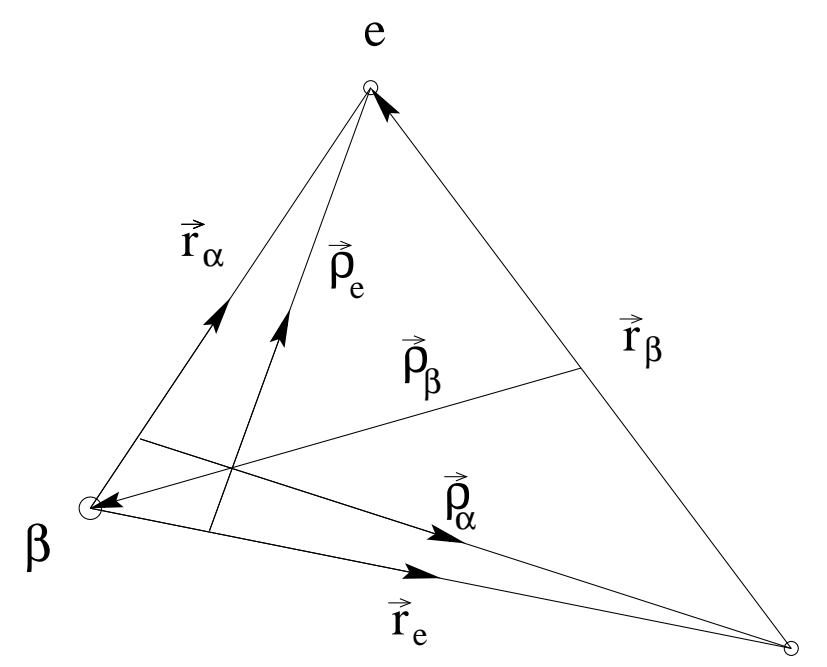

$\alpha$

FIG. 1. Jacobi coordinates for a system of three particles: positron $(\alpha)$, proton $(\beta)$, and electron $(e)$.

Eq. (2) the best possible way. Accordingly, we substitute the expansion (77) into Eq. (2) and require the result to be orthogonal to all $\left(\gamma^{\prime}=1, \ldots, N_{\mathrm{H}}+N_{\mathrm{Ps}}\right)$ basis states, i.e.

$$
\left\langle\psi_{\gamma^{\prime}}|H-E| \sum_{\gamma=1}^{N_{\mathrm{H}}+N_{\mathrm{Ps}}} F_{\gamma} \psi_{\gamma}\right\rangle_{\boldsymbol{\rho}_{\gamma^{\prime}}}=0 .
$$

In this equation subscript $\boldsymbol{\rho}_{\gamma^{\prime}}$ indicates integration over all variables except $\boldsymbol{\rho}_{\gamma^{\prime}}$. Now taking into account conditions (5) and (6) we can write Eq. (8) in the following form:

$$
\begin{aligned}
\left(E-\varepsilon_{\gamma^{\prime}}-\frac{q_{\gamma^{\prime}}^{2}}{2 M_{\gamma^{\prime}}}\right) F_{\gamma^{\prime}}\left(\boldsymbol{\rho}_{\gamma^{\prime}}\right)= & \sum_{\gamma=1}^{N_{\mathrm{H}}+N_{\mathrm{Ps}}}\left\langle\psi_{\gamma^{\prime}}\left|U_{\gamma^{\prime}}\right| \psi_{\gamma}\right\rangle \\
& \times F_{\gamma}\left(\boldsymbol{\rho}_{\gamma}\right)
\end{aligned}
$$

The potential operators $U_{\gamma^{\prime} \gamma}$ are given by

$$
\begin{aligned}
& U_{\alpha \alpha}=\bar{V}_{\alpha}, \quad U_{\beta \beta}=\bar{V}_{\beta}, \\
& U_{\alpha \beta}=U_{\beta \alpha}=H-E .
\end{aligned}
$$

The condition imposed above in Eq. (91) is a system of coupled equations for unknown expansion coefficients $F_{\gamma}\left(\boldsymbol{\rho}_{\gamma}\right)$. These functions carry information on the scattering amplitudes. We transform these integrodifferential equations for the weight functions to a set of coupled Lippmann-Schwinger integral equations for transition amplitudes $T_{\gamma^{\prime} \gamma}$.

To this end we define the Green's function in channel $\gamma$

$$
G_{\gamma}\left(q^{2}\right)=\left(E+i 0-\frac{q^{2}}{2 M_{\gamma}}-\varepsilon_{\gamma}\right)^{-1},
$$

to describe the relative motion of free particle $\gamma$ and bound pair $\gamma$ with binding energy $\epsilon_{\gamma}$. We can now write the formal solution of the differential equation (9) as

$$
\begin{aligned}
F_{\gamma^{\prime}}\left(\boldsymbol{\rho}_{\gamma^{\prime}}\right)= & \delta_{\gamma^{\prime} \gamma} e^{i \boldsymbol{q}_{\gamma} \boldsymbol{\rho}_{\gamma}}+\sum_{\gamma^{\prime \prime}=1}^{N_{\mathrm{H}}+N_{\mathrm{Ps}}} \int \frac{d \boldsymbol{q}}{(2 \pi)^{3}} e^{i \boldsymbol{q} \boldsymbol{\rho}_{\gamma^{\prime \prime}}} G_{\gamma^{\prime \prime}}\left(q^{2}\right) \\
& \times\left\langle\boldsymbol{q}\left|\left\langle\psi_{\gamma^{\prime \prime}}\left|U_{\gamma^{\prime \prime} \gamma}\right| \psi_{\gamma}\right\rangle\right| F_{\gamma}\right\rangle .
\end{aligned}
$$

The addition of positive $i 0$ defines the integration path around the singularity point at $q=q_{\gamma}=$ $\left[2 M_{\gamma}\left(E-\varepsilon_{\gamma}\right)\right]^{1 / 2}$, which is real for $E>\varepsilon_{\gamma}$, and corresponds to the outgoing wave boundary conditions. Taking Eq. (12) to the asymptotic region one can demonstrate [213] that

$$
T_{\gamma^{\prime} \gamma}\left(\boldsymbol{q}_{\gamma^{\prime}}, \boldsymbol{q}_{\gamma}\right)=\left\langle\boldsymbol{q}_{\gamma^{\prime}}\left|\left\langle\psi_{\gamma^{\prime}}\left|U_{\gamma^{\prime} \gamma}\right| \psi_{\gamma}\right\rangle\right| F_{\gamma}\right\rangle
$$

represents the matrix element for transition from channel $\gamma$ to channel $\gamma^{\prime}$, where $\boldsymbol{q}_{\gamma}$ and $\boldsymbol{q}_{\gamma^{\prime}}$ are the initial and final momenta of the incident and scattered particles. Then from (12) we get

$$
\begin{aligned}
F_{\gamma^{\prime}}\left(\boldsymbol{\rho}_{\gamma^{\prime}}\right)= & \delta_{\gamma^{\prime} \gamma} e^{i \boldsymbol{q}_{\gamma} \boldsymbol{\rho}_{\gamma}}+\sum_{\gamma^{\prime \prime}=1}^{N_{\mathrm{H}}+N_{\mathrm{Ps}}} \int \frac{d \boldsymbol{q}}{(2 \pi)^{3}} e^{i \boldsymbol{q} \boldsymbol{\rho}_{\gamma}} G_{\gamma^{\prime \prime}}\left(q^{2}\right) \\
& \times T_{\gamma^{\prime \prime} \gamma}\left(\boldsymbol{q}, \boldsymbol{q}_{\gamma}\right) .
\end{aligned}
$$

Now using Eq. (14) in Eq. (13) we get a set of coupled Lippmann-Schwinger-type equations for the transition matrix elements $\left(\gamma, \gamma^{\prime}=1, \ldots, N_{\mathrm{H}}+N_{\mathrm{Ps}}\right)$

$$
\begin{aligned}
T_{\gamma^{\prime} \gamma}\left(\boldsymbol{q}_{\gamma^{\prime}}, \boldsymbol{q}_{\gamma}\right)= & V_{\gamma^{\prime} \gamma}\left(\boldsymbol{q}_{\gamma^{\prime}}, \boldsymbol{q}_{\gamma}\right)+\sum_{\gamma^{\prime \prime}=1}^{N_{\mathrm{H}}+N_{\mathrm{Ps}}} \int \frac{d \boldsymbol{q}}{(2 \pi)^{3}} \\
& \times V_{\gamma^{\prime} \gamma^{\prime \prime}}\left(\boldsymbol{q}_{\gamma^{\prime}}, \boldsymbol{q}\right) G_{\gamma^{\prime \prime}}\left(q^{2}\right) T_{\gamma^{\prime \prime} \gamma}\left(\boldsymbol{q}, \boldsymbol{q}_{\gamma}\right) .
\end{aligned}
$$

The effective potentials are given by

$$
V_{\gamma^{\prime} \gamma}\left(\boldsymbol{q}_{\gamma^{\prime}}, \boldsymbol{q}_{\gamma}\right)=\left\langle\boldsymbol{q}_{\gamma^{\prime}}\left|\left\langle\psi_{\gamma^{\prime}}\left|U_{\gamma^{\prime} \gamma}\right| \psi_{\gamma}\right\rangle\right| \boldsymbol{q}_{\gamma}\right\rangle
$$

Thus we have a set of three-dimensional momentumspace integral equations. Eq. (15) can be solved via partial wave decomposition. The latter is an ideal method for collisions involving light projectiles like positrons [217, 218] or positronium [211, 219]. For heavy projectiles a direct three-dimensional discretization technique has been developed 220 222].

After partial wave expansion for each value of the total angular momentum $J$ one gets

$$
\begin{aligned}
\mathcal{T}_{\gamma^{\prime} \gamma}^{L^{\prime} L J}\left(q_{\gamma^{\prime}}, q_{\gamma}\right)= & \mathcal{V}_{\gamma^{\prime} \gamma}^{L^{\prime} L J}\left(q_{\gamma^{\prime}}, q_{\gamma}\right)+\sum_{\gamma^{\prime \prime}=1}^{N_{\mathrm{H}}+N_{\mathrm{Ps}}} \sum_{L^{\prime \prime}} \int \frac{d q}{\left(2 \pi^{2}\right)} \\
& \times \mathcal{V}_{\gamma^{\prime} \gamma^{\prime \prime}}^{L^{\prime} L^{\prime \prime} J}\left(q_{\gamma^{\prime}}, q\right) G_{\gamma^{\prime \prime}}\left(q^{2}\right) \mathcal{T}_{\gamma^{\prime \prime} \gamma}^{L^{\prime \prime} L J}\left(q, q_{\gamma}\right),
\end{aligned}
$$

where $L, L^{\prime}$ and $L^{\prime \prime}$ are the relative angular momenta of the fragments in channels $\gamma, \gamma^{\prime}$ and $\gamma^{\prime \prime}$, respectively. The 
effective potentials in partial waves are given by

$$
\begin{aligned}
\mathcal{V}_{\gamma^{\prime} \gamma}^{L^{\prime} L J}\left(q_{\gamma^{\prime}}, q_{\gamma}\right)= & \sum_{m^{\prime} m M^{\prime} M} \iint d \widehat{\boldsymbol{q}}_{\gamma^{\prime}} d \widehat{\boldsymbol{q}}_{\gamma} Y_{L^{\prime} M^{\prime}}^{*}\left(\widehat{\boldsymbol{q}}_{\gamma^{\prime}}\right) \\
& \times C_{L^{\prime} M^{\prime} l^{\prime} m^{\prime}}^{J K} V_{\gamma^{\prime} \gamma}\left(\boldsymbol{q}_{\gamma^{\prime}}, \boldsymbol{q}_{\gamma}\right) C_{L M l m}^{J K} \\
& \times Y_{L M}\left(\widehat{\boldsymbol{q}}_{\gamma}\right),
\end{aligned}
$$

where $C_{L M l m}^{J K}$ are the Clebsch-Gordan coefficients of vector addition, $Y_{L M}\left(\widehat{\boldsymbol{q}}_{\gamma}\right)$ is the spherical harmonics of unit vector $\widehat{\boldsymbol{q}}_{\gamma} . \quad l\left(l^{\prime}\right)$ is the angular momenta of pair $\gamma\left(\gamma^{\prime}\right)$ and $M, m, K$ are the projections of $L, l, J$, respectively, with $K=M+m=M^{\prime}+m^{\prime}$.

We introduce a K-matrix according to $\mathcal{T}=\mathcal{K} /(1+i \pi \mathcal{K})$ [68]. This reduces Eq. [17] to a set of equations for the K-matrix amplitudes

$$
\begin{aligned}
\mathcal{K}_{\gamma^{\prime} \gamma}^{L^{\prime} L J}\left(q_{\gamma^{\prime}}, q_{\gamma}\right)= & \mathcal{V}_{\gamma^{\prime} \gamma}^{L^{\prime} L J}\left(q_{\gamma^{\prime}}, q_{\gamma}\right)+\sum_{\gamma^{\prime \prime}=1}^{N_{\mathrm{H}}+N_{\mathrm{Ps}}} \sum_{L^{\prime \prime}} \mathcal{P} \int \frac{d q}{\left(2 \pi^{2}\right)} \\
& \times \frac{\mathcal{V}_{\gamma^{\prime} \gamma^{\prime \prime}}^{L^{\prime} L^{\prime \prime} J}\left(q_{\gamma^{\prime}}, q\right)}{E-q^{2} / 2 M_{\gamma^{\prime \prime}}-\epsilon_{\gamma^{\prime \prime}}} \mathcal{K}_{\gamma^{\prime \prime} \gamma}^{L^{\prime \prime} L J}\left(q, q_{\gamma}\right),
\end{aligned}
$$

where $\mathcal{P}$ indicates a principal value integral. These are the major CCC equations to be solved numerically, in the form of linear equations $A x=b$ utilising only real arithmetic [68]. Upon solution the reconstruction of the T-matrix and obtaining cross sections is a much simpler computational task.

Details of calculations of the matrix elements are given by Kadyrov and Bray 74. Here we present the final results in order to highlight the difference in complexity between the matrix elements for direct and rearrangement transitions. The effective potentials (16) for direct transitions $\alpha \rightarrow \alpha^{\prime}$ (transitions between states within the same arrangement) read

$$
\begin{aligned}
V_{\alpha^{\prime} \alpha}\left(\boldsymbol{q}_{\alpha^{\prime}}, \boldsymbol{q}_{\alpha}\right)= & \int d \boldsymbol{\rho}_{\alpha} d \boldsymbol{r}_{\alpha} e^{-i \boldsymbol{q}_{\alpha^{\prime}} \boldsymbol{\rho}_{\alpha}} \psi_{\alpha^{\prime}}^{*}\left(\boldsymbol{r}_{\alpha}\right) U_{\alpha \alpha} \\
& \times \psi_{\alpha}\left(\boldsymbol{r}_{\alpha}\right) e^{i \boldsymbol{q}_{\alpha} \boldsymbol{\rho}_{\alpha}} .
\end{aligned}
$$

In partial waves, after some algebra, we have

$$
\begin{aligned}
\mathcal{V}_{\alpha^{\prime} \alpha}^{L^{\prime} L J}\left(q_{\alpha^{\prime}}, q_{\alpha}\right)= & (4 \pi)^{2}(-1)^{J+\left(L^{\prime}+L+l^{\prime}+l\right) / 2}\left[L^{\prime} l\right] \\
& \times \sum_{\lambda}{ }^{(2)} C_{L^{\prime} 0 \lambda 0}^{L 0} C_{l 0 \lambda 0}^{l^{\prime} 0}\left\{\begin{array}{ccc}
L^{\prime} & \lambda & L \\
l & J & l^{\prime}
\end{array}\right\} \\
& \times I_{\alpha^{\prime} \alpha}^{\lambda}\left(q_{\alpha^{\prime}}, q_{\alpha}\right)
\end{aligned}
$$

where $[l]=\sqrt{2 l+1},[l L]$ is a shorthand for $[l][L]$, and the braces denote the $6 j$-symbol. In the sum over $\lambda$ only terms preserving the parity of $l^{\prime}+l$ survive, leading to increments of 2 . The radial integral is defined as

$$
\begin{aligned}
I_{\alpha^{\prime} \alpha}^{\lambda}\left(q_{\alpha^{\prime}}, q_{\alpha}\right)= & \int_{0}^{\infty} d \rho_{\alpha} \rho_{\alpha}^{2} j_{L^{\prime}}\left(q_{\alpha^{\prime}} \rho_{\alpha}\right) j_{L}\left(q_{\alpha} \rho_{\alpha}\right) \\
& \times \int_{0}^{\infty} d r_{\alpha} r_{\alpha}^{2} R_{n^{\prime} l^{\prime}}\left(r_{\alpha}\right) \mathcal{U}_{\alpha \alpha}^{\lambda}\left(\rho_{\alpha}, r_{\alpha}\right) R_{n l}\left(r_{\alpha}\right),
\end{aligned}
$$

where $R_{n l}(r)$ are the radial parts of the pseudostate wavefunctions $\psi$ and

$$
\mathcal{U}_{\alpha \alpha}^{\lambda}\left(\rho_{\alpha}, r_{\alpha}\right)= \begin{cases}\frac{\delta_{\lambda 0}}{\rho_{\alpha}}-\frac{\rho_{\alpha}^{\lambda}}{r_{\alpha}^{\lambda+1}} & \text { if } \rho_{\alpha}<r_{\alpha} \\ \frac{\delta_{\lambda 0}}{\rho_{\alpha}}-\frac{r_{\alpha}^{\lambda}}{\rho_{\alpha}^{\lambda+1}} & \text { otherwise. }\end{cases}
$$

Matrix elements for $\beta \rightarrow \beta^{\prime}$ transitions are defined as

$$
\begin{aligned}
V_{\beta^{\prime} \beta}\left(\boldsymbol{q}_{\beta^{\prime}}, \boldsymbol{q}_{\beta}\right)= & \int d \boldsymbol{\rho}_{\beta} d \boldsymbol{r}_{\beta} e^{-i \boldsymbol{q}_{\beta^{\prime}} \boldsymbol{\rho}_{\beta}} \psi_{\beta^{\prime}}^{*}\left(\boldsymbol{r}_{\beta}\right) U_{\beta \beta} \\
& \times \psi_{\beta}\left(\boldsymbol{r}_{\beta}\right) e^{i \boldsymbol{q}_{\beta} \boldsymbol{\rho}_{\beta}} .
\end{aligned}
$$

The corresponding partial-wave amplitudes $\mathcal{V}_{\beta^{\prime}}^{L^{\prime} L}\left(q_{\beta^{\prime}}, q_{\beta}\right)$ have the same form as expression (21) but with $\mathcal{U}_{\beta \beta}^{\lambda}\left(\rho_{\beta}, r_{\beta}\right)$ in Eq. (22) for $I_{\beta^{\prime} \beta}^{\lambda}\left(q_{\beta^{\prime}}, q_{\beta}\right)$ defined as

$$
\mathcal{U}_{\beta \beta}^{\lambda}\left(\rho_{\beta}, r_{\beta}\right)=\left(1-(-1)^{\lambda}\right) \begin{cases}\frac{2^{\lambda+1} \rho_{\beta}^{\lambda}}{r_{\beta}^{\lambda+1}} & \text { if } \rho_{\beta}<r_{\beta} / 2 \\ \frac{r_{\beta}^{\lambda}}{2^{\lambda} \rho_{\beta}^{\lambda+1}} & \text { otherwise. }\end{cases}
$$

In the case of rearrangement $\alpha \rightarrow \beta$ transitions Eq. (16) for the effective potentials reads as

$$
\begin{aligned}
V_{\beta \alpha}\left(\boldsymbol{q}_{\beta}, \boldsymbol{q}_{\alpha}\right)= & \int d \boldsymbol{\rho}_{\beta} d \boldsymbol{r}_{\beta} e^{-i \boldsymbol{q}_{\beta} \boldsymbol{\rho}_{\beta}} \psi_{\beta}^{*}\left(\boldsymbol{r}_{\beta}\right)(H-E) \\
& \times \psi_{\alpha}\left(\boldsymbol{r}_{\alpha}\right) e^{i \boldsymbol{q}_{\alpha} \boldsymbol{\rho}_{\alpha}} .
\end{aligned}
$$

Calculation of the matrix elements for the rearrangement transitions is significantly more complicated. Using the Gaussian representation for the wavefunctions and the interaction potentials Hewitt et al. [61] calculated the Ps-formation matrix elements for transition amplitudes between arbitrary $\mathrm{H}$ and Ps states. Mitroy [63] suggested a straightforward way of calculating the matrix elements without using additional expansions. Following Mitroy 63], Kadyrov and Bray 74] further reduced the results for the case of the Laguerre-type pseudostates analytically calculating integrals for momentum-space pseudostate wavefunctions and formfactors. The final result for the matrix elements in the rearrangement channels is written as

$$
\begin{aligned}
& \mathcal{V}_{\beta \alpha}^{L^{\prime} L}\left(q_{\beta}, q_{\alpha}\right)=\left[l^{\prime} l L^{\prime} L\right]\left[l^{\prime} ! l !\right](-1)^{J+L^{\prime}} \sum_{l_{1}^{\prime}} \frac{\left[l_{1}^{\prime} l_{2}^{\prime}\right]}{\left[l_{1}^{\prime} ! l_{2}^{\prime} !\right]} 2^{-l_{1}^{\prime}-1} \\
& \times \sum_{l_{1}} \frac{\left[l_{1} l_{2}\right]}{\left[l_{1} ! l_{2} !\right]} q_{\beta}^{l_{1}^{\prime}+l_{1}} \sum_{l_{1}^{\prime \prime}}^{(2)} C_{l_{1} 0 l_{1}^{\prime} 0}^{l_{1}^{\prime \prime} 0} \\
& \times \sum_{l_{2}^{\prime \prime}}^{(2)} C_{l_{2} 0 l_{2}^{\prime} 0}^{l_{2}^{\prime \prime} 0} \sum_{\lambda}^{(2)}[\lambda]^{2} C_{L_{1}^{\prime} 0 \lambda 0}^{l_{1}^{\prime \prime} 0} C_{L 0 \lambda 0}^{l_{2}^{\prime \prime} 0} \\
& \times\left\{\begin{array}{llllllll}
l_{1} & & & l & & J & & l^{\prime} \\
& l_{2} & & L & & L^{\prime} & & l_{1}^{\prime} \\
l_{2}^{\prime} & & l_{2}^{\prime \prime} & & \lambda & & l_{1}^{\prime \prime} &
\end{array}\right\} \\
& \times I_{\beta \alpha}^{\lambda}\left(q_{\beta}, q_{\alpha}\right),
\end{aligned}
$$


where $[l !]=\sqrt{(2 l+1) !}, l_{1}^{\prime}+l_{2}^{\prime}=l^{\prime}$ and $l_{1}+l_{2}=l$, the braces denote the $12 j$-symbol of the first kind [223],

$$
\begin{aligned}
I_{\beta \alpha}^{\lambda}\left(q_{\beta}, q_{\alpha}\right)= & q_{\alpha}^{l_{2}^{\prime}+l_{2}} \mathcal{F}_{\lambda}^{(I)}\left(q_{\beta}, q_{\alpha}\right)+\frac{1}{\pi q_{\alpha}} \int_{0}^{\infty} d q q^{l_{2}^{\prime}+l_{2}+1} \\
& \times Q_{L}\left(\frac{q^{2}+q_{\alpha}^{2}}{2 q q_{\alpha}}\right) \mathcal{F}_{\lambda}^{(I I)}\left(q_{\beta}, q\right)
\end{aligned}
$$

and where $Q_{L}$ is a Legendre function of the second kind. For Legendre polynomial $P_{\lambda}$ and $z=\widehat{\boldsymbol{q}}_{\beta} \cdot \widehat{\boldsymbol{q}}_{\alpha}$,

$$
\mathcal{F}_{\lambda}^{(I, I I)}\left(q_{\beta}, q_{\alpha}\right)=\int_{-1}^{1} d z F^{(I, I I)}\left(\boldsymbol{q}_{\beta}, \boldsymbol{q}_{\alpha}\right) P_{\lambda}(z)
$$

with

$$
\begin{aligned}
F^{(I)}\left(\boldsymbol{q}_{\beta}, \boldsymbol{q}_{\alpha}\right)= & \left(\frac{q_{\alpha}^{2}}{2}+\frac{p_{\alpha}^{2}}{2}-E\right) \frac{\widetilde{R}_{n^{\prime} l^{\prime}}^{*}\left(p_{\beta}\right) \widetilde{R}_{n l}\left(p_{\alpha}\right)}{p_{\beta}^{l^{\prime}} p_{\alpha}^{l}} \\
& +\frac{\widetilde{R}_{n^{\prime} l^{\prime}}^{*}\left(p_{\beta}\right) \widetilde{u}_{n l}\left(p_{\alpha}\right)}{p_{\beta}^{l^{\prime}} p_{\alpha}^{l}}+\frac{\widetilde{u}_{n^{\prime} l^{\prime}}^{*}\left(p_{\beta}\right) \widetilde{R}_{n l}\left(p_{\alpha}\right)}{p_{\beta}^{l^{\prime}} p_{\alpha}^{l}}
\end{aligned}
$$

and

$$
F^{(I I)}\left(\boldsymbol{q}_{\beta}, \boldsymbol{q}\right)=\frac{\widetilde{R}_{n^{\prime} l^{\prime}}^{*}\left(p_{\beta}^{\prime}\right) \widetilde{R}_{n l}\left(p_{\alpha}^{\prime}\right)}{p_{\beta}^{\prime l^{\prime}} p_{\alpha}^{\prime l}},
$$

where $\widetilde{R}_{n l}(p)$ and $\widetilde{u}_{n l}(p)$ are the pseudostate wavefunctions and pseudostate formfactors in momentum space, respectively. Here $\boldsymbol{p}_{\gamma}$ is the relative momentum of the particles of pair $\gamma$ :

$$
\boldsymbol{p}_{\beta}=\boldsymbol{q}_{\beta} / 2-\boldsymbol{q}_{\alpha} \text { and } \boldsymbol{p}_{\alpha}=\boldsymbol{q}_{\beta}-\boldsymbol{q}_{\alpha} .
$$

In addition, $\boldsymbol{p}_{\alpha}^{\prime}$ and $\boldsymbol{p}_{\beta}^{\prime}$ are the relative momenta of the particles of the corresponding pairs immediately before and after the rearrangement:

$$
\boldsymbol{p}_{\beta}^{\prime}=\boldsymbol{q}_{\beta} / 2-\boldsymbol{q} \text { and } \boldsymbol{p}_{\alpha}^{\prime}=\boldsymbol{q}_{\beta}-\boldsymbol{q},
$$

where $\boldsymbol{q}$ is the relative momentum of the fragments in channel $e$.

As mentioned before, 2 sets of pseudostates are obtained by diagonalising the $\mathrm{H}$ and $\mathrm{Ps}$ Hamiltonians. The radial parts of pseudostates are taken as

$$
R_{n l}(r)=\sum_{k=1}^{N} B_{n k}^{l} \xi_{k l}(r)
$$

where pseudostate basis $\xi_{k l}(r)$ is constructed from the orthogonal Laguerre functions

$$
\xi_{k l}(r)=N_{k l}(2 r / a)^{l+1} e^{-r / a} L_{k-1}^{2 l+2}(2 r / a),
$$

with

$$
N_{k l}=\left[\frac{2(k-1) !}{a(2 l+1+k) !}\right]^{1 / 2} .
$$

Here $L_{k-1}^{2 l+2}(2 r / a)$ are the associated Laguerre polynomials. Expansion coefficients $B_{n k}^{l}$ are found by diagonalizing the two-particle Hamiltonian of the relevant pair.
As to the choice of the exponential fall-off parameter, practice shows that the highest rate of convergence is achieved when $a_{\alpha}$ and $a_{\beta}$ are chosen to best reproduce the ground state energy of hydrogen and positronium, respectively, for a given size of the bases $N_{\mathrm{H}}$ and $N_{\mathrm{Ps}}$. The choice of $a_{\alpha}=1$ and $a_{\beta}=0.5$ fulfils this requirement. Associated pseudostate wavefunctions and form factors in momentum space used in Eq. (30) and Eq. (31) have been calculated in analytic form.

The set of equations (19) is solved using standard quadrature rules. The singular kernel is discretised using a Gauss quadrature. A subtraction technique is used to handle moving singularities. Very recently Bray et al. 224, 225] have proposed a new approach to solving Eq. (19), which handles the Green's function analytically, see Sec. IVB

Cross section from initial state $\alpha$ to final state $\alpha^{\prime}$ (or $\beta$ ) is calculated as a cross section for excitation of state $\alpha^{\prime}(\beta)$. The total cross section is obtained as a sum of all partial cross sections for each state included in the close-coupling expansion, or utilizing the unitarity of the close-coupling formalism by applying the optical theorem. We calculate the total cross section in both ways (which should give the same result) to check that the optical theorem is satisfied. The total ionization cross section is calculated as a sum of the integrated cross sections for positive energy states (of both atom and Ps). The total Ps-formation cross section is defined as a sum of the cross sections for electron capture into Ps bound states.

\section{B. Helium}

A distinct feature of the $\mathrm{CCC}$ formalism is that the resulting set of coupled equations (19) is essentially independent of the choice of the target, and is similar to that given for hydrogen. Therefore, the basic formalism of the two-centre CCC method described above can be applied to positron collisions with helium. However, in this case spins play an important role. There are two target electrons that can form positronium. The electrons can be in spin-singlet or spin-triplet states. Depending on the spin projections of the electron and the positron that form positronium, the latter can be formed in para ( $\mathrm{p}-\mathrm{Ps}$ ) or ortho (o-Ps) states. The target spin and Ps spin should couple into the total spin that stays unchanged during the scattering process. However, separation of the spatial part of the total scattering wavefunction from the spin part is a non-trivial task.

Here it is more convenient to adopt a coordinate system, where $\boldsymbol{r}_{0}, \boldsymbol{r}_{1}$, and $\boldsymbol{r}_{2}$ denote the positions of the positron, electrons 1 and 2, respectively (see Fig. 2), relative to the helium nucleus, while $\boldsymbol{R}=\left(\boldsymbol{r}_{0}+\boldsymbol{r}_{1}\right) / 2$ is the position of the Ps centre relative to the He nucleus and $\boldsymbol{\rho}=\boldsymbol{r}_{0}-\boldsymbol{r}_{1}$ is the relative coordinate of the positron and electron 1. Fig. 2 depicts one of the two possible systems of coordinates $\left(\boldsymbol{r}_{0}, \boldsymbol{r}_{1}, \boldsymbol{r}_{2}\right)$ and $\left(\boldsymbol{R}, \boldsymbol{\rho}, \boldsymbol{r}_{2}\right)$. There 
are two sets of Jacobi coordinates corresponding to the two electrons that can form positronium. When necessary we will refer to them explicitly as $\left(\boldsymbol{R}_{1}, \boldsymbol{\rho}_{1}, \boldsymbol{r}_{2}\right)$ and $\left(\boldsymbol{R}_{2}, \boldsymbol{\rho}_{2}, \boldsymbol{r}_{1}\right)$. Fig. 2 shows the one where Ps is formed by electron 1 .

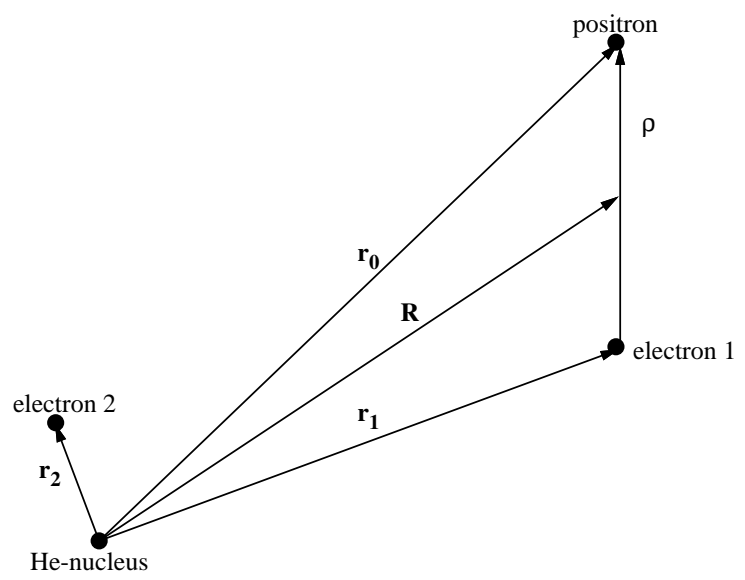

FIG. 2. Coordinate systems for positron-helium scattering, where we assume that positronium is formed with electron 1 . In this case the generic $R$ and $\rho$ become $R_{1}$ and $\rho_{1}$. For the case where positronium is formed with electron 2 we use $R_{2}$ and $\rho_{2}$.

The Schrödinger equation for the total scattering wavefunction $\Psi$ of the positron-helium system with the total energy $E$ is written as

$$
(H-E) \Psi^{s S M}\left(\boldsymbol{x}_{0}, \boldsymbol{x}_{1}, \boldsymbol{x}_{2}\right)=0,
$$

where $\boldsymbol{x}_{0}, \boldsymbol{x}_{1}$ and $\boldsymbol{x}_{2}$ are the full sets of coordinates of the particles including their spin. When spin-orbit interactions are neglected it should be possible (at this stage this is merely an assumption; we will see later if this is indeed the case) to separate the radial and spin parts according to

$$
\Psi^{s S M}\left(\boldsymbol{x}_{0}, \boldsymbol{x}_{1}, \boldsymbol{x}_{2}\right)=\Phi^{s S}\left(\boldsymbol{r}_{0}, \boldsymbol{r}_{1}, \boldsymbol{r}_{2}\right) \chi_{s S M}(0,(1,2)),
$$

where $\Phi^{s S}\left(\boldsymbol{r}_{0}, \boldsymbol{r}_{1}, \boldsymbol{r}_{2}\right)$ is the wavefunction that depends on spatial coordinates and $\chi_{s S M}(0,(1,2))$ is the spin state of the two electrons (with combined two-particle spin $s$ ) and the positron with the total spin $S$ and its projection $M$. We expand the total wavefunction $\Psi^{s S M}$ in terms of states of all asymptotic channels and require the result to be antisymmetric against permutation of the two electrons:

$$
\begin{aligned}
\Psi^{s S M} \approx & \frac{1}{\sqrt{2}}\left(1-P_{12}\right)\left[\sum_{\alpha=1}^{N_{\mathrm{He}}} F_{\alpha}^{(s)}\left(\boldsymbol{r}_{0}\right) \psi_{\alpha}\left(\boldsymbol{r}_{1}, \boldsymbol{r}_{2}\right)\right. \\
& \times \chi_{s S M}(0,(1,2)) \\
& +\sum_{s^{\prime}} \sum_{\beta=1}^{N_{\mathrm{Ps}}} G_{\beta}^{\left(s^{\prime}\right)}\left(\boldsymbol{R}_{1}\right) \psi_{\beta}\left(\boldsymbol{\rho}_{1}\right) \phi_{1 s}\left(\boldsymbol{r}_{2}\right) \\
& \left.\times \chi_{s^{\prime} S M}((0,1), 2)\right]
\end{aligned}
$$

where $\psi_{\alpha}$ are the helium wavefunctions and $\psi_{\beta}$ are the Ps ones. Expansion coefficients $F_{\alpha}^{(s)}$ and $G_{\beta}^{\left(s^{\prime}\right)}$ depend on the spins $s$ and $s^{\prime}$ of He and Ps, respectively. The $N_{\mathrm{He}}$ and $N_{\mathrm{Ps}}$ are the numbers of the He and Ps pseudostates. The second term represents Ps formation by both electrons and the sum over $s^{\prime}$ corresponds to Ps formation in para $\left(s^{\prime}=0\right)$ and ortho $\left(s^{\prime}=1\right)$ states. The wavefunction $\phi_{1 s}$ describes the residual ion of $\mathrm{He}^{+}$. The latter is considered to be in the $1 s$ state only. The operator $\frac{1}{\sqrt{2}}\left(1-P_{12}\right)$, where $P_{12}$ is a permutation operator that interchanges the electrons 1 and 2 , insures that the wavefunction is anti-symmetric. The spin wavefunctions are written as 125]

$$
\chi_{s S M}(0,(1,2))=\sum_{\mu_{0}, \mu} C_{\frac{1}{2} \mu_{0} s \mu}^{S M} \chi_{\frac{1}{2} \mu_{0}}(0) \chi_{s \mu}(1,2)
$$

for the $\mathrm{e}^{+}-$He channel, and

$$
\chi_{s^{\prime} S M}((0,1), 2)=\sum_{\mu_{2}, \mu^{\prime}} C_{\frac{1}{2} \mu_{2} s^{\prime} \mu^{\prime}} \chi_{s^{\prime} \mu^{\prime}}(0,1) \chi_{\frac{1}{2} \mu_{2}}(2)
$$

for the $\mathrm{Ps}-\mathrm{He}^{+}$channel.

As the spin-orbit interactions are neglected, the initial spin of the target $s$, the total spin $S$ and consequently the total spin function must be conserved. Therefore the spatial part in Eq. (38) can be written as

$$
\Phi^{s S}\left(\boldsymbol{r}_{0}, \boldsymbol{r}_{1}, \boldsymbol{r}_{2}\right)=\left\langle\chi_{s S M}(0,(1,2)) \mid \Psi^{s S M}\right\rangle .
$$

Spin algebra to find the right-hand side of Eq. (42) was perform by Utamuratov et al. [126]. They showed that the spin wavefunctions satisfy the following relations

$$
\begin{gathered}
\chi_{s S M}(0,(2,1))=(-1)^{s+1} \chi_{s S M}(0,(1,2)), \\
\chi_{s^{\prime} S M}((0,1), 2)=\sum_{s^{\prime \prime}} c_{s^{\prime} s^{\prime \prime} S} \chi_{s^{\prime \prime} S M}(0,(1,2))
\end{gathered}
$$

and

$$
\chi_{s^{\prime} S M}((0,2), 1)=\sum_{s^{\prime \prime}}(-1)^{s^{\prime \prime}+1} c_{s^{\prime} s^{\prime \prime} S} \chi_{s^{\prime \prime} S M}(0,(1,2)),
$$

where the overlap coefficients are given by the $6 \mathrm{j}$-symbol

$$
c_{s^{\prime} s^{\prime \prime} S}=(-1)^{S-\frac{1}{2}}\left[s^{\prime} s^{\prime \prime}\right]\left\{\begin{array}{ccc}
1 / 2 & 1 / 2 & s^{\prime} \\
S & 1 / 2 & s^{\prime \prime}
\end{array}\right\} .
$$

Taking into account Eqs. (43)-(45) and writing the sum over $s^{\prime}$ explicitly (the Ps spin $s^{\prime}$ can be 0 or 1 ) we have

$$
\begin{aligned}
\Phi^{s S} \approx & \sum_{\alpha=1}^{N_{\mathrm{He}}} F_{\alpha}^{(s)}\left(\boldsymbol{r}_{0}\right) \psi_{\alpha}^{s}\left(\boldsymbol{r}_{1}, \boldsymbol{r}_{2}\right) \\
& +\frac{1}{\sqrt{2}} \sum_{\beta=1}^{N_{\mathrm{Ps}}}\left\{\tilde{G}_{\beta}^{(s S)}\left(\boldsymbol{R}_{1}\right) \psi_{\beta}\left(\boldsymbol{\rho}_{1}\right) \phi_{1 s}\left(\boldsymbol{r}_{2}\right)\right. \\
& \left.+(-1)^{s} \tilde{G}_{\beta}^{(s S)}\left(\boldsymbol{R}_{2}\right) \psi_{\beta}\left(\boldsymbol{\rho}_{2}\right) \phi_{1 s}\left(\boldsymbol{r}_{1}\right)\right\}
\end{aligned}
$$


where

$$
\psi_{\alpha}^{s}\left(\boldsymbol{r}_{1}, \boldsymbol{r}_{2}\right)=\frac{1}{\sqrt{2}}\left\{\psi_{\alpha}\left(\boldsymbol{r}_{1}, \boldsymbol{r}_{2}\right)+(-1)^{s} \psi_{\alpha}\left(\boldsymbol{r}_{2}, \boldsymbol{r}_{1}\right)\right\}
$$

is the antisymmetrized helium wavefunction and

$$
\tilde{G}_{\beta}^{(s S)}(\boldsymbol{R})=c_{0 s S} G_{\beta}^{(0)}(\boldsymbol{R})+c_{1 s S} G_{\beta}^{(1)}(\boldsymbol{R}) .
$$

We emphasize that the superscript $s$ of $\tilde{G}_{\beta}$ is the spin of the target, while that of $G_{\beta}$ is the spin of the formed positronium. Note that

$$
G_{\beta}^{(1)}=\sqrt{3} G_{\beta}^{(0)}
$$

This relationship is often assumed and used in the literature, however, its origin remained unclear. It was rigorously derived for the first time by Utamuratov et al. [126].

The anti-symmetrised wavefunction $\psi_{\alpha}^{s}\left(\boldsymbol{r}_{1}, \boldsymbol{r}_{2}\right)$ for helium is built using the configuration interaction (CI) approach [226]. Two types of approximations are used: a frozen core (FC), where one of the electrons is described by the $\mathrm{He}^{+} 1$ s orbital and a multi-core $(\mathrm{MC})$, where the core electron is described by any number of orbitals as necessary to yield as accurate He wave functions as desired. However, as mentioned earlier the residual ion in the $\mathrm{Ps}_{\mathrm{S}} \mathrm{He}^{+}$channels is considered to be always in the $1 s$-state.

Eqs. (39, [47) show that the spin part of the total wavefunction is factorized and, therefore, can be removed from the equations. Consequently, for a given total spin $S$ and target spin $s$, the Schrödinger equation (37) transforms to an equation for the spatial part of the total wavefunction $\Phi^{s S}$ :

$$
(H-E) \Phi^{s S}\left(\boldsymbol{r}_{0}, \boldsymbol{r}_{1}, \boldsymbol{r}_{2}\right)=0,
$$

where $\Phi^{s S}$ is given by Eq. (47).

Direct scattering and Ps-formation matrix elements have been given by Utamuratov et al. 125]. Due to the two-centre expansion the system of equations (19) is highly ill-conditioned. The ill-conditioning makes it impossible to use arbitrarily high basis sizes, and requires the matrix elements to be calculated to high precision.

\section{Alkali metals}

We model an alkali atom as a system with one active electron above a frozen Hartree-Fock core [227]. Accordingly, the positron-alkali collision is treated as a threebody system of the incoming positron, the active (outer shell) electron and an inert core ion. The interaction between the active electron and the inert core $V_{\alpha}$ is calculated as a static part of the Hartree-Fock potential $V_{\text {st }}$ supplemented by an exchange potential $V_{\text {ex }}$ between the active and the core electrons. Thus the interactions in this model system are the electron-ion $V_{\alpha}$, positron-ion
$V_{e}$, and electron-positron $V_{\beta}$ potentials defined as follows

$$
\begin{aligned}
& V_{\alpha}(r)=V_{\mathrm{st}}(r)+V_{\mathrm{ex}}(r)+V_{\mathrm{pol}}(r), \\
& V_{e}(r)=-V_{\mathrm{st}}(r)+V_{\mathrm{pol}}(r), \\
& V_{\beta}(r)=-1 / r .
\end{aligned}
$$

The static term is calculated by

$$
V_{\mathrm{st}}(r)=-\frac{Z}{r}+2 \sum_{\psi_{j} \in C} \int d^{3} r^{\prime} \frac{\left|\psi_{j}\left(\boldsymbol{r}^{\prime}\right)\right|^{2}}{\left|\boldsymbol{r}-\boldsymbol{r}^{\prime}\right|},
$$

where $Z$ is the charge of the target nucleus and $\psi_{j}$ are the states of the ion core $C$ generated by performing the self-consistent-field Hartree-Fock calculations [227]. The summation in Eq. (55) is done for all core states. The equivalent local-exchange approximation [228 230] is used to take into account the exchange between the active electron and core electrons:

$$
\begin{aligned}
V_{\mathrm{ex}}\left(r, E_{\mathrm{ex}}\right)= & \frac{1}{2}\left[\left(E_{\mathrm{ex}}-V_{\mathrm{st}}(r)\right)\right. \\
& \left.-\sqrt{\left(E_{\mathrm{ex}}-V_{\mathrm{st}}(r)\right)^{2}+\rho(r)}\right],
\end{aligned}
$$

where

$$
\rho(r)=\sum_{\psi_{j} \in C} \int \mathrm{d} \hat{\boldsymbol{r}}\left|\psi_{j}(\boldsymbol{r})\right|^{2}
$$

and $E_{\text {ex }}$ is an adjustment parameter. The core polarization potential $V_{\text {pol }}$ in Eq. (52) is

$$
V_{\mathrm{pol}}(r)=-\frac{\alpha_{d}}{2 r_{0}^{4}} \cdot \frac{1-\exp \left[-x^{6}\right]}{x^{4}}
$$

where $x=r / r_{0}, \alpha_{d}$ is the dipole polarizability and $r_{0}$ are adjustable parameters to fit some physical quantities (e.g., energies of the valence electron).

\section{Magnesium and inert gases}

$\mathrm{Mg}$ is modelled as a He-like system with two active electrons above a frozen Hartree-Fock core [155, 231, 232]. The interaction between an active electron and the frozen Hartree-Fock core is calculated as a sum of the static part of the Hartree-Fock potential and an exchange potential between an active and the core electrons as described in the previous subsection. Details of the two-centre CCC method for positron-Mg collisions are given in [156].

Wavefunctions for the inert gases of $\mathrm{Ne}, \mathrm{Ar}, \mathrm{Kr}$, and $\mathrm{Xe}$ are described by a model of six p-electrons above a frozen Hartree-Fock core. Discrete and continuum target states are obtained by allowing one-electron excitations from the p-shell in the following way. Taking Ne as an example, self-consistent Hartree-Fock calculations are performed for the $\mathrm{Ne}^{+}$ion, resulting in the $1 \mathrm{~s}, 2 \mathrm{~s}, 2 \mathrm{p}$ orbitals. The $1 \mathrm{~s}$ and $2 \mathrm{~s}$ orbitals are treated as the inert core orbitals, while the $2 \mathrm{p}$ Hartree-Fock orbital is used as the frozen-core orbital to form the target states. A set of 
Laguerre functions is used to diagonalize the quasi-oneelectron Hamiltonian of the $\mathrm{Ne}^{5+}$ ion, utilising the nonlocal Hartree-Fock potential constructed from the inert core orbitals. The resulting $2 \mathrm{p}$ orbital differs substantially from the Hartree-Fock $2 \mathrm{p}$ orbital. A one-electron basis suitable for the description of a neutral Ne atom is built by replacing the $2 \mathrm{p}$ orbital from diagonalisation with the Hartree-Fock one, orthogonalized by the GramSchmidt procedure. The six-electron target states are described via the configuration-interaction (CI) expansion. The set of configurations is built by angular momentum coupling of the wavefunction of $2 \mathrm{p}^{5}$ electrons and the Laguerre-based one-electron functions. The coefficients of the CI expansion are obtained by diagonalization of the target Hamiltonian. The target orbital angular momentum $l$, spin $s$, and parity $\pi$ are conserved quantum numbers and diagonalization of the target Hamiltonian is performed separately for each target symmetry $\{l, s, \pi\}$. Full details of the single centre CCC calculations for noble gas atoms are given in Ref. [163]. A two-centre approach to inert gases has not yet been attempted.

\section{E. Molecular hydrogen}

Positron- $\mathrm{H}_{2}$ scattering can be treated somewhat similar to the helium case. We consider $\mathrm{H}_{2}$ within the BornOppenheimer approximation where the two protons are considered to be at a fixed internuclear distance denoted as $d$. Expansion for the total scattering wavefunction (after separation of the spin part) is similar to Eq. (47) for He. However, the wavefunctions for the target in the first term and the residual ion in the Ps-formation channel depend on $d$. The residual ion of $\mathrm{H}_{2}^{+}$, with same internuclear distance $d$, is considered to be only in its ground state. We only use a few Ps eigenstates so as to take advantage of their analytical form. The target states are obtained by diagonalizing the $\mathrm{H}_{2}$ Hamiltonian in a set of antisymmetrized two-electron configurations, built from Laguerre one-electron orbitals, for each target symmetry characterized by the projection of orbital angular momentum, parity and spin. To calculate $\mathrm{H}_{2}$ states, we use the fixed-nuclei approximation. Calculations are performed at the ground-state equilibrium internuclei distance taken to be $d=1.4 \mathrm{a}_{0}$. When $d$ is set to 0 one should obtain the He results. We used this test for both structure and scattering calculations. Details of $\mathrm{H}_{2}$ structure calculations can be found in [196 198].

The derivation of the rearrangement matrix elements are somewhat more difficult than for He because of their dependence on the nuclear separation and target orientation. Another difference is that partial wave expansion is done over the total angular momentum projection $K$. It is convenient to choose the z-axis to be along the $\boldsymbol{d}$ vector (body-frame). Then it is possible to transform the obtained results with this choice of z-axis to any given orientation of the molecule. To facilitate the calculations only the spherical part of the nuclear potential is considered when calculating the rearrangement matrix elements:

$$
V_{\mathrm{p}}\left(\boldsymbol{r}_{0}, \boldsymbol{d}\right)=\frac{1}{\left|\boldsymbol{r}_{0}-\boldsymbol{d} / 2\right|}+\frac{1}{\left|\boldsymbol{r}_{0}+\boldsymbol{d} / 2\right|} \approx \frac{2}{r_{>}},
$$

where $r_{>}=\max \left\{r_{0}, d / 2\right\}$. Then the momentum space representation of the above positron-nucleus potential can be shown to be

$$
\bar{V}_{\mathrm{p}}(p)=\frac{4 \pi^{2} \sin (d p)}{d p^{3}}
$$

With these one further follows the procedure used for positron-He calculations 125.

For positron collisions with the ground state of $\mathrm{H}_{2}$ only states with zero total spin are required and so $S=1 / 2$. $T$-matrix elements are used for obtaining body-frame scattering amplitudes. These are then rotated by Euler angles to transform them to lab-frame scattering amplitudes. Orientationally-independent cross sections are calculated by averaging over all rotations of the molecule 233]. An orientationally averaged analytic Born subtraction method [233] is employed for $\mathrm{H}_{2}$ direct transition channels. This helped reduce the number of partial waves requiring explicit calculation.

\section{RECENT APPLICATIONS OF THE CCC THEORY TO POSITRON SCATTERING}

\section{A. Internal consistency}

Fundamentally, in order for a theory to be useful it needs to be predictive. In the close-coupling approach to electron/positron/proton scattering on relatively simple targets, where the structure is readily obtained, there are two computational problems that need to be overcome. The first, is that for a given set of states used to expand the total wavefunction the resulting equations need to be solved to an acceptable numerical precision. The second is to systematically increase the size of the expansion and demonstrate that the final results converge to a unique answer that is independent of the choice of the expansion, so long as it is sufficiently large. Only once this is achieved can we be in a position to claim that the results are the true solution of the underlying Schrödinger equation and hence predictive of what should happen in the experiment.

In the case of electron scattering there are only onecentre expansions because electrons do not form bound states with the electrons of the target. Electron exchange is handled within the potential matrix elements all based on the coordinate origin at the nucleus. Accordingly, establishing convergence in just the one-centre approach is all that needs to be done, though historically this was a major challenge [37, 68]. Though convergence was shown to be to a result that disagreed with experiment [68], subsequent experiments showed excellent agreement with the CCC theory [234, 235], which lead to reanalysis of 
the original data 236] yielding good agreement with the $\mathrm{CCC}$ theory and new experiments. A similar situation occurred in the case of double photoionisation of helium 237, 238], which in effect is electron scattering on the singly charged helium ion [239, 240].

For positron and proton scattering the issue of convergence is even more interesting due to the capacity of the projectile to form a bound state with a target electron. This leads to a second natural centre in the problem which also requires treatment to convergence. For positron scattering on atomic hydrogen the Ps-formation threshold is at $6.8 \mathrm{eV}$, while the ionisation threshold is $13.6 \mathrm{eV}$. However, Ps formation is also a form of ionisation of the target except that the electron is captured to a bound state of the projectile. Any expansion of the Ps centre using a complete basis will result in negative- and positive-energy states, with the latter corresponding to three-body breakup. However, expansion of the atomic centre will also generate independent positive-energy states corresponding to three-body breakup. Hence, expansions using a complete basis on each centre, will yield independent, non-orthogonal states corresponding to the same physical three-body breakup process. While this may appear to be a fundamental problem, in practice it is an interesting strength of the method which allows to check internal consistency of the results. By this we mean that the same results must be obtained from a variety of calculations utilising independent one- and two-centre expansions as detailed below.

We begin by considering two extremes: the first attempts to obtain convergence using only the atomic centre, while the second attempts convergence using two complete expansion on both centres. Will either converge, and if they do, will the convergence be to the same result?

In Fig. 3 typical energies arising in two-centre calculations are given. We see a similar spread of negative- and positive-energy states on both the $\mathrm{H}$ and the Ps centres. A single centre expansion based on the atomic centre would not have any Ps states included in the calculation.

The results of the two types of calculations may be readily summarised by Fig. 4 . On the left we have onecentre cross sections $\sigma_{f i}^{(1)}$, where $i, f=1, \ldots, N_{\mathrm{H}}^{(1)}$. Taking the initial state to be the ground state of $\mathrm{H}(i=1)$ then $\sigma_{11}^{(1)}$ is the elastic scattering cross section, and $\sigma_{f 1}^{(1)}$ corresponds to excitation whenever $\varepsilon_{f 1}<0$ and ionisation whenever $\varepsilon_{f 1}>0$. The elastic and excitation cross sections need to converge with increasing $N_{\mathrm{H}}^{(1)}$ individually. However, the ionisation cross sections converge as a sum, yielding the total ionisation cross section $\sigma_{\text {ion }}^{(1)}=\sum_{f} \sigma_{f 1}^{(1)}$ for $\varepsilon_{f}^{(1)}>0$.

Convergence of the one-centre $\mathrm{CCC}$ calculations, where there are no Ps states, has been studied extensively [70, 121, 122]. Briefly, at energies below the Psformation threshold the important contribution of virtual Ps formation is adequately treated via the positiveenergy atomic states of large angular momentum $l_{\max } \approx$

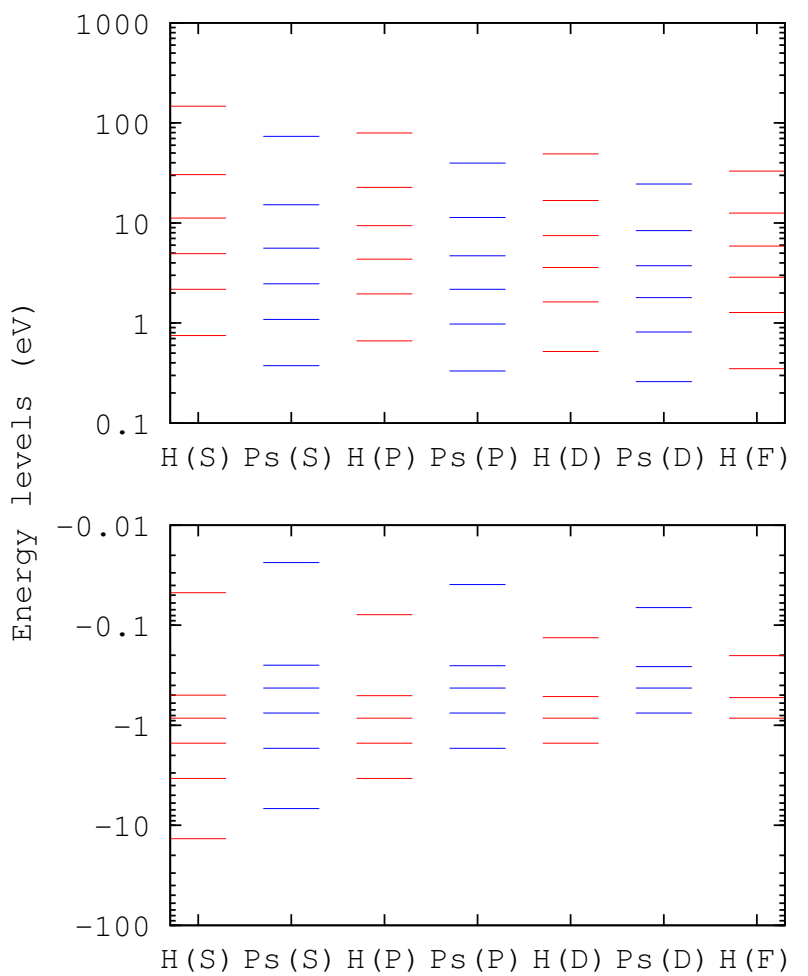

FIG. 3. Energies $\varepsilon_{n l}^{(\mathrm{H})}$ and $\varepsilon_{n l}^{(\mathrm{Ps})}$, arising upon diagonalisation of the respective Hamiltonians, in the $\mathrm{CCC}\left(12_{3}, 12_{2}\right)$ positronhydrogen calculations. Here $12-l$ states were obtained for each $l$ with $l_{\max }=3$ for $\mathrm{H}$ states and $l_{\max }=2$ for Ps states, see Kadyrov et al. 211].

10. This allows for convergence of elastic scattering cross section to the correct value. At energies above the ionisation threshold, the positive-energy atomic pseudostates take into account both breakup and Ps-formation cross section in a collective way yielding the correct electronloss and excitation cross sections. However, in the extended Ore gap region between the Ps-formation and ionisation thresholds no convergence is possible due to all positive-energy pseudostates being closed.

Convergence in two-centre calculations is potentially problematic at all energies due to two independent treatments of the breakup processes. In practice this manifests itself as an ill-conditioned system which requires high-precision matrix elements and limits the size of the calculations i.e. $N_{\mathrm{H}}^{(2)}$ and $N_{\mathrm{Ps}}^{(2)}$ are typically substantially smaller than $N_{\mathrm{H}}^{(1)}$. It is for this reason that we have drawn the one-center matrix to be substantially larger in Fig. [4 Furthermore, the H-Ps matrix elements take at least an order of magnitude longer to calculate due to the non-separable nature of the radial integrals [74]. Accordingly, even with much smaller number of states (with smaller $\left.l_{\max }\right)$ the two-centre calculations take considerably longer to complete. Nevertheless convergence is obtained for individual transitions involving discrete states, 


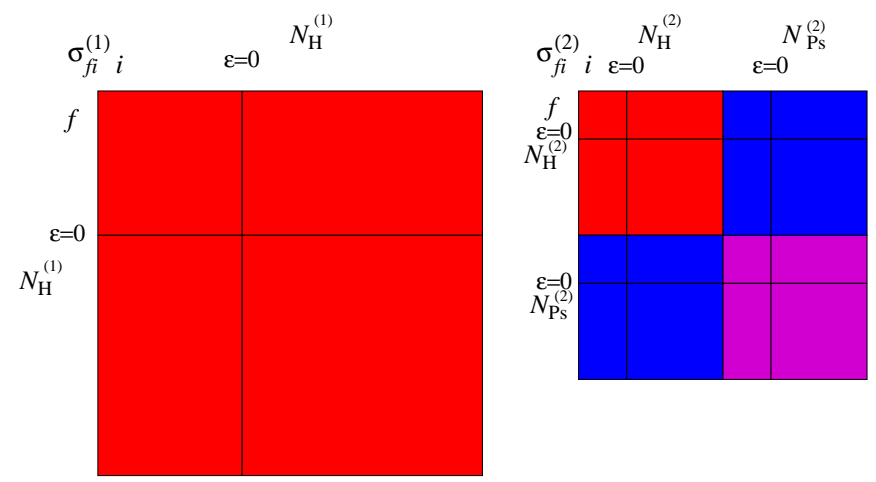

FIG. 4. Matrix overview of the cross sections $\sigma_{f i}$ arising from one-centre (left) and two-centre (right) CCC positronhydrogen calculations. $N_{\mathrm{H}}^{(1)}$ is the number of $\mathrm{H}$ states in the one-centre calculation which has no explicit Ps states. $N_{\mathrm{H}}^{(2)}$ is the number of $\mathrm{H}$ states in the two-centre calculation with $N_{\mathrm{Ps}}^{(2)}$ explicit Ps states. First presented by Bray et al. [241].

explicit Ps formation, and explicit breakup [218, 241]. Internal consistency is satisfied if at energies outside the extended Ore gap, for discrete $\left(\varepsilon_{f}^{\mathrm{A}}, \varepsilon_{i}^{\mathrm{A}}<0\right)$ atomic transitions the two approaches independently converge such that

$$
\sigma_{f i}^{(2)}=\sigma_{f i}^{(1)}
$$

Furthermore, at energies above the breakup threshold

$$
\begin{aligned}
\sigma_{\mathrm{eloss}}^{(2)} & =\sigma_{\mathrm{Ps}}^{(2)}+\sigma_{\mathrm{brk}}^{(2)} \\
& =\sigma_{\mathrm{ion}}^{(1)},
\end{aligned}
$$

where for some initial state $i$

$$
\begin{aligned}
\sigma_{\mathrm{Ps}}^{(2)} & =\sum_{f: \varepsilon_{f}^{\mathrm{Ps}}<0} \sigma_{f i}^{(2)}, \\
\sigma_{\mathrm{brk}}^{(2)} & =\sum_{f: \varepsilon_{f}^{\mathrm{Ps}}>0} \sigma_{f i}^{(2)}+\sum_{f: \varepsilon_{f}^{\mathrm{A}}>0} \sigma_{f i}^{(2)}, \\
\sigma_{\text {ion }}^{(1)} & =\sum_{f: \varepsilon_{f}^{\mathrm{A}}>0} \sigma_{f i}^{(1)} .
\end{aligned}
$$

In the extended Ore gap only the two-centre calculations are able to yield convergent results.

The great strength of the internal-consistency check is that it is available (outside the extended Ore gap) for every partial wave of the total orbital angular momentum. Checking that Eq. (62) is satisfied for every partial wave provides confidence in the overall results of the two completely independent calculations, which will typically have very different convergence properties with increasing $N$ and $l$. Due to the unitarity of the close-coupling theory agreement for Eq. (62) suggests agreement for other channels, and so Eq. (61) will also hold.

In Fig. 5 we give the example of an internal-consistency check, presented by Bray et al. 241]. We see that outside the extended Ore gap the two calculations are generally
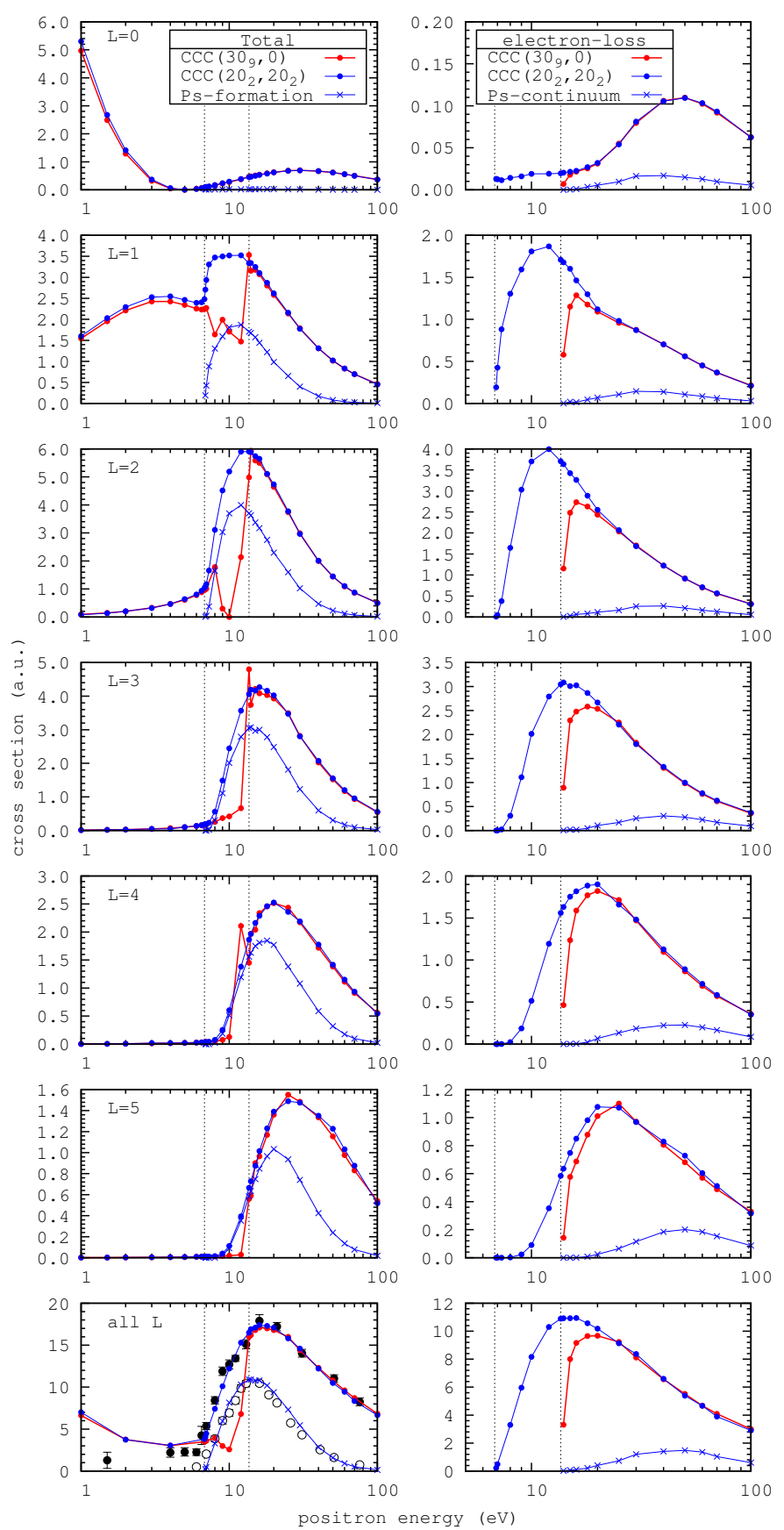

FIG. 5. Total and electron-loss (Ps formation plus breakup) cross sections for positron scattering on atomic hydrogen for specified partial waves $L$ obtained using the one- and twocentre CCC calculations, see text. The indicated points corresponding to the energies at which the calculations were performed are connected with straight lines to guide the eye. The vertical lines are the Ps-formation and breakup thresholds, spanning the extended Ore gap. The experimental data in the bottom left panel are due to Zhou et al. [178]. First presented by Bray et al. 241]. 
in very good agreement. One systematic exception is at just above the ionisation threshold. Here the breakup cross section is almost zero, but the Ps-formation cross section is near its maximum. Even with $N_{\mathrm{H}}^{(1)}=30$ the one-centre calculation does not have enough pseudostates of energy just a little above zero which would be necessary to reproduce the what should be step-function behaviour in one-centre CCC calculations. Due to explicit Ps-formation in the two-centre calculations there are no such problems here or within the extended Ore gap. Having checked the individual partial waves, and summing over all to convergence, excellent agreement is found with experiment. Having performed the internal consistency checks we remain confident in the theoretical results even if there is potentially a discrepancy with experiment at the lowest energy measured.

\section{B. Atomic hydrogen}

Establishing convergence in the cross sections with a systematically increasing close-coupling expansion places a severe test on the scattering formalism. This is as relevant to positron scattering as it is for electron scattering. Pseudoresonances must disappear with increased size of the calculations, and uncertainty in the final results can be established via the convergence study.

One of the earliest successes of the two-centre CCC method for positron-hydrogen $S$-wave scattering was to show how the Higgins-Burke pseudoresonance [62] disappeared utilising a $(\bar{N}, \bar{M})$ basis of only $s$-states on each centre [3]. The cross sections for all reaction channels were shown to converge to a few $\%$ with a $(\overline{16}, \overline{16})$ basis of $s$-states. Interestingly, the symmetric treatment of both centres was particularly efficient in terms of reaching convergence and eliminating pseudoresonances, with no double-counting problems.

The question of convergence in the case of the full positron-hydrogen scattering problem was investigated by Kadyrov and Bray 74. Setting $M=N$, states of higher angular momentum were increased systematically. The same level of convergence as in the $S$-wave model case was achieved with the $(\overline{34}, \overline{34})$ basis made of ten $s$-, nine $p$-, eight $d$ - and seven $f$-states for each centre, for scattering on the ground state. The largest calculations performed had a total of 68 states, 34 each of $\mathrm{H}$ and Ps states. The convergence was checked for the total and other main cross sections corresponding to transitions to negative-energy states. Reasonably smooth cross sections were obtained for all bases with $l$-convergence being rather rapid. For the three cases considered $f$-states contribute only marginally.

Figs. 6, 8 show the CCC results in comparison with other calculations and experimental data of Detroit 178] and London [76] groups. The CCC results agree well with experiment. So do $\mathrm{CC}(\overline{30}, 3)$ calculations of Kernoghan et al. 71] and $\mathrm{CC}(\overline{28}, 3)$ calculations of Mitroy [72]. Note that in these calculations the $n^{-3}$ scaling rule was used

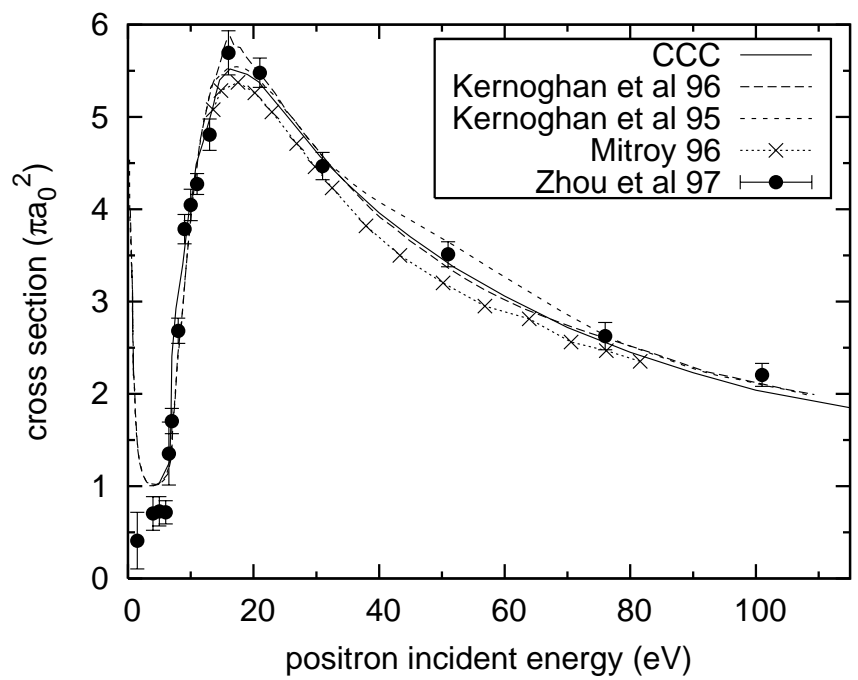

FIG. 6. Total cross section for $e^{+}+\mathrm{H}$ scattering. The experimental results are due to Zhou et al. [178]. The CCC result is from 74 . The other theoretical results are due to Kernoghan et al. [65, 71] and Mitroy [2].

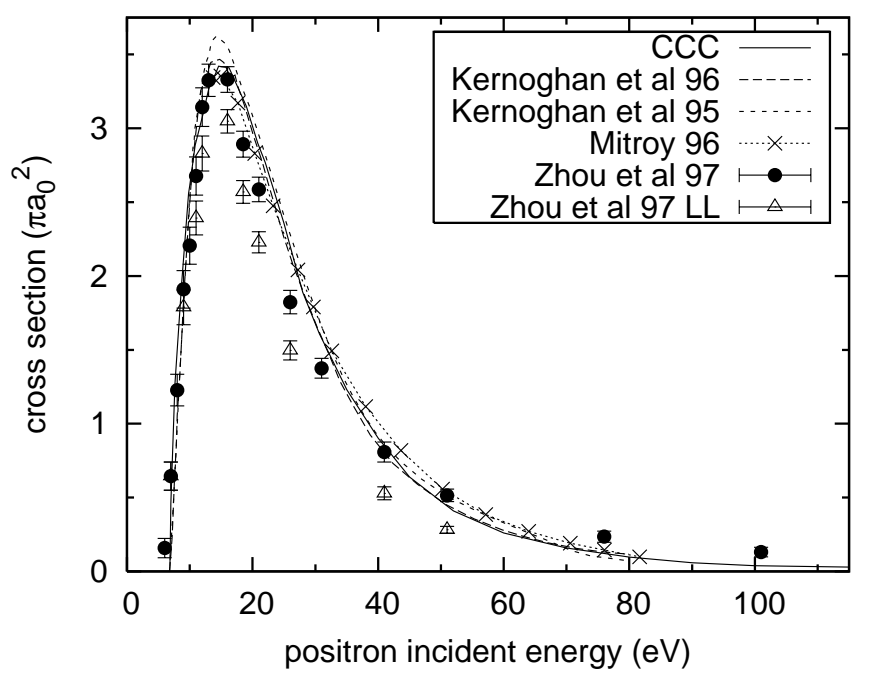

FIG. 7. Total Ps-formation cross section for $e^{+}+\mathrm{H}$ scattering. The experimental results are due to Zhou et al. 178] (LL indicated lower limit). The CCC result is from 74 . The other theoretical results are due to Kernoghan et al. 65, 71. and Mitroy [2].

to estimate the total Ps formation. Also, an energyaveraging procedure was used to smooth the $\mathrm{CC}(\overline{9}, \overline{9})$ calculations of Kernoghan et al. [65]. In the CCC method convergence is established without such procedures being used.

For the breakup cross section the CCC results have two comparable contributions, one from the excitation 


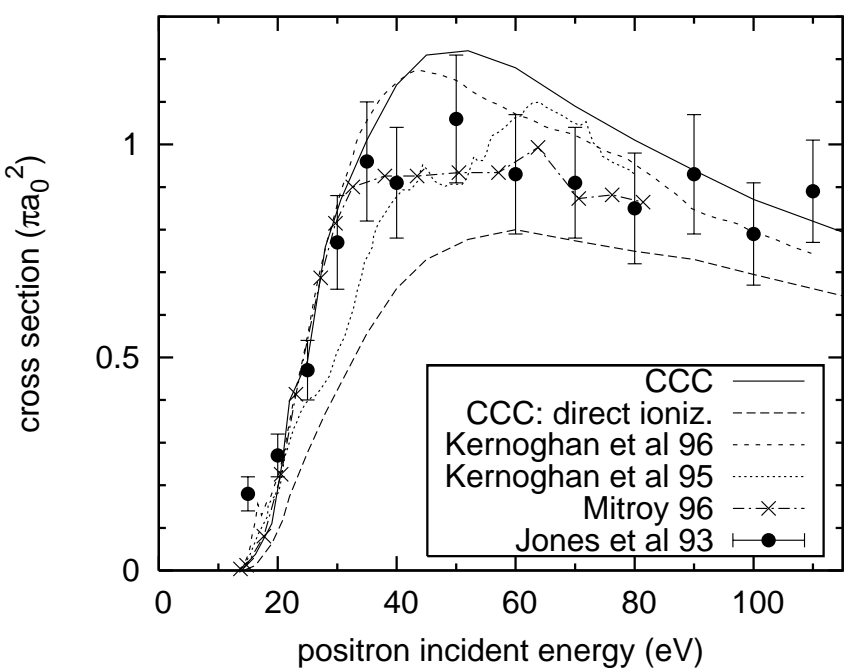

FIG. 8. Total breakup cross section for $e^{+}+\mathrm{H}$ scattering. The experimental results are due to Jones et al. 76]. The CCC result is from [4]. The other theoretical results are due to Kernoghan et al. [65, 71] and Mitroy [2].

of the positive-energy $\mathrm{H}$ pseudostates (shown in Fig. 8 as direct ionization), and the other from excitation of positive-energy Ps pseudostates. This was also noted by Kernoghan et al. 65] using the $\operatorname{CC}(\overline{9}, \overline{9})$ calculations. By contrast, in $\operatorname{CC}(\bar{N}, M)$-type calculations the contribution to breakup comes only from from direct ionisation. At the maximum of the cross section the separately converged indirect contribution to the breakup cross section is approximately a third of the total. However, the $\mathrm{CC}(\overline{30}, 3)$ cross section of Kernoghan et al. 71] is only a marginally smaller, indicating that absence of Ps positive-energy states is absorbed by the positive-energy $\mathrm{H}$ states.

Fig. 9 shows the CCC results of Fig. 8 , but against excess (total) energy to emphasize the lower energies. The full $\mathrm{CCC}(\mathrm{H}+\mathrm{Ps})$ results with breakup cross sections coming from both the $\mathrm{H}$ and Ps centres are contrasted with those just from $\mathrm{H}$ and twice $\mathrm{H}$ (labeled as $\mathrm{CCC}(\mathrm{H}+\mathrm{H})$ ). We see that below about $20 \mathrm{eV}$ excess energy the $\mathrm{CCC}(\mathrm{H}+\mathrm{Ps})$ and $\mathrm{CCC}(\mathrm{H}+\mathrm{H})$ curves are much the same, indicating that the $\mathrm{Ps}$ and $\mathrm{H}$ contributions to breakup converge to each other as the threshold is approached.

Utilising the CCC method Kadyrov et al. 217] reported calculations of positron-hydrogen scattering near the breakup threshold in order to examine the threshold law. The results are given in Fig. 10. The Wannierlike threshold law, derived by Ihra et al. [242], is in good agreement with the CCC results below $1 \mathrm{eV}$ excess energy. This law was derived for the $L=0$ partial wave, and Rost and Heller [243] showed the same energy-dependence holds in all partial waves. As for the

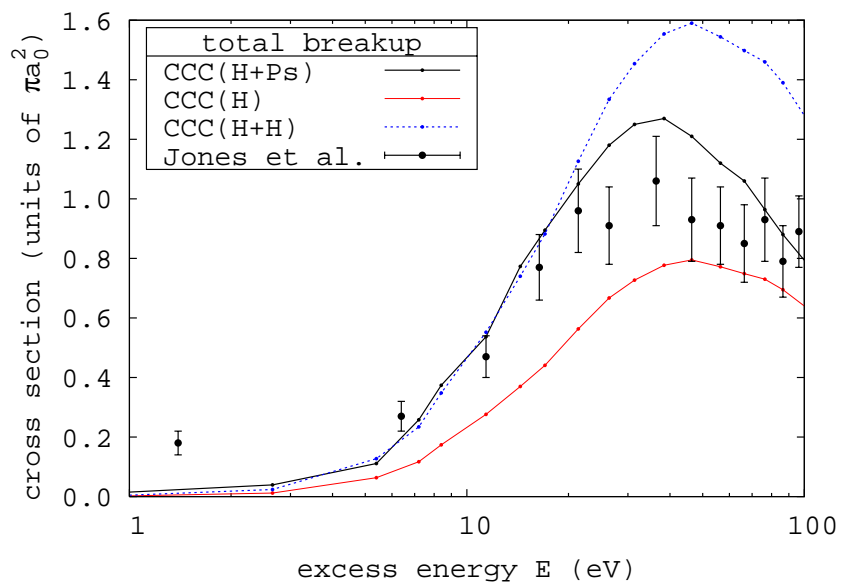

FIG. 9. Total $e^{+}-\mathrm{H}$ breakup cross section as a function of excess energy calculated using the two-center CCC method, first presented by Kadyrov et al. [217]. The argument to the CCC label indicates which center's positive-energy states were used, see text. The experiment is due to Jones et al. 76].

full problem the contributions from both centres to the breakup cross section converge to each other with decreasing excess energy, without any over-completeness problems.

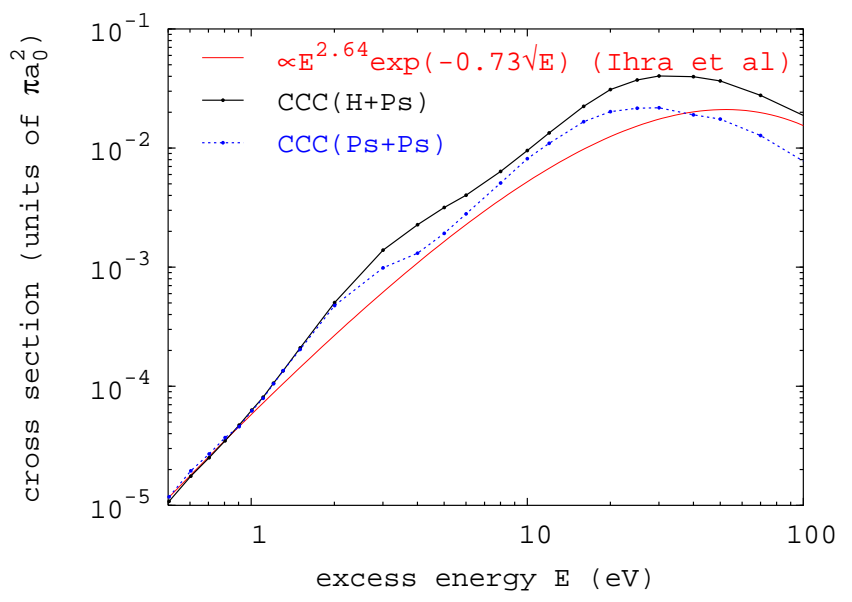

FIG. 10. Total $e^{+}-\mathrm{H} S$-wave model breakup cross section as a function of excess energy calculated using the two-center CCC method, from Kadyrov et al. [217]. As in Fig. 9, the argument to the CCC label indicates which center's positiveenergy states were used. The Wannier-like threshold law is due to Ihra et al. 242]

\section{Helium}

Helium in its ground state is the most frequently used target in experimental studies of positron-atom scattering. First measurements on positron-helium scattering 
were carried out by Canter et al. 91] in 1972. Since then many other experimental studies have been conducted 84, 86, 101, 112 114, 244, 245. Further developments of positron beams in terms of energy resolution and beam intensities have recently motivated more experimental studies [20, 110, 246 250]. In general, the results from the experiments agree well with each other. A complete theoretical approach from low to high energies had been lacking until the development of the CCC method for the problem by Utamuratov et al. [125].

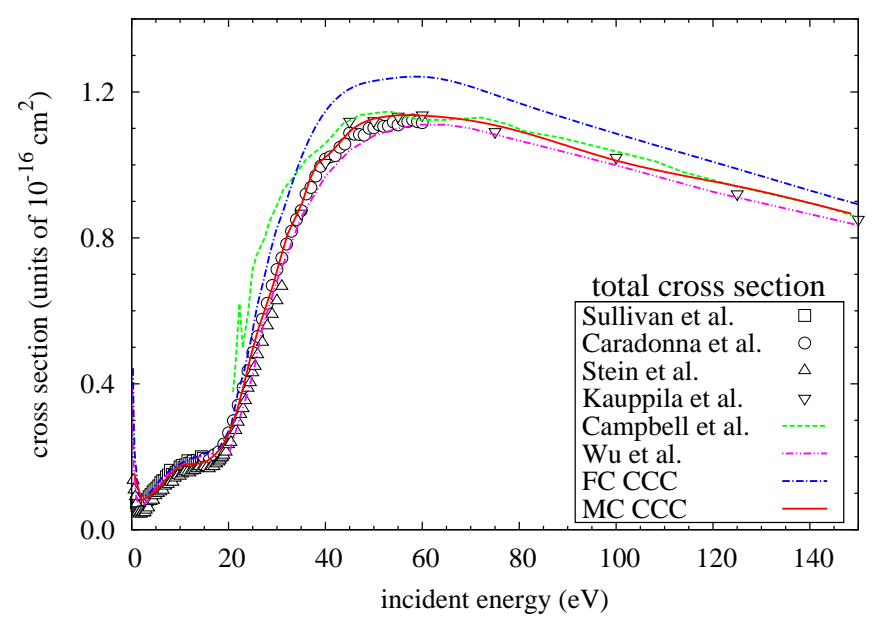

FIG. 11. Total positron-helium scattering cross section. Experimental data are due to Stein et al. [101], Sullivan et al. [20], Kauppila et al. [112], and Caradonna et al. [250]. The calculations are due to Campbell et al. [111] and Wu et al. [122]. The FC CCC and MC CCC results are from Utamuratov et al. [125.

A vast amount of experimental data is available for integrated cross sections for positron scattering from the helium ground state. The total CCC-calculated cross section is shown in Fig. 11 in comparison with experimental data and other calculations. Considerable discussion on the topic has been presented by Utamuratov et al. [125]. It suffices to say that so long as an accurate ground state is used, obtained from a multi-core (MC) treatment, agreement with experiment is outstanding across all energies. We expect the frozen-core (FC) treatment of helium to result in systematically larger excitation and ionization cross sections because it understimate the ionization potential by around $0.84 \mathrm{eV}$. Generally, a larger ionisation potential leads to a smaller cross section. In the CCC method we are not free to replace calculated energies with those from experiment, as this leads to numerical inconsistency. Consequently, there is no way to avoid the extra complexity associated with the MC calculations if high accuracy is required.

The total Ps-formation, breakup and electron-loss cross sections are given in Fig. 12(a), (b) and (c), respectively. Beginning with Ps formation, given the minor

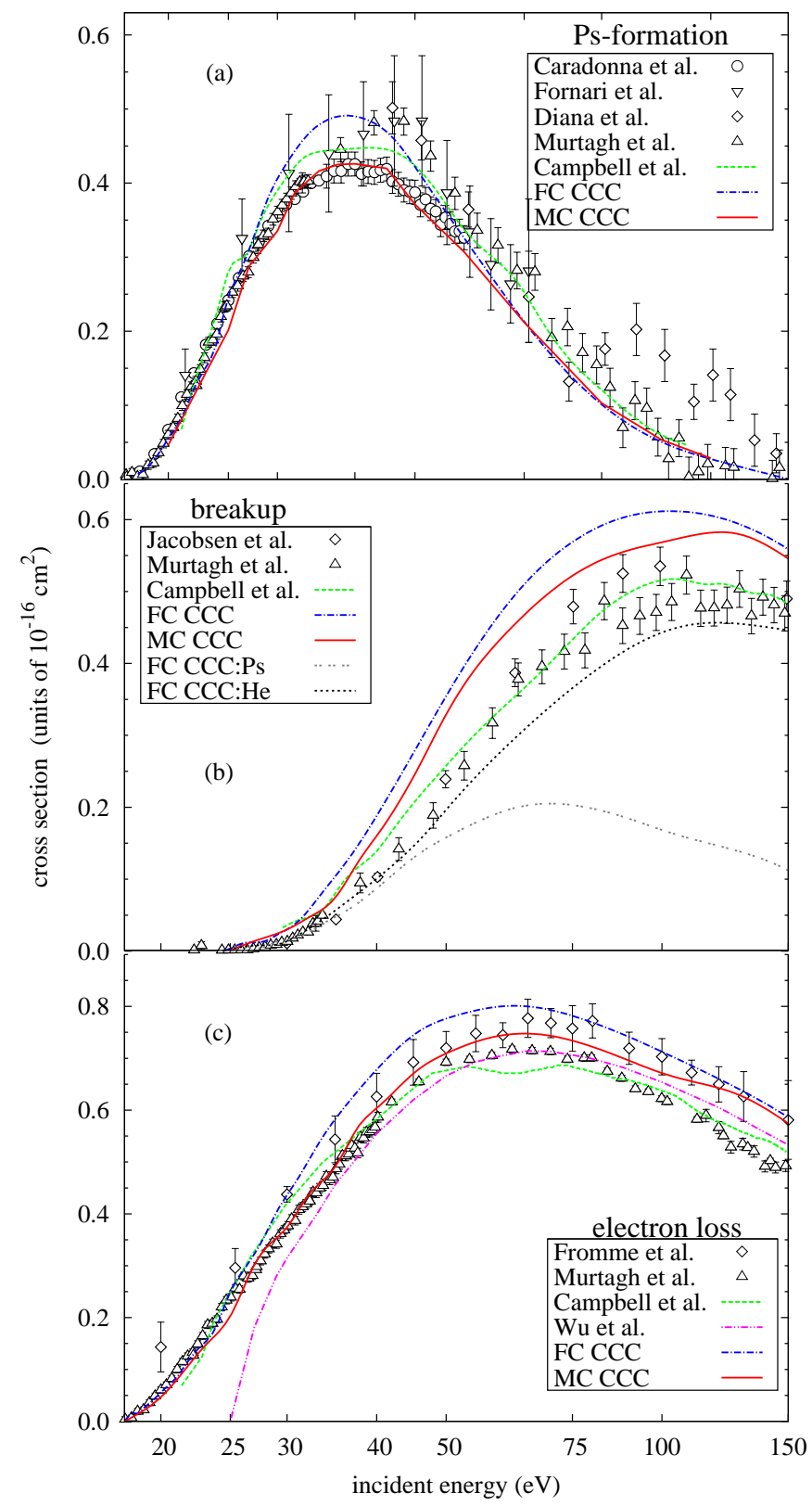

FIG. 12. Positron-helium (a) Ps-formation, (b) breakup, and (c) electron-loss (sum of the other two) cross sections. The measurements are due to Caradonna et al. 250], Fornari et al. 113], Diana et al. [114], Murtagh et al. 246], Fromme et al. 84] and Knudsen et al. [85]. The calculations are due to Campbell et al. [111], Wu et al. [122]. The FC CCC and MC CCC results are from 125].

variation in the measurements agreement between the various theories and experiment is satisfactory. However, turning our attention to the breakup cross section we see that (MC) CCC appears to be substantially higher than experiment. Yet when these cross sections are summed to form the electron-loss cross section, the agreement with the experiment of Fromme et al. 84] which measured this 
directly, is good. Given the complexity of the problem and the experimental uncertainties, the agreement with experiment is very satisfying.

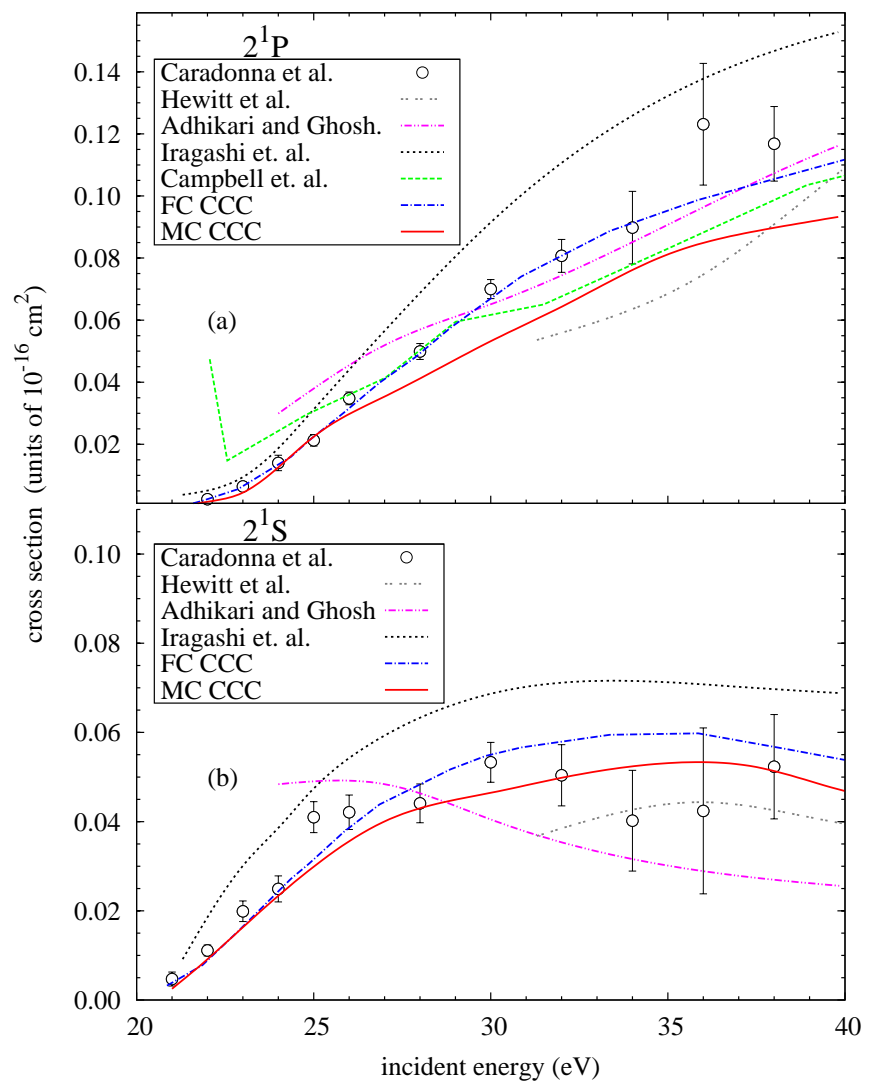

FIG. 13. Integrated cross sections for (a) $\mathrm{He}\left(2{ }^{1} \mathrm{~S}\right)$ and (b) $\mathrm{He}\left(2^{1} \mathrm{P}\right)$ excitations by positron impact. Experiment is due to Caradonna et al. 250]. The FC CCC and MC CCC results are from [125], other calculations are due to Hewitt et al. [116], Adhikari and Ghosh [251], Igarashi et al. [120] and Campbell et al. [11].

The cross sections of $2{ }^{1} \mathrm{~S}$ and $2{ }^{1} \mathrm{P}$ excitation of helium are presented in Fig. 13a and b, respectively. The MC $\mathrm{CCC}$ result for $2^{1} \mathrm{~S}$ is in good agreement with the data of Caradonna et al. [250] while the $2^{1} \mathrm{P}$ result is somewhat lower than experiment. The fact that the FC CCC $2^{1} \mathrm{P}$ results agree better with the experimental data is fortuitous. Other available theories show some systematic difficulties for these relatively small cross sections.

The cross sections for the rather exotic Ps formation in the $2 s$ and $2 p$ excited states are presented in Fig. 14(a) and (b) respectively. The cross sections are particularly small. Nevertheless, agreement with the sole available experiment of Murtagh et al. [247] is remarkable.

Thus we have seen that there is good agreement between theory and experiment for the integrated cross sections for positron scattering on helium and hydrogen in their ground states. In both cases the ionisation thresholds are well above $6.8 \mathrm{eV}$, and so a one-centre calculation is applicable at low energies where elastic scattering is the

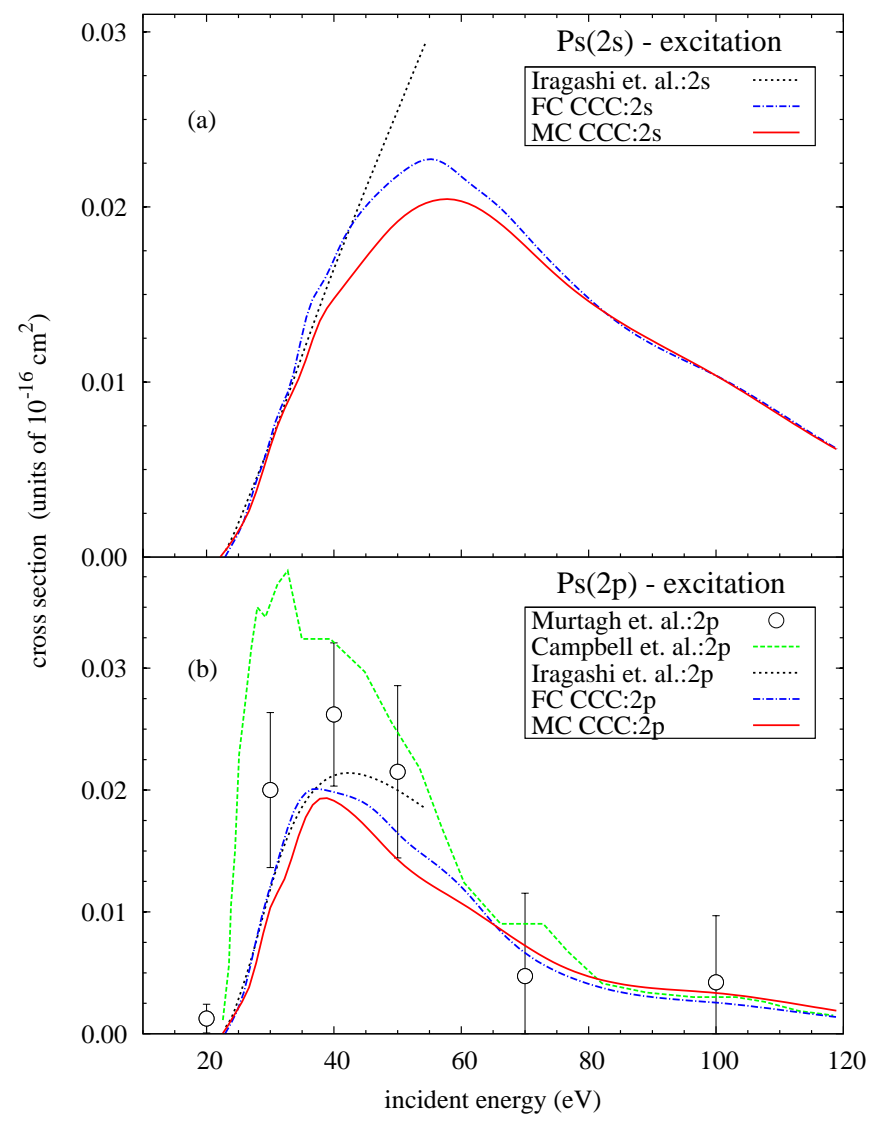

FIG. 14. Integrated cross sections for Ps formation in the (a) $2 s$ and (b) $2 p$ states. Experimental data for $\operatorname{Ps}(2 p)$ are due to Murtagh et al. 247]. The FC CCC and MC CCC results are from [125] and other calculations are due to Campbell et al. [11] and Igarashi et al. [120].

only open channel. Though experimentally challenging positron scattering on either $\mathrm{H}$ or $\mathrm{He}$ metastable excited states results in Ps formation being an open channel at all energies. Taking the example of positron scattering from the $2^{3} \mathrm{~S}$ metastable state of helium the Ps threshold is negative $(-2.06 \mathrm{eV})$. This collision system has been extensively studied by Utamuratov et al. [126]. As far as we are aware, no experimental studies have been conducted for positron scattering on metastable states of helium. Given the experimental work on electron scattering from metastable helium [252], in a group that also has a positron beam, we are hopeful that in the future there might be experimental data available for such systems. As there are still unresolved discrepancies between theory and experiment regarding electron scattering from metastable states of He [253 255], using positrons instead of electrons may assist with their resolution. 


\section{Alkali metals}

Just like for $\mathrm{H}$ and $\mathrm{He}$ in metastable states, for positron collisions with alkali-metal atoms in their ground state, both elastic and Ps(1s) formation channels are open even at zero positron energy Accordingly, we require twocentre expansions even at the lowest incident energies. Lugovskoy et al. [136] conducted two-centre calculations with different basis sets to achieve results that are independent of the Laguerre exponential fall-off parameter $\lambda_{l}$, and convergent with the Laguerre basis size $N_{l}=N_{0}-l$ for target orbital angular momentum $l \leq l_{\max }$.

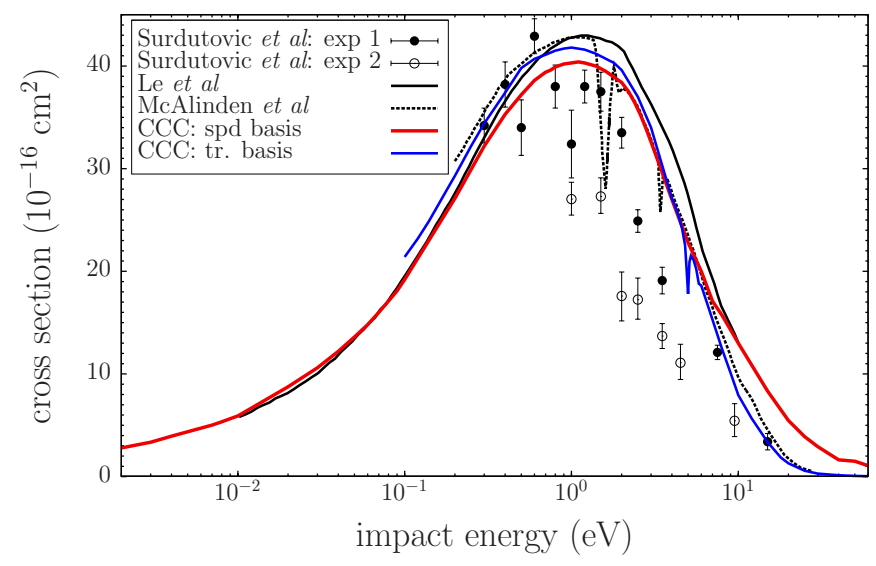

FIG. 15. Total positronium-formation cross section for $\mathrm{e}^{+}-$ Li along with the experimental points by Surdutovich et al. [137] and theoretical calculations by McAlinden et al. [130], Le et al. [135]. The truncated basis (CCC tr) calculation is an attempt to reproduce the states used by McAlinden et al. [130], see Lugovskoy et al. [136] for details.

Figure 15 shows the positronium-formation cross section. Agreement between the various calculations is quite good, while comparison with the experimental data of Surdutovich et al. 137] is rather mixed. The key feature is that the Ps-formation cross section diminishes with decreasing energy, supported by all theories, and consistent with experiment. Overall, it appears there is no major reason to be concerned.

Unfortunately, changing the target to sodium, substantial discrepancies between theory and experiment arise, and remain unresolved to date. One of the motivations for extending the CCC theory to two-centre calculations of positron-alkali scattering was to address this problem. Lugovskoy et al. [147] performed the most extensive study of this problem that included one- and two-centre calculations. Despite establishing convergence and consistency of the two approaches no improvement on previous calculations was found.

We begin by considering the total cross section for positron-sodium scattering, presented in Fig. 16. We see good agreement between various two-centre calculations, with the one-centre $\mathrm{CCC}(217,0)$ calculation behaving as expected: agreeing with $\mathrm{CCC}(116,14)$ only above

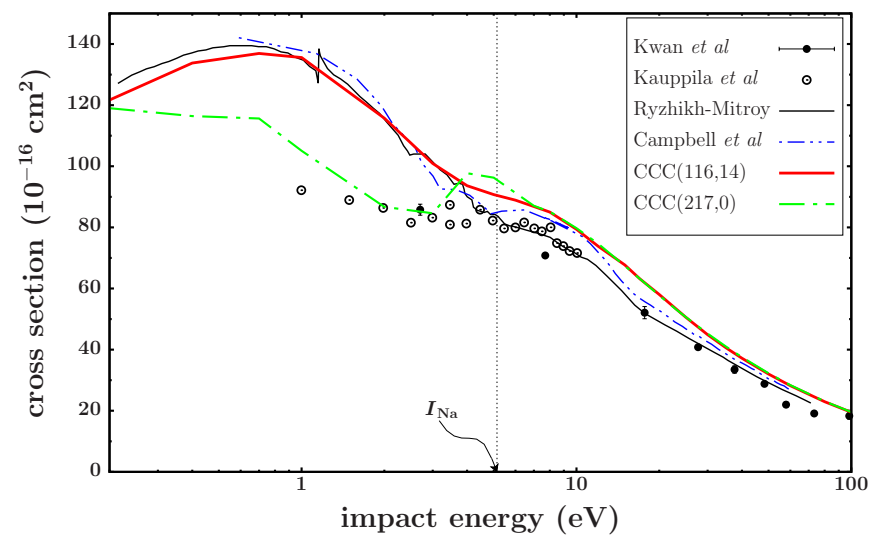

FIG. 16. Total cross section for positron-sodium scattering. The two- and one-centre CCC results of Lugovskoy et al. [147] are compared with the calculations of Ryzhikh and Mitroy [145] and Campbell et al. [111]. The experimental points are due to Kwan et al. [141] and Kauppila et al. [142].

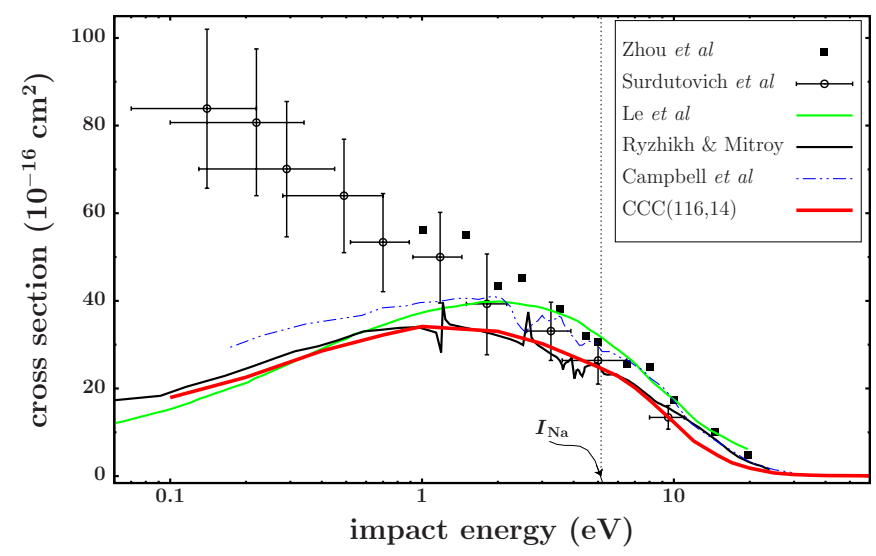

FIG. 17. Total positronium-formation cross section in $\mathrm{e}^{+}-$ $\mathrm{Na}$ scattering. Experiment is due to Surdutovich et al. 137], Zhou et al. [143], and the calculations due to Le et al. [135], Ryzhikh and Mitroy [145] and Lugovskoy et al. [147].

the ionisation threshold, and not being valid (or even convergent) below the ionisation threshold. All of the two-centre calculations are considerably above the experiment at low energies.

Curiously, the situation is reversed for the total Psformation component of the total cross section, presented in Fig. 17, where now all of the theories are considerably below the experiment. Given that the total cross section at the lowest energies considered is the sum of elastic and Ps-formation cross sections, the presented discrepancies with experiment imply that the theoretical elastic scattering cross sections are overwhelmingly high [147]. Why this would be the case remains a mystery.

Faced with the problems identified above, Lugovskoy et al. 148 considered threshold behaviour in positron scattering on alkali atoms. Following Wigner [149] we 

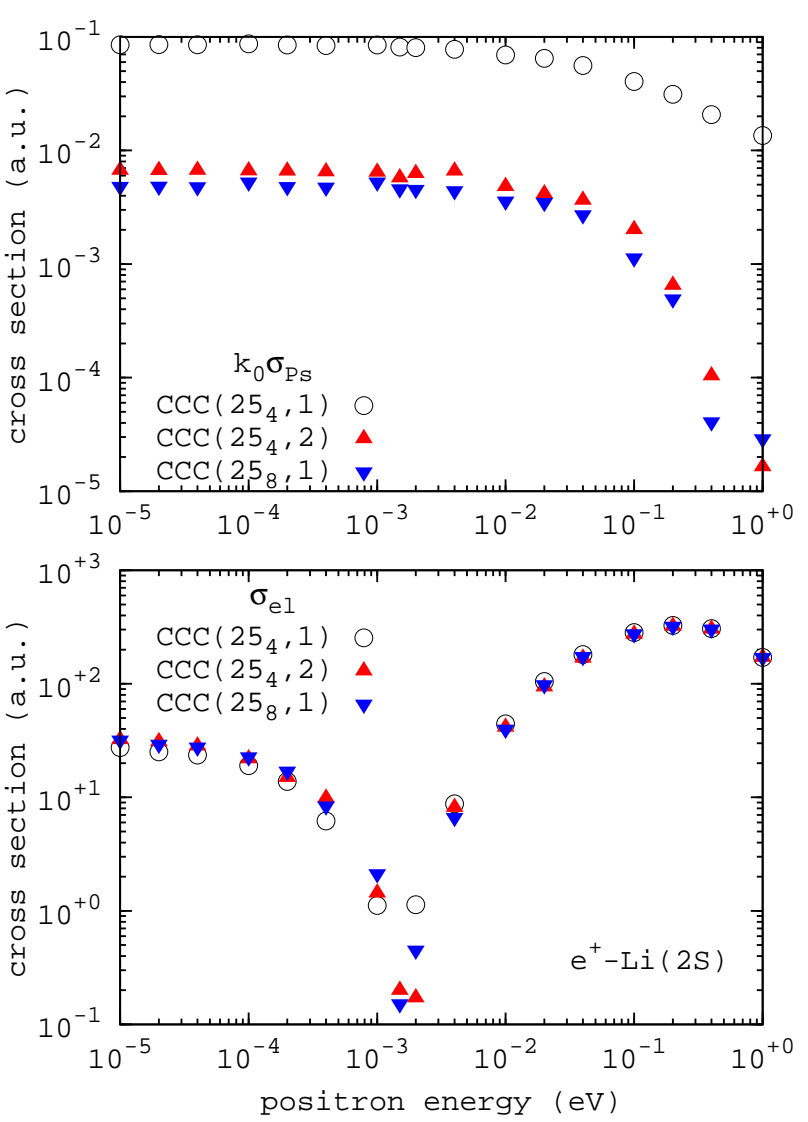

FIG. 18. Elastic $\sigma_{\mathrm{el}}$ (lower panel) and scaled Ps-formation $k_{0} \sigma_{\mathrm{Ps}}$ (upper panel) cross sections for positron-lithium scattering, as a function of energy $\left(13.6 k_{0}^{2}\right)$, for the zeroth partial wave calculated with the indicated $\operatorname{CCC}\left(N_{l_{\max }^{(\mathrm{Li})}}, N_{l_{\max }^{(\mathrm{Ps})}}\right)$ Laguerre basis parameters, as presented by Lugovskoy et al. [148].

expect exothermic reactions such as Ps formation in positron-alkali scattering to result in a cross section that tends to infinity as $1 / k_{0}$ as the positron energy $\left(k_{0}^{2}\right)$ goes to zero. This is not yet evident in Fig. 15] Fig. 17for the considered energies. Nevertheless, Lugovskoy et al. 148] did obtain the required analytical behaviour, but only in the zeroth partial wave. In Fig. 18 the Ps-formation cross section is presented for the zeroth partial wave multiplied by $k_{0}$ so as to demonstrate the expected threshold behaviour. The convergence study of Lugovskoy et al. 148] is also presented, where the effect of adding the $\operatorname{Ps}(2 s)$ state was able to be reproduced by atomic pseudostates of high orbital angular momentum. Lugovskoy et al. 148] found that higher partial waves $J$ become major contributors to $\mathrm{Ps}$ formation at energies above $10^{-3} \mathrm{eV}$, and have a threshold behaviour as $k_{0}^{2 J-1}$, and so rise rapidly with increasing energies. It is the contributions of the higher partial waves that is responsible for the behaviour in the Ps-formation cross section seen in Fig. 15.

We find the same is the case for positron-sodium scattering. In Fig. 19 we present $k_{0} \sigma_{\mathrm{Ps}}$ and $\sigma_{\mathrm{el}}$ for the zeroth partial wave, demonstrating the expected threshold be-
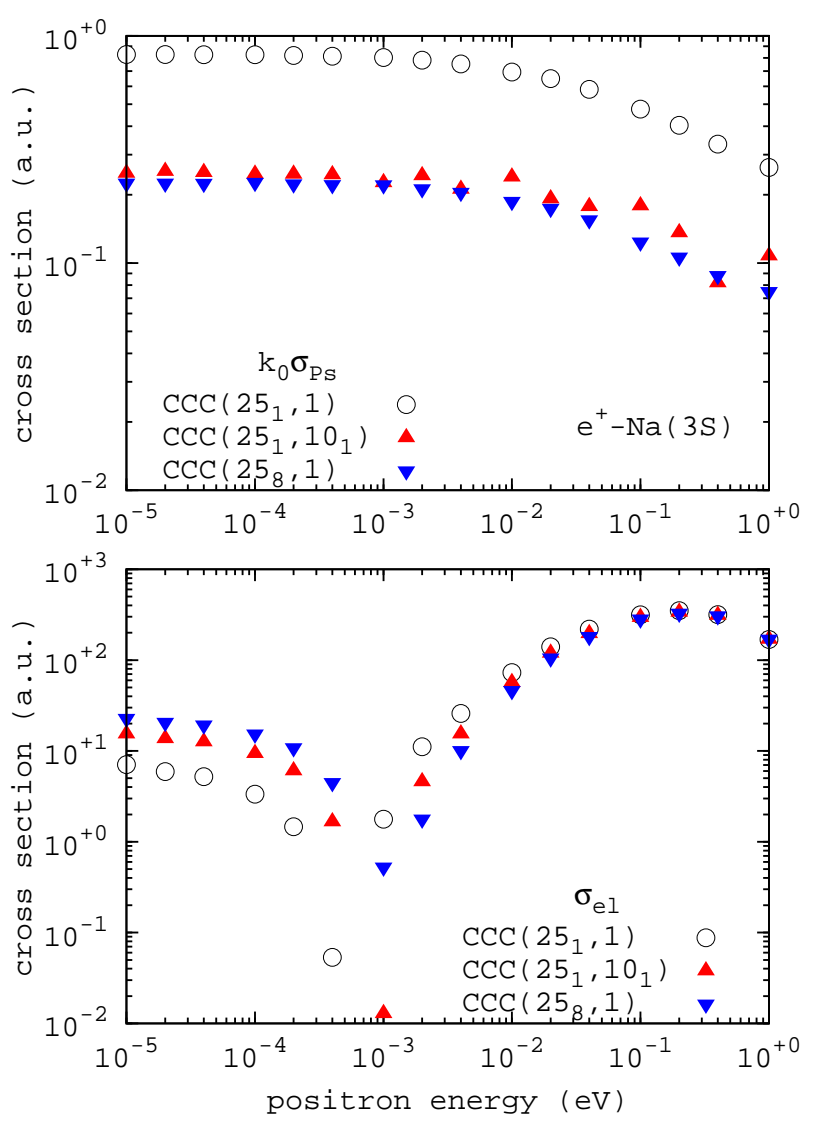

FIG. 19. The same as Fig. 18 but for positron-sodium scattering.

haviour. Details of the convergence study are discussed by Lugovskoy et al. [148]. It suffices to say that there is a range of combinations of atomic and Ps states that should yield convergent results, with such studies being considerably easier at the lower energies where there are only two open channels. As in the case of lithium the zeroth partial wave is dominant only below $10^{-3} \mathrm{eV}$, with the results presented in Fig. 17 being dominated by the higher partial waves.

Lastly, having been unable to explain the discrepancy between experiment and theory for positron-sodium scattering Lugovskoy et al. [148] also considered the positronpotassium scattering system. However, much the same behaviour as for the lighter alkalis was found, see Fig. 20 . Consequently, the discrepancy between experiment and theory for low-energy positron scattering on sodium remains unresolved.

One interesting aspect of the presented elastic cross sections for positron scattering on the alkalis are the minima. In the case of $\mathrm{Li}$ and $\mathrm{Na}$ they are at just above $0.001 \mathrm{eV}$, whereas for $\mathrm{K}$ the minimum is at around $0.04 \mathrm{eV}$. Given that in all cases we have just two channels open, elastic and Ps formation, we have no ready explanation for the minima, or their positions. The generally smaller Ps-formation cross section shows no structure in the same energy region, which is surprising given the sub- 

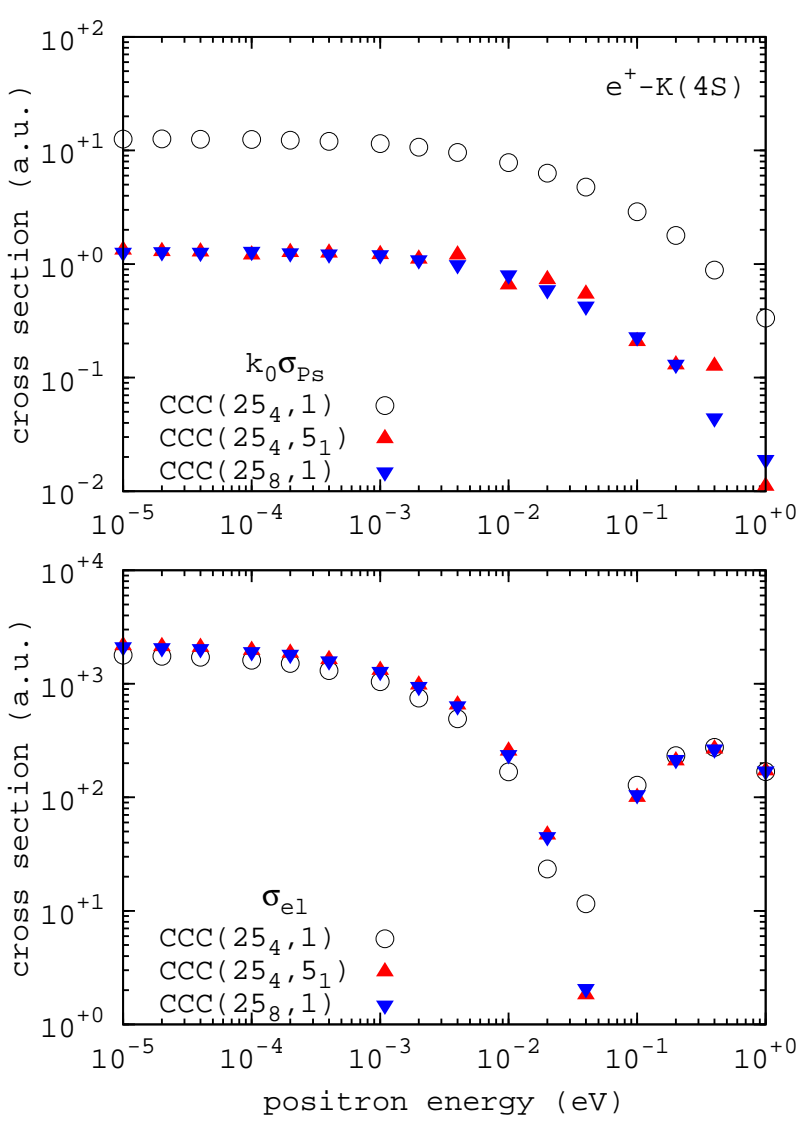

FIG. 20. The same as Fig. 18 but for positron-potassium scattering.

stantial minima in the elastic cross section.

\section{E. Magnesium and inert gases}

For experimentalists the transition from say sodium to magnesium for the purpose of positron scattering is relatively straightforward, not so in the case of theory. Two valence electrons on top of a Hartree-Fock core makes for a very complicated projectile-target combination to treat computationally. However, with an ionization energy of $7.6 \mathrm{eV}$ the single-centre approach is valid below $0.8 \mathrm{eV}$ allowing for a test of the two-centre method in this energy region. This is an important test because in the single centre approach there are no approximations associated with explicit Ps formation, and the core is fully treated by the Hartree-Fock approach rather than an equivalent local core potential approximation.

One-centre positron-magnesium CCC calculations were presented by Savage et al. [155], and two-centre ones by Utamuratov et al. [156]. The results confirmed the existence of a low-energy shape-resonance predicted earlier by Mitroy et al. [154]. The results are presented in Fig. 21. Given that the resonance is at a very low energy and that a slight energy difference in the target structure may affect its position the agreement between the theories is very encouraging. As explained in Sec. ПIA. one-centre calculations are unable to yield convergent results within the extended Ore gap (presently between $0.8 \mathrm{eV}$ and $7.6 \mathrm{eV}$ ). The unphysical structures displayed within this energy region in the calculations of Savage et al. [155] depend on the choice of basis with just one example presented.

Unfortunately, the agreement with the experiment of Stein et al. 256] for the total cross section has the unexpected feature of being good above the ionisation threshold and poor below, see Fig. 22. Given that the validity of the two-centre CCC approach should be energy independent we are unable to explain the discrepancy.

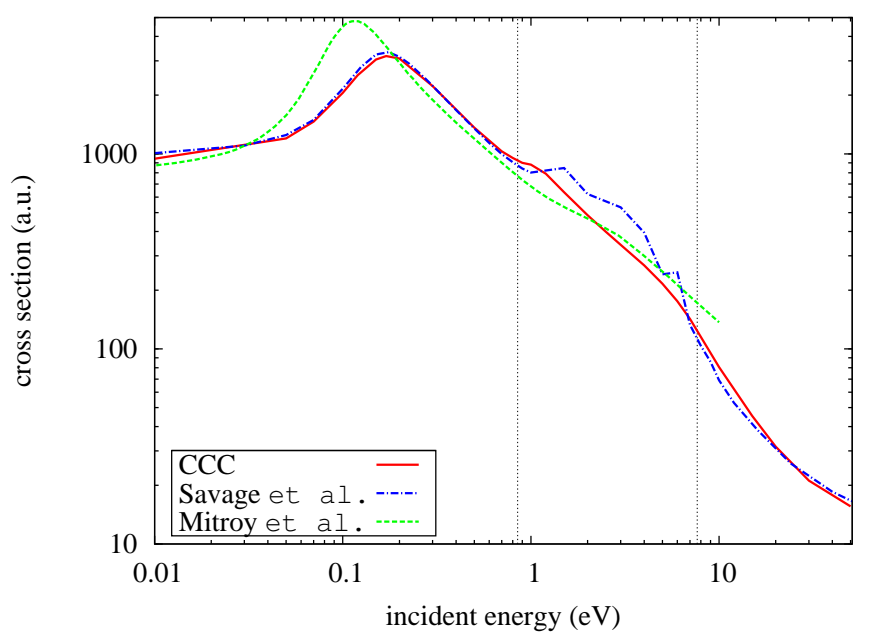

FIG. 21. $\mathrm{e}^{+}-\mathrm{Mg}$ elastic-scattering cross section. The first vertical line is at the Ps-formation threshold, the second one is at the Mg-ionization threshold. The (two-centre) CCC calculations are due to Utamuratov et al. [156], the one-centre due to Savage et al. 155], and the variational ones due to Mitroy and Bromley [257].

The Ps-formation cross section is presented in Fig. 23, where there are large experimental uncertainties. The experimental data of Surdutovich et al. 258] are preliminary estimations for the upper and the lower limits of the Ps-formation cross section. The (two-centre) CCC results are compared with the previous calculations. While there is substantial variation the theories tend to favour the upper limit estimates.

Positron scattering on inert-gas atoms has been studied using the single-centre CCC method [163]. As discussed in the introduction the complexity of adding the second (Ps) centre is immense, and has not yet been attempted within a convergent close-coupling formalism. The large ionisation thresholds mean that the single centre calculations are valid for elastic scattering on the substantial energy range below the Ps-formation threshold, as well as above the ionisation threshold, but no explicit Ps-formation cross section may be determined. This is particularly unfortunute in light of the intriguing cusplike behaviour observed by Jones et al. 21] across the Ps-formation threshold. Due to the small magnitude of 


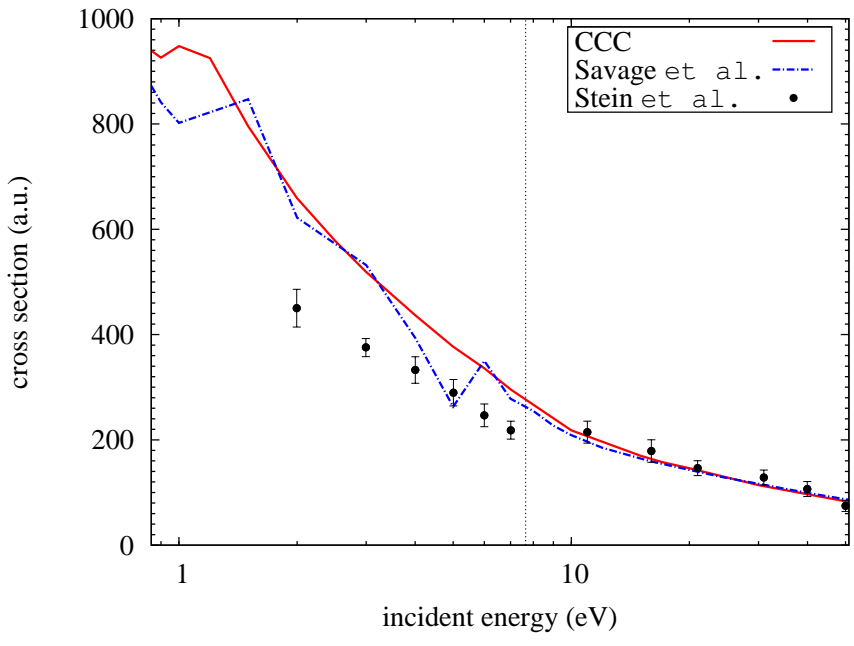

FIG. 22. $\mathrm{e}^{+}-\mathrm{Mg}$ total scattering cross section. The vertical line indicates the Mg-ionization threshold. Experimental data are due to Stein et al. 256], and the calculations are as for Fig. 21.

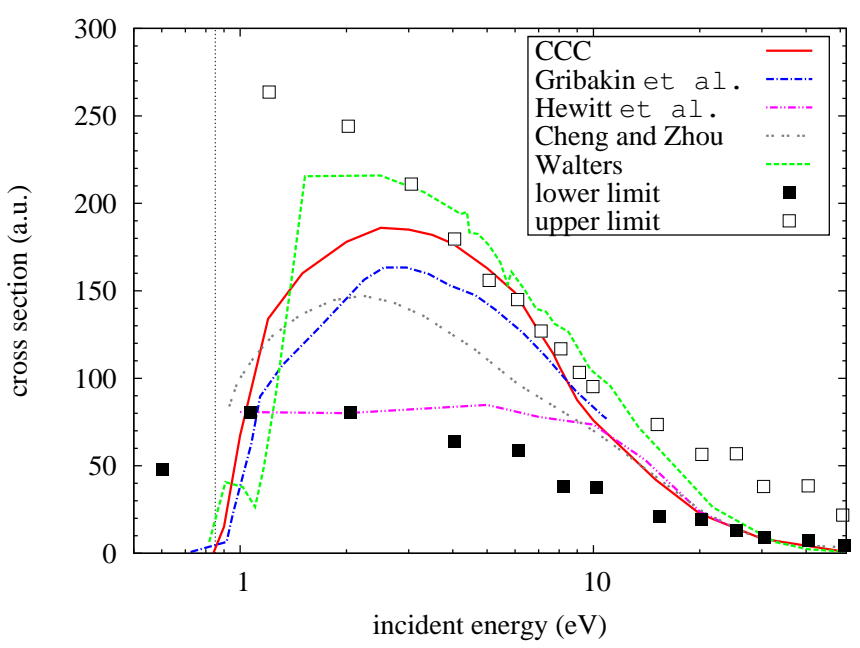

FIG. 23. $\mathrm{e}^{+}-\mathrm{Mg}$ Ps-formation cross section. Experimental data for upper and lower limits of the Ps-formation cross section are due to Surdutovich et al. 258]. The vertical line indicates the position of the Ps-formation threshold. The calculations are due to Utamuratov et al. [156], Gribakin and King [94], Hewitt et al. 159], Walters (data taken from Ref. 258]) and Cheng and Zhou 259].

the structures a highly accurate theoretical treatment is required, but does not yet exist. The experimental and theoretical situation for positron-noble gas collision systems has been recently reviewed extensively by Chiari and Zecca [11].

\section{F. Molecular hydrogen}

If we allow the internuclear separation of the two protons in $\mathrm{H}_{2}$ to be fixed then the extra complexity, relative to the helium atom, is somewhat manageable.

Various cross sections for $e^{+}-\mathrm{H}_{2}$ scattering have been recently calculated by Utamuratov et al. [199] using the two-center CCC method. This represents the most complex implementation of the two-centre formalism to date. Issues regarding convergence with Laguerre-based molecular and Ps states have been discussed in some detail. Calculations with only up to three $\operatorname{Ps}(1 s), \operatorname{Ps}(2 s)$ and $\operatorname{Ps}_{\mathrm{s}}(2 p)$ states on the Ps centre were presented above $10 \mathrm{eV}$. At lower energies the current implementation of the two-centre formalism fails to pass the internal consistency check with the single-center calculations of Zammit et al. [196]. The low energies are particularly sensitive to the approximations of the treatment of the (virtual) Ps formation in the field of the highly structured $\mathrm{H}_{2}^{+}$ion. Here we just present the key two-centre results in comparison of experiment and theory.

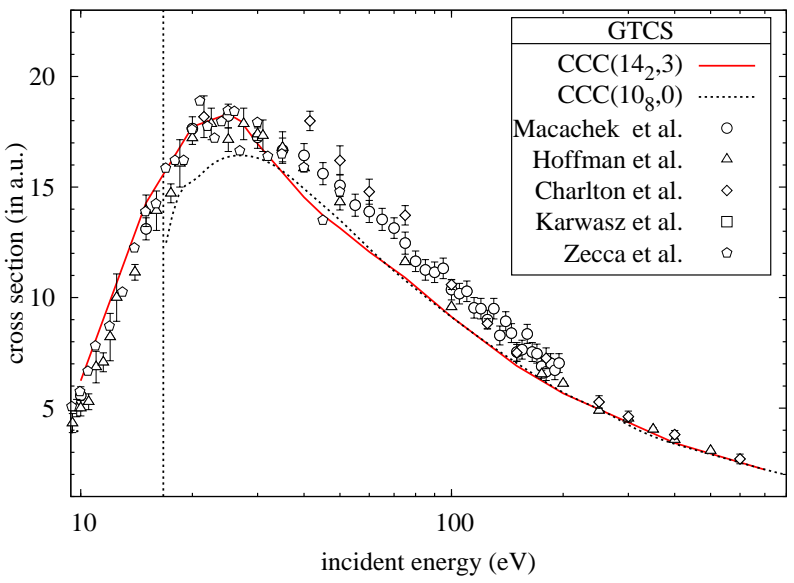

FIG. 24. The grand total cross section (GTCS) for $e^{+}-\mathrm{H}_{2}$. Experimental data are due to Machacek et al. [181], Hoffman et al. 172], Charlton et al. [173], Karwasz et al. 180] and Zecca et al. [26]. The single centre $\mathrm{CCC}\left(10_{8}, 0\right)$ results are due to Zammit et al. [196]. The two-centre CCC calculations are due to Utamuratov et al. [199].

In Fig. 24 the theoretical results are compared with the available experimental data for the grand total cross section. Good agreement between the two- and one-centre calculation above the ionisation threshold is very satisfying, even if in this energy region the theory is somewhat below experiment. Good agreement with experiment of the two-centre CCC results below the ionisation threshold, dominated by the elastic and Ps-formation cross sections, is particularly pleasing.

Fig. 25] shows the Ps-formation cross section which is a substantial component of the GTCS, particularly near its maximum around $20 \mathrm{eV}$. There is a little variation between the three CCC calculations particularly at the lower energies, with the largest CCC calculation being uniformly a little lower than experiment.

In Fig. 26 the CCC results for the direct total ionization cross section (TICS) are compared with the available 


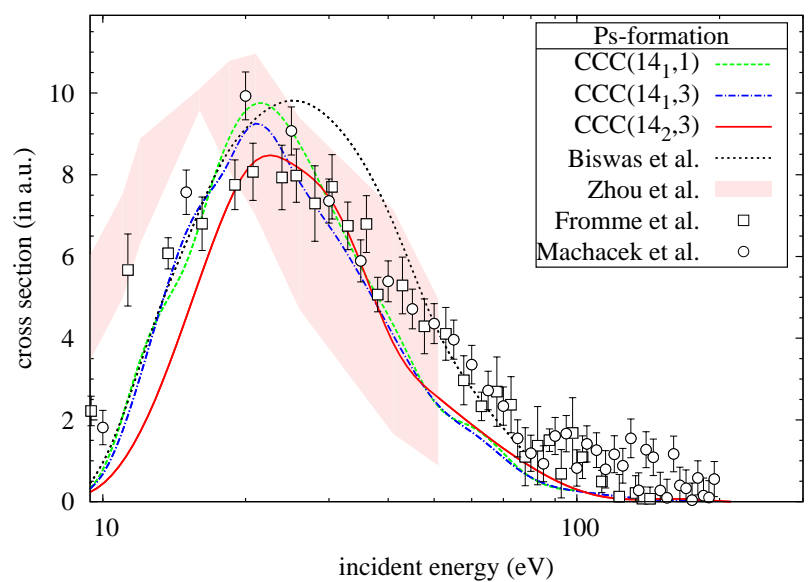

FIG. 25. The Ps-formation cross section in $e^{+}-\mathrm{H}_{2}$ collisions. Experimental data are due to Zhou et al. [178] (shaded region incorporates upper and lower limits with their uncertainties), Fromme et al. [175] and Machacek et al. [181]. Coupled static model calculations are due to Biswas et al. 186]. The CCC calculations are due to Utamuratov et al. [199].

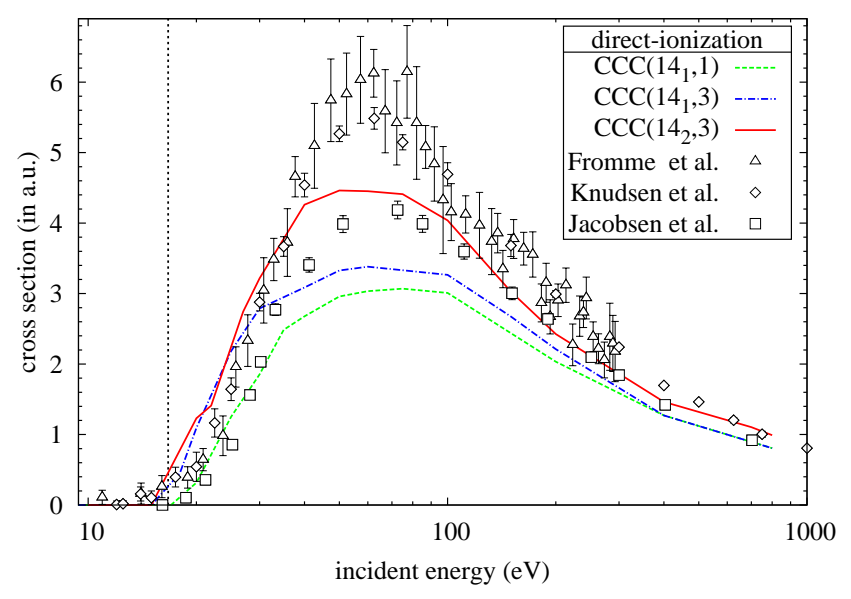

FIG. 26. The total direct-ionization cross section for $e^{+}-\mathrm{H}_{2}$ collisions. Experimental data are due to Fromme et al. [175], Knudsen et al. [85] and Jacobsen et al. 177]. The CCC calculations are due to Utamuratov et al. [199].

experimental data. The experimental data of Fromme et al. [175] and Knudsen et al. [85] are in agreement with each other but differ from measurements of Jacobsen et al. 177] between 30 and $100 \mathrm{eV}$. The largest CCC calculation $\operatorname{CCC}\left(14_{2}, 3\right)$, which contains $s-, p$ - and $d$-atomic orbitals together with the three lowest-energy Ps states, is in better agreement with the measurements of Jacobsen et al. [177]. The CCC $\left(14_{1}, 1\right)$ and $\operatorname{CCC}\left(14_{1}, 3\right)$ are systematically lower, primarily due to the absence of the $d$-atomic orbitals. Due to the unitarity of the close-coupling formalism, larger $l$-orbitals are likely to only marginally increase the TICS. Once we have con-

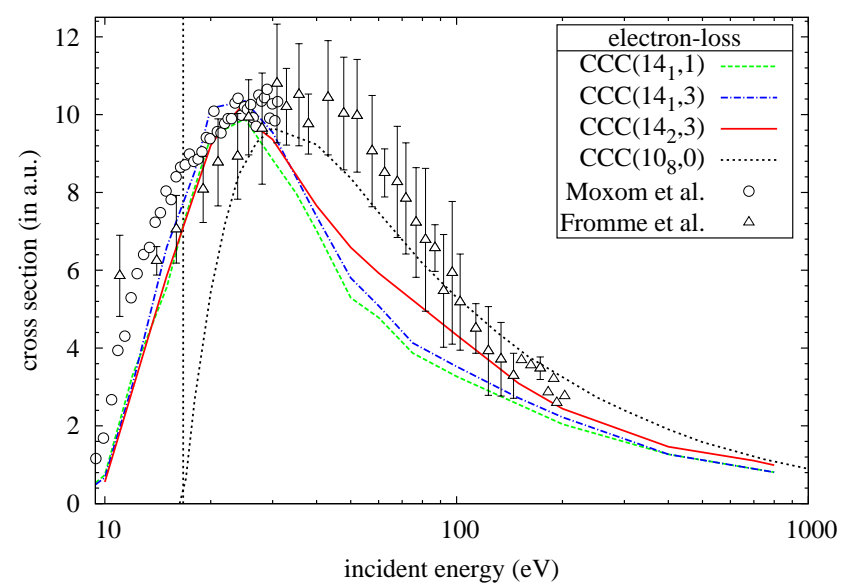

FIG. 27. The total electron-loss cross section for $e^{+}-\mathrm{H}_{2}$ collisions. Experimental data are due to Moxom et al. [176] and Fromme et al. 175]. The CCC calculations are due to Zammit et al. [196] (one-centre) and Utamuratov et al. [199] (two-centre).

vergence in the elastic scattering (large $l$-orbitals not required) the GTCS is set, with the distribution between elastic, electron-excitation, Ps-formation and total ionisation cross sections thereby being constrained [260].

Finally, the total electron-loss cross section is given in Fig. 27, and is the sum of TICS and Ps-formation cross sections. It is useful because the one-centre CCC approach is able to yield this cross section at energies above the ionisation threshold. This provides for an important internal-consistency check. We see that around $50 \mathrm{eV}$ the largest two-centre CCC calculation is somewhat lower than the one-centre calculation. Given the increase in the cross section due to the inclusion of $d$-orbitals adding $f$-orbitals would go someway to reduce the discrepancy. Increasing the Laguerre basis $N_{l}=10-l$ in the onecentre calculations would increase the cross section just above the ionisation threshold due to an improving discretisation of the continuum.

Despite the immense complexity of the positronmolecular-hydrogen scattering problem considerable progress in obtaining reasonably accurate cross sections has been made.

\section{ANTIHYDROGEN FORMATION}

As stated earlier, via Eq. (1), antihydrogen formation is effectively the time-reverse process of Ps formation upon positron-hydrogen scattering. Accordingly, it only takes place at positron energies above the $\operatorname{Ps}(n)$ formation threshold, where $n$ indicates the principal quantum number. Furthermore, we require two-centre calculations because only these have explicit Ps and H states. Given that we are interested in as large cross sections as possible, and that the exothermic transition cross sections 
go to infinity at threshold [149, 201] the primary energy range of interest is within the extended Ore gap, as given in Fig. 5.

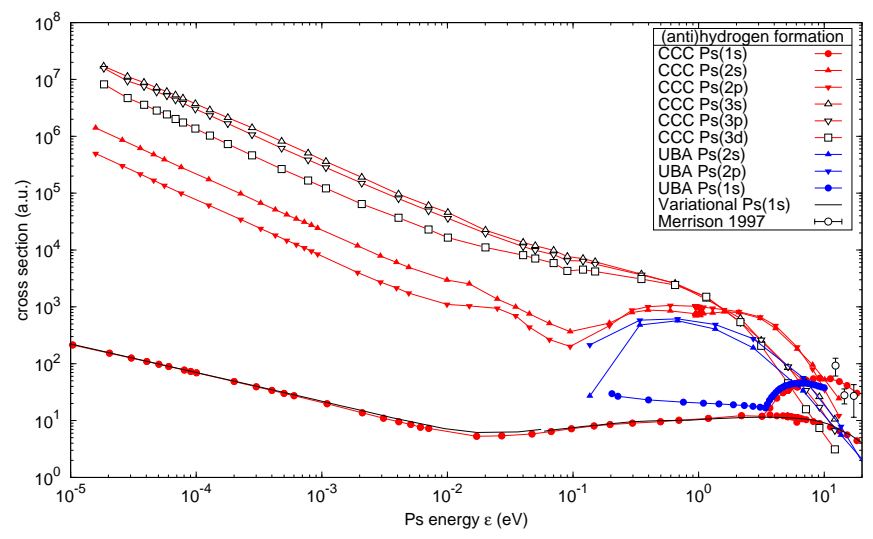

FIG. 28. Total cross sections for (anti)hydrogen formation upon positronium, in the specified initial state $n l$, scattering on (anti)protons calculated using the CCC method, first presented by Kadyrov et al. 211. For Ps(1s), the variational calculations 200, 261, 262] are for (anti)hydrogen formation in the 1s state only (CCC-calculated unconnected points presented for comparison), while the UBA calculations of Mitroy [263] and Mitroy and Stelbovics 264], and the CCC calculations generally, are for (anti)hydrogen formation in all open states. The three experimental points are due to Merrison et al. [265].

There are few calculations of antihydrogen formation that are accurate at low energies. As far as we are aware, apart from the CCC method [211, 266], only the variational approach of Humberston et al. 200 has yielded accurate results. The unitarised Born approximation (UBA) calculations 263, 267, 268 are not appropriate at low energies, and neither are first order approximations [269].

Large cross sections for antihydrogen formation occur for transitions between excited states of Ps and $\mathrm{H}$. To have these states accurate the Laguerre bases $N_{l}$ for both $\mathrm{H}$ and $\mathrm{Ps}$ need to be sufficiently large. Kadyrov et al. 211] used $N_{l}=12-l$, with the resultant energies given in Fig. 3. While it is trivial to have larger $N_{l}$ as far as the structure is concerned, the primary limiting factor is to be able to solve the resultant close-coupling equations. In Fig. 3 the positive energies correspond to the breakup of $\mathrm{H}$ and also of $\mathrm{Ps}$, and yet the two represent the same physical process. This leads to highly ill-conditioned equations. Bailey et al. [218] considered non-symmetric treatments of the two centres by dropping the Ps positive-energy states. While these also satisfy internal-consistency checks they have not proved to be more efficient in yielding convergence, with increasing $N_{l}$, in the cross sections of present interest.

\section{A. Numerical treatment of the Green's function}

A comprehensive set of antihydrogen formation cross sections for low-energy Ps incident on an antiproton has been given by Rawlins et al. [266]. They used the original numerical treatment of the Green's function in Eq. (19). In Fig. 28, we present the summary of the total antihydrogen formation cross sections for specified initial $\mathrm{Ps}(n l)$ as presented by Kadyrov et al. 211]. Excellent agreement with the variational calculations [200, 261, 262], available only for the ground states of $\mathrm{H}$ and $\mathrm{Ps}$, gives us confidence in the rest of the presented CCC results.

As predicted by Wigner [149] the ground state cross section goes to infinity as $1 / \sqrt{\varepsilon}$ at threshold, whereas for excited states this is modified to $1 / \varepsilon$ at threshold due to the long-range dipole interaction of degenerate Ps $n \geq 2$ states, as explained by Fabrikant [201]. The cross sections increase steadily with increasing principle quantum number of Ps, with the transition to the highest available principal quantum number of $\mathrm{H}$ being the most dominant contribution [266]. Consequently there is considerable motivation to push the calculations even further to larger $N_{l}$.

\section{B. Analytic treatment of the Green's function}

There is a second source of ill-conditioning of the closecoupling equations to that discussed previously. Given that we are particularly interested in low energies i.e., just above the various thresholds, the singularity in the Green's function of Eq. (19) occurs very close to zero energy. A typically used symmetric treatment on either side of the singularity results in very large positive and negative values which contribute to the ill-conditioning via precision loss.

Very recently Bray et al. 224, 225] showed that the Green's function of the coupled Lippmann-Schwinger equations (19) can be treated analytically. The method that was previously used in calculating the optical potential in the coupled-channel optical method [270, 271] was applied directly to the Green's function in the LippmannSchwinger equation (19). In doing so, they showed that they could improve on the above $N_{l}=12-l$ basis to reach $N_{l}=15-l$, and thereby consider transitions from $\operatorname{Ps}(n=4)$ states. In Fig. 29 we compare the "old" and the "new" numerical formulations and find that the latter is quite superior. In fact, for $N_{l}=15-l$ Bray et al. [225] were unable to obtain numerical stability in the original formulation with variation of integration parameters over the momentum in Eq. (19), and presented just a combination that yielded somewhat reasonable agreement with the results of the "new" numerical method. The new technique also proved to be particularly advantageous in studying threshold phenomena in positronium(anti)proton scattering [272]. 




FIG. 29. $\mathrm{H}(n s)$ formation cross sections for $n \leq 5$ in $\mathrm{p}-\mathrm{Ps}(4 s)$ scattering for the zeroth partial wave. The "old" results arise from solving the original CCC equations (19). The "new" results are due to the solution with the Green's function being treated analytically, see Bray et al. 225].

\section{CONCLUDING REMARKS}

We have presented an overview of recent developments in positron-scattering theory on several atoms and molecular hydrogen, with particular emphasis on two-centre calculations that are able to explicitly treat positronium formation. While considerable progress has been made there remain some major discrepancies between theory and experiment such as for low-energy positron-sodium scattering. Considerable technical development is still required for complicated atoms such as the heavier inert gases to incorporate Ps formation in a systematically convergent way. A general scheme for doing so for molecular targets is also required.

It is also important to state that there are still fundamental issues to be addressed in the case of breakup. While no overcompleteness has been found in two-centre calculations with near-complete bases on both centres, determining the resulting differential cross sections remains an unsolved problem. Kadyrov et al. [273] considered the simplest energy-differential cross section which describes the probablity of an electron of a certain energy being ejected. If we have contributions to this process from both centres, how do we combine them? Furthermore, diagonalising the Ps Hamiltonian yields pseudostates of positive-energy Ps rather than the energy of the individual electron or positron. It may be that the only practical way to obtain differential ionisation cross sections is to restrict the Ps centre to just negative-energy eigenstates, and obtain differential cross sections solely from the atomic centre positive-energy pseudostates, as is done for electron-impact ionisation [40]. However, this kind of approach may not be capable of describing the phenomenon of Ps formation in continuum as seen by Arcidiacono et al. 274]. This is currently under investigation.

\section{ACKNOWLEDGMENTS}

Support of the Australian Research Council and the Merit Allocation Scheme on the National Facility of the Australian Partnership for Advanced Computing is gratefully acknowledged. This work was supported by resources provided by Pawsey Supercomputing Centre with funding from the Australian Government and the Government of Western Australia. A.S.K. acknowledges partial support from the U.S. National Science Foundation under Award No. PHY-1415656.
[1] C. M. Surko, G. F. Gribakin, S. J. Buckman, Lowenergy positron interactions with atoms and molecules, J. Phys. B 38 (2005) R57.

[2] G. F. Gribakin, J. A. Young, C. M. Surko, Positronmolecule interactions: Resonant attachment, annihila- tion, and bound states, Rev. Mod. Phys. 82 (2010) 2557-2607.

[3] J. Mitroy, M. W. J. Bromley, G. G. Ryzhikh, Positron and positronium binding to atoms, J. Phys. B 35 (2002) R81. 
[4] M. Charlton, J. W. Humberston, Positron Physics, Cambridge University Press, 2001.

[5] R. J. Drachman, A general overview of positron collision physics, Nucl. Instr. and Meth. B 143 (1998) 1-10.

[6] E. Armour, The theory of low-energy positron collisions with molecules, Phys. Rep. 169 (1988) 1 - 98.

[7] M. Charlton, Experimental studies of positrons scattering in gases, Reports on Progress in Physics 48 (1985) 737.

[8] A. S. Ghosh, N. C. Sil, P. Mandal, Positron-atom and positron-molecule collisions, Physics Reports 87 (1982) 313-406.

[9] T. C. Griffith, G. R. Heyland, Experimental aspects of the study of the interaction of low-energy positrons with gases, Phys. Rep. 44 (1978) 169.

[10] G. Laricchia, S. Armitage, A. Koever, D. J. Murtagh, Ionizing Collisions by Positrons and Positronium Impact on the Inert Atoms, in: $\mathrm{E}$ Arimondo and $\mathrm{P} \mathrm{R}$ Berman and C C Lin (Ed.), Advances in atomic, molecular, and optical physics, volume 56, Elsevier Academic Press Inc, 2008, pp. 1-47.

[11] L. Chiari, A. Zecca, Recent positron-atom cross section measurements and calculations, Eur. Phys. J. D 68 (2014) 297.

[12] M. Charlton, D. P. Van Der Werf, Advances in antihydrogen physics, Science Progress 98 (2015) 34-62.

[13] W. A. Bertsche, E. Butler, M. Charlton, N. Madsen, Physics with antihydrogen, Journal of Physics B: Atomic, Molecular and Optical Physics 48 (2015) 232001.

[14] M. H. Holzscheiter, M. Charlton, M. M. Nieto, The route to ultra-low energy antihydrogen, Physics Reports 402 (2004) 1 - 101.

[15] M. Charlton, J. Eades, D. Horváth, R. Hughes, C. Zimmermann, Antihydrogen physics, Physics Reports 241 (1994) 65-117.

[16] K. Aiba, A. Igarashi, I. Shimamura, Time-delay matrix analysis of several overlapping resonances: applications to the helium atom and the positronium negative ion, Journal of Physics B: Atomic, Molecular and Optical Physics 40 (2007) F9.

[17] I. Shimamura, H. Wakimoto, A. Igarashi, Resonance states of unnatural parity in positronic atoms, Phys. Rev. A 80 (2009) 032708.

[18] A. Zecca, L. Chiari, Structures in positron atom and molecule total cross sections, Molecular Physics 113 (2015) 3615-3627.

[19] J. P. Sullivan, A. Jones, P. Caradonna, C. Makochekanwa, S. J. Buckman, A positron trap and beam apparatus for atomic and molecular scattering experiments, Rev. Sci. Instrum. 79 (2008) -.

[20] J. P. Sullivan, C. Makochekanwa, A. Jones, P. Caradonna, S. J. Buckman, High-resolution, low-energy positron scattering from helium: measurements of the total cross section, J. Phys. B 41 (2008) 081001.

[21] A. C. L. Jones, P. Caradonna, C. Makochekanwa, D. S. Slaughter, R. P. McEachran, J. R. Machacek, J. P. Sullivan, S. J. Buckman, Observation of threshold effects in positron scattering from the noble gases, Phys. Rev. Lett. 105 (2010) 073201.

[22] A. C. L. Jones, C. Makochekanwa, P. Caradonna, D. S. Slaughter, J. R. Machacek, R. P. McEachran, J. P. Sullivan, S. J. Buckman, A. D. Stauffer, I. Bray, D. V. Fursa,
Positron scattering from neon and argon, Phys. Rev. A 83 (2011) 032701.

[23] J. R. Machacek, C. Makochekanwa, A. C. L. Jones, P. Caradonna, D. S. Slaughter, R. P. McEachran, J. P. Sullivan, S. J. Buckman, S. Bellm, B. Lohmann, D. V. Fursa, I. Bray, D. W. Mueller, A. D. Stauffer, Lowenergy positron interactions with xenon, New J. Phys. 13 (2011) 125004.

[24] A. Zecca, L. Chiari, E. Trainotti, D. Fursa, I. Bray, M. Brunger, Experimental determination of the scattering length for positron scattering from krypton, Eur. Phys. J. D 64 (2011) 317-321.

[25] C. Makochekanwa, J. R. Machacek, A. C. L. Jones, P. Caradonna, D. S. Slaughter, R. P. McEachran, J. P. Sullivan, S. J. Buckman, S. Bellm, B. Lohmann, D. V. Fursa, I. Bray, D. W. Mueller, A. D. Stauffer, M. Hoshino, Low-energy positron interactions with krypton, Phys. Rev. A 83 (2011) 032721.

[26] A. Zecca, L. Chiari, A. Sarkar, K. L. Nixon, M. J. Brunger, Total cross sections for positron scattering from $\mathrm{H}_{2}$ at low energies, Phys. Rev. A 80 (2009) 032702.

[27] W. Tattersall, L. Chiari, J. R. Machacek, E. Anderson, R. D. White, M. J. Brunger, S. J. Buckman, G. Garcia, F. Blanco, J. P. Sullivan, Positron interactions with water: total elastic, total inelastic, and elastic differential cross section measurements, The Journal of Chemical Physics 140 (2014) 044320.

[28] J. R. Machacek, F. Blanco, G. Garcia, S. J. Buckman, J. P. Sullivan, Regularities in positronium formation for atoms and molecules, Journal of Physics B: Atomic, Molecular and Optical Physics 49 (2016) 064003.

[29] T. N. Rescigno, M. Baertschy, W. A. Isaacs, C. W. McCurdy, Collisional breakup in a quantum system of three charged particles, Science 286 (1999) 2474-2479.

[30] M. Baertschy, T. N. Rescigno, C. W. McCurdy, Accurate amplitudes for electron-impact ionization, Phys. Rev. A 64 (2001) 022709.

[31] P. L. Bartlett, A. T. Stelbovics, I. Bray, Propagating exterior complex scaling method for electron-hydrogen collisions, J. Phys. B 37 (2004) L69.

[32] K. Bartschat, E. T. Hudson, M. P. Scott, P. G. Burke, V. M. Burke, Electron-atom scattering at low and intermediate energies using a pseudo-state r-matrix basis, J. Phys. B 29 (1996) 115-123.

[33] K. Bartschat, The R-matrix with pseudo-states method: Theory and applications to electron scattering and photoionization, Comp. Phys. Comm. 114 (1998) 168-182.

[34] O. Zatsarinny, BSR: B-spline atomic R-matrix codes, Comp. Phys. Comm. 174 (2006) 273-356.

[35] O. Zatsarinny, K. Bartschat, Nonperturbative treatment of ionization with excitation of helium by electron impact, Phys. Rev. Lett. 107 (2011) 023203.

[36] J. Colgan, M. S. Pindzola, Double- and tripledifferential cross sections for the low-energy electronimpact ionization of hydrogen, Phys. Rev. A 74 (2006) 012713.

[37] I. Bray, A. T. Stelbovics, Explicit demonstration of the convergence of the close-coupling method for a Coulomb three-body problem, Phys. Rev. Lett. 69 (1992) 53-56.

[38] I. Bray, Close-coupling approach to coulomb three-body problems, Phys. Rev. Lett. 89 (2002) 273201.

[39] I. Bray, D. V. Fursa, A. S. Kadyrov, A. T. Stelbovics, Single ionization of helium by electron impact, Phys. Rev. A 81 (2010) 062704. 
[40] I. Bray, D. V. Fursa, A. S. Kadyrov, A. T. Stelbovics, A. S. Kheifets, A. M. Mukhamedzhanov, Electron- and photon-impact atomic ionisation, Phys. Rep. 520 (2012) $135-174$.

[41] I. B. Abdurakhmanov, A. S. Kadyrov, S. K. Avazbaev, I. Bray, Solution of the proton-hydrogen scattering problem using a quantum-mechanical two-center convergent close-coupling method, J. Phys. B 49 (2016) 115203.

[42] P. V. Reeth, J. W. Humberston, The energy dependence of the annihilation rate in positron-atom scattering, J. Phys. B 31 (1998) L231.

[43] G. Laricchia, C. Wilkin, Semiempirical approach to positron annihilation in molecules, Phys. Rev. Lett. 79 (1997) 2241-2244.

[44] C. Kurz, R. G. Greaves, C. M. Surko, Temperature dependence of positron annihilation rates in noble gases, Phys. Rev. Lett. 77 (1996) 2929-2932.

[45] H. S. W. Massey, C. B. O. Mohr, Gaseous Reactions involving Positronium, Proc. Phys. Soc. A 67 (1954) 695.

[46] W. Kohn, Variational methods in nuclear collision problems, Phys. Rev. 74 (1948) 1763-1772.

[47] C. Schwartz, Electron Scattering from Hydrogen, Phys. Rev. 124 (1961) 1468-1471.

[48] E. A. G. Armour, J. W. Humberston, Methods and programs in collisions of positrons with atoms and molecules, Phys. Rep. 204 (1991) $165-251$.

[49] A. L. Fetter, J. D. Walecka, Quantum Theory of ManyParticle Systems, McGraw-Hill, New York, 1971.

[50] G. F. Gribakin, W. A. King, The effect of virtual positronium formation on positron-atom scattering, J. Phys. B 27 (1994) 2639.

[51] G. F. Gribakin, J. Ludlow, Many-body theory of positron-atom interactions, Phys. Rev. A 70 (2004) 032720 .

[52] I. McCarthy, A. T. Stelbovics, Continuum in the atomic optical model, Phys. Rev. A 22 (1980) 502.

[53] I. E. McCarthy, A. T. Stelbovics, Momentum-space coupled-channels optical method for electron-atom scattering, Phys. Rev. A 28 (1983) 2693-2707.

[54] K. Ratnavelu, Positron Impact Ionisation of $\mathrm{H}$ and $\mathrm{He}$ Atoms: The Continuum Model, Aust. J. Phys. 44 (1991) 265.

[55] Y. Zhou, K. Ratnavelu, I. E. McCarthy, Momentumspace coupled-channel optical method for positronhydrogen scattering, Phys. Rev. A 71 (2005) 042703.

[56] J. Ma, Y. Cheng, Y. C. Wang, Y. Zhou, 2s-3s excitation for positron-metastable hydrogen collisions, Journal of Physics B: Atomic, Molecular and Optical Physics 44 (2011) 175203.

[57] P. A. Macri, J. E. Miraglia, J. Hanssen, O. A. Fojón, R. D. Rivarola, Evidence of two-channel distortion effects in positronium formation reactions, Journal of Physics B: Atomic, Molecular and Optical Physics 37 (2004) L111.

[58] L. Jiao, Y. Wang, Y. Zhou, Positronium formation for positron scattering from hydrogen: Maximum positions and scaling law, Phys. Rev. A 84 (2011) 052711.

[59] K. Jansen, S. J. Ward, J. Shertzer, J. H. Macek, Absolute cross section for positron-impact ionization of hydrogen near threshold, Phys. Rev. A 79 (2009) 022704.

[60] S. J. Ward, J. Shertzer, Hyperspherical hidden crossing method applied to ps(1s)-formation in low energy $\mathrm{e}^{+}-\mathrm{H}$,
$\mathrm{e}^{+}-\mathrm{Li}$ and $\mathrm{e}^{+}-\mathrm{Na}$ collisions, New Journal of Physics 14 (2012) 025003.

[61] N. R. Hewitt, C. J. Noble, B. H. Bransden, Positronium formation in positron hydrogen scattering, J. Phys. B 23 (1990) 4185.

[62] K. Higgins, P. G. Burke, positron scattering by atomic hydrogen including positronium formation, J. Phys. B 24 (1991) L343.

[63] J. Mitroy, Close coupling theory of positron hydrogen scattering, Aust. J. Phys. 46 (1993) 751-771.

[64] H. R. J. Walters, A. A. Kernoghan, M. T. McAlinden, C. P. Campbell, Positron Collisions with Atoms, in: P. G. Burke, C. J. Joachain (Eds.), Photon and Electron Collisions with Atoms and Molecules, Plenum Press, New York, 1997, pp. 313-345.

[65] A. A. Kernoghan, M. T. McAlinden, H. R. J. Walters, An 18-state calculation of positron-hydrogen scattering, J. Phys. B 28 (1995) 1079-1094.

[66] J. Mitroy, K. Ratnavelu, The positron-hydrogen system at low energies, J. Phys. B 28 (1995) 287-306.

[67] J. W. Humberston, Positronium formation in s-wave positron-hydrogen scattering, J. Phys. B 17 (1984) 2353.

[68] I. Bray, A. T. Stelbovics, Convergent close-coupling calculations of electron-hydrogen scattering, Phys. Rev. A 46 (1992) 6995-7011.

[69] I. Bray, A. T. Stelbovics, Convergent Close-Coupling calculations of low-energy positron-atomic-hydrogen scattering, Phys. Rev. A 48 (1993) 4787-4789.

[70] I. Bray, A. T. Stelbovics, Calculation of the total and total ionization cross sections for positron scattering on atomic hydrogen, Phys. Rev. A 49 (1994) R2224-R2226.

[71] A. A. Kernoghan, D. J. R. Robinson, M. T. McAlinden, H. R. J. Walters, Positron scattering by atomic hydrogen, J. Phys. B 29 (1996) 2089-2102.

[72] J. Mitroy, An $l^{2}$ calculation of positron-hydrogen scattering at intermediate energies, J. Phys. B 29 (1996) L263-L269.

[73] A. S. Kadyrov, I. Bray, Convergence of two-center expansions in positron-hydrogen collisions, J. Phys. B 33 (2000) L635-L640.

[74] A. S. Kadyrov, I. Bray, Two-center convergent close-coupling approach to positron-hydrogen collisions, Phys. Rev. A 66 (2002) 012710.

[75] S. Zhou, H. Li, W. E. Kauppila, C. K. Kwan, T. S. Stein, Measurements of total and positronium formation cross sections for positrons and electrons scattered by hydrogen atoms and molecules, Phys. Rev. A 55 (1997) 361-368.

[76] G. O. Jones, M. Charlton, J. Slevin, G. Laricchia, A. Kövér, M. R. Poulsen, S. N. Chormaic, Positron imact ionization of atomic hydrogen, J. Phys. B 26 (1993) L483-L488.

[77] I. F. Barna, Ionization of helium in positron collisions, Eur. Phys. J. D 30 (2004) 5.

[78] H. S. Massey, A. H. Moussa, Positronium formation in helium, Proc. Phys. Soc. (London) 77 (1961) 811.

[79] P. Mandal, S. Guha, N. C. Sil, Positron-helium collisions: Positronium formation into an arbitrary excited $s$ state, Phys. Rev. A 22 (1980) 2623-2629.

[80] L. A. Parcell, R. P. McEachran, A. D. Stauffer, Positron excitation of the $2^{1} \mathrm{~S}$ state of helium, J. Phys. B 16 (1983) 4249-4258.

[81] L. A. Parcell, R. P. McEachran, A. D. Stauffer, Positron excitation of the $2^{1} \mathrm{P}$ state of helium, J. Phys. B 20 
(1987) 2307-2315.

[82] R. Campeanu, D. Fromme, G. Kruse, R. McEachran, L. Parcell, W. Raith, G. Sinapius, A. Stauffer, Partitioning of the positron-helium total scattering cross section, J. Phys. B 20 (1987) 3557-3570.

[83] R. Campeanu, R. McEachran, A. Stauffer, Positron impact ionization of $\mathrm{He}, \mathrm{Ne}$, and Ar, Can. J. Phys. 74 (1996) 544-547.

[84] D. Fromme, G. Kruse, W. Raith, G. Sinapius, Partialcross-section measurements for ionization of helium by positron impact, Phys. Rev. Lett. 57 (1986) 3031.

[85] H. Knudsen, L. Brun-Nielsen, M. Charlton, M. R. Poulsen, Single ionization of $\mathrm{H}_{2}, \mathrm{He}, \mathrm{Ne}$ and $\mathrm{Ar}$ by positron impact, J. Phys. B 23 (1990) 3955.

[86] J. Moxom, P. Ashley, G. Laricchia, Single ionization by positron impact, Can. J. Phys. 74 (1999) 367.

[87] M. K. Srivastava, M. Kumar, A. N. Tripathi, J. Chem. Phys. 84 (1986) 4715-4717.

[88] S. Sen, P. Mandal, Positron-helium collisions: Positronium formation using distorted-wave approximation, Phys. Rev. A 80 (2009) 062714.

[89] M. Y. Amusia, N. A. Cherepkov, L. V. Chernysheva, S. G. Shapiro, Elastic scattering of slow positrons by helium, Journal of Physics B: Atomic and Molecular Physics 9 (1976) L531.

[90] B. Jaduszliwer, D. A. L. Paul, Positron-helium scattering cross sections and phase shifts below $19 \mathrm{ev}$, Can. J. Phys. 51 (1973) 1565-1572.

[91] K. F. Canter, P. G. Coleman, T. C. Griffith, G. R. Heyland, Measurement of total cross sections for low energy positron-helium collisions. (positron backscattering from metal surface), Journal of Physics B: Atomic and Molecular Physics 5 (1972) L167.

[92] E. F. Varracchio, Positron excitation of he: a random phase approximation analysis of experimental results, J. Phys. B 23 (1990) L779.

[93] V. A. Dzuba, V. V. Flambaum, G. F. Gribakin, W. A. King, Many-body calculations of positron scattering and annihilation from noble-gas atoms, J. Phys. B 29 (1996) 3151

[94] G. F. Gribakin, W. A. King, Positron scattering from mg atoms, Can. J. Phys. 74 (1996) 449-459.

[95] J. W. Humberston, The scattering of low energy positrons by helium, J. Phys. B 6 (1973) L305.

[96] P. I. Campeanu, J. W. Humberston, The scattering of p-wave positrons by helium, J. Phys. B 8 (1975) L244.

[97] R. I. Campeanu, J. W. Humberston, The scattering of s-wave positrons by helium, J. Phys. B 10 (1977) L153.

[98] P. V. Reeth, J. W. Humberston, Positronium formation in low energy s-wave positron-helium scattering, J. Phys. B 28 (1995) L511.

[99] P. V. Reeth, J. W. Humberston, Elastic scattering and positronium formation in low-energy positron-helium collisions, J. Phys. B 32 (1999) 3651.

[100] T. Mizogawa, Y. Nakayama, T. Kawaratani, M. Tosaki, , Phys. Rev. A 31 (1985) 2171-2179.

[101] T. S. Stein, W. E. Kauppila, V. Pol, J. H. Smart, G. Jesion, Measurements of total scattering cross sections for low-energy positrons and electrons colliding with helium and neon atoms, Phys. Rev. A 17 (1978) 1600-1608.

[102] Y. Cheng, Y. Zhou, Momentum-space coupled-channel calculation for positron-helium scattering, Phys. Rev. A 76 (2007) 012704.

[103] R. P. McEachran, D. L. Morgan, A. G. Ryman, A. D.
Stauffer, polarized orbital method, J. Phys. B 10 (1977) 663.

[104] A. R. Tancić, M. R. Nikolić, Optical potential approach to the slow positron scattering from helium atom, Facta Universitatis Series: Phys., Chem. and Tech. 2 (2002) $183-188$.

[105] F. A. Gianturco, R. Melissa, Optical potentials for positron scattering - a simple modeling for atomic targets, Nucl. Instr. and Meth. B 143 (1998) 81-93.

[106] D. R. Schultz, R. Olson, Single-electronremoval processes in collisions of positrons and protons with helium at intermediate velocities, Phys. Rev. A 38 (1988) 1866.

[107] R. Abrines, I. C. Percival, , Proc. Cambridge Philos. Soc. 88 (1966) 861.

[108] R. E. Olson, A. Salop, Charge-transfer and impact-ionization cross sections for fully and partially stripped positive ions colliding with atomic hydrogen, Phys. Rev. A 16 (1977) 531.

[109] K. Tökési, I. F. Barna, J. Burgdörfer, Ionization of helium in positron impact, Nucl. Instr. and Meth. B 233 (2005) 307-311.

[110] P. Caradonna, A. Jones, C. Makochekanwa, D. S. Slaughter, J. P. Sullivan, S. J. Buckman, I. Bray, D. V. Fursa, High-resolution positron scattering from helium: Grand total and positronium-formation cross sections, Phys. Rev. A 80 (2009) 032710.

[111] C. P. Campbell, M. T. McAlinden, A. A. Kernoghan, H. R. J. Walters, Positron collisions with one- and twoelectron atoms, Nucl. Instr. and Meth. B 143 (1998) 41-56.

[112] W. E. Kauppila, T. S. Stein, J. H. Smart, M. S. Dababneh, Y. K. Ho, J. P. Downing, V. Pol, Measurements of total scattering cross sections for intermediate-energy positrons and electrons colliding with helium, neon, and argon, Phys. Rev. A 24 (1981) 725.

[113] L. S. Fornari, L. M. Diana, P. G. Coleman, Positronium formation in collisions of positrons with he, ar, and $\mathrm{h}_{2}$, Phys. Rev. Lett. 51 (1983) 2276

[114] L. M. Diana, P. G. Coleman, D. L. Brooks, P. K. Pendleton, D. M. Norman, Positronium formation cross sections in he and $\mathrm{h}_{2}$ at intermediate energies, Phys. Rev. A 34 (1986) 2731-2737.

[115] N. Overton, R. J. Mills, P. G. Coleman, The energy dependence of the positronium formation cross section in helium, Journal of Physics B: Atomic, Molecular and Optical Physics 26 (1993) 3951-3958.

[116] N. R. Hewitt, C. J. Noble, B. H. Bransden, He(21S, 21P) excitation and positronium formation in positronhelium collisions at intermediate energies, J. Phys. B 25 (1992) 557-570.

[117] P. Chaudhuri, S. K. Adhikari, Differential cross sections for target excitation and positronium formation in positron-helium scattering, Phys. Rev. A 57 (1998) 984-989.

[118] P. Chaudhuri, S. K. Adhikari, Close-coupling calculations of positronium formation in positron-helium scattering, Journal of Physics B: Atomic, Molecular and Optical Physics 31 (1998) 3057.

[119] P. Chaudhuri, S. K. Adhikari, Close-coupling calculations of elastic and inelastic positron-helium scattering, Journal of Physics B: Atomic, Molecular and Optical Physics 32 (1999) 129.

[120] A. Igarashi, N. Toshima, T. Shirai, Hyperspherical coupled-channel calculations of positronium formation 
in low-energy positron-helium collisions, Phys. Rev. A 54 (1996) 5004-5009.

[121] H. Wu, I. Bray, D. V. Fursa, A. T. Stelbovics, Lowenergy positron-helium convergent close coupling calculations, J. Phys. B 37 (2004) L1-L6.

[122] H. Wu, I. Bray, D. V. Fursa, A. T. Stelbovics, Convergent close coupling calculations for positron-helium scattering at intermediate to high energies, J. Phys. B 37 (2004) 1165-1172.

[123] R. Utamuratov, A. S. Kadyrov, D. V. Fursa, I. Bray, Convergent close-coupling calculations of positronhelium collisions, Journal of Physics: Conference Series 194 (2009) 072009.

[124] R. Utamuratov, A. S. Kadyrov, D. V. Fursa, I. Bray, A two-centre convergent close-coupling approach to positron-helium collisions, J. Phys. B 43 (2010) 031001.

[125] R. Utamuratov, A. S. Kadyrov, D. V. Fursa, I. Bray, A. T. Stelbovics, Multiconfigurational two-centre convergent close-coupling approach to positron scattering on helium, J. Phys. B 43 (2010) 125203.

[126] R. Utamuratov, A. S. Kadyrov, D. V. Fursa, I. Bray, A. T. Stelbovics, Convergent close-coupling calculations of positron scattering on metastable helium, Phys. Rev. A 82 (2010) 042705.

[127] S. J. Ward, M. Horbatsch, R. P. McEachran, A. D. Stauffer, Close-coupling approach to positron scattering for lithium, sodium and potassium, Journal of Physics B: Atomic, Molecular and Optical Physics 22 (1989) 1845.

[128] K. P. Sarkar, M. Basu, A. S. Ghosh, Positron-sodium and positron-lithium scattering using cca, Journal of Physics B: Atomic, Molecular and Optical Physics 21 (1988) 1649.

[129] P. Khan, S. Dutta, A. S. Ghosh, Positron-lithium scattering using the eigenstate expansion method, Journal of Physics B: Atomic and Molecular Physics 20 (1987) 2927.

[130] M. T. McAlinden, A. A. Kernoghan, H. R. J. Walters, Positron scattering by lithium, Journal of Physics B: Atomic, Molecular and Optical Physics 30 (1997) 1543.

[131] S. Guha, A. S. Ghosh, Positron-lithium-atom collisions using the two-state approximation, Phys. Rev. A 23 (1981) 743-750.

[132] M. Basu, A. S. Ghosh, Ionisation of a lithium atom by positron impact, Journal of Physics B: Atomic and Molecular Physics 19 (1986) 1249.

[133] M. A. Abdel-Raouf, Inelastic collisions of positrons with lithium and sodium atoms, Journal of Physics B: Atomic, Molecular and Optical Physics 21 (1988) 2331.

[134] R. N. Hewitt, C. J. Noble, B. H. Bransden, The effect of positronium formation on close-coupling calculations of positron-lithium collisions, Journal of Physics B: Atomic, Molecular and Optical Physics 25 (1992) 2683.

[135] A.-T. Le, M. W. J. Bromley, C. D. Lin, Positronium formation in positron-li and positron-na collisions at low energies, Phys. Rev. A 71 (2005) 032713.

[136] A. V. Lugovskoy, A. S. Kadyrov, I. Bray, A. T. Stelbovics, Two-center convergent close-coupling calculations for positron-lithium collisions, Phys. Rev. A 82 (2010) 062708.

[137] E. Surdutovich, J. M. Johnson, W. E. Kauppila, C. K. Kwan, T. S. Stein, Positronium formation in $e^{+}-\mathrm{Li}$ and $e^{+}-\mathrm{Na}$ collisions at low energies, Phys. Rev. A 65
(2002) 032713.

[138] S. Guha, P. Mandal, Model potential approach for positron-atom collisions. i. positronium formation in ground state in alkali atoms na, $\mathrm{k}, \mathrm{rb}$ and cs using the distorted-wave approximation, Journal of Physics B: Atomic and Molecular Physics 13 (1980) 1919.

[139] S. N. Nahar, J. M. Wadehra, Positronium formation from li and na atoms by use of pseudopotentials, Phys. Rev. A 35 (1987) 4533-4540.

[140] R. N. Hewitt, C. J. Noble, B. H. Bransden, Positron collisions with alkali atoms at low and intermediate energies, Journal of Physics B: Atomic, Molecular and Optical Physics 26 (1993) 3661.

[141] C. K. Kwan, W. E. Kauppila, R. A. Lukaszew, S. P. Parikh, T. S. Stein, Y. J. Wan, M. S. Dababneh, Total cross section measurements for positrons and electrons scattered by sodium and potassium atoms, Phys. Rev. A 44 (1991) 1620-1635.

[142] W. E. Kauppila, C. K. Kwan, T. S. Stein, S. Zhou, Evidence for channel-coupling effects in positron scattering by sodium and potassium atoms, Journal of Physics B: Atomic, Molecular and Optical Physics 27 (1994) L551.

[143] S. Zhou, S. P. Parikh, W. E. Kauppila, C. K. Kwan, D. Lin, A. Surdutovich, T. S. Stein, Measurements of positronium formation cross sections for positron scattering by k, na, and ar atoms, Phys. Rev. Lett. 73 (1994) 236-239.

[144] J. Mitroy, K. Ratnavelu, Close coupling theory of positron scattering from alkali atoms, Australian Journal of Physics 47 (1994) 721-742.

[145] G. Ryzhikh, J. Mitroy, Positron scattering from atomic sodium, Journal of Physics B: Atomic, Molecular and Optical Physics 30 (1997) 5545.

[146] Y. Ke, Y. Zhou, G. Nan, Optical model for positronium formation in $e^{+}-n a$ collision, Phys. Rev. A 70 (2004) 024702 .

[147] A. V. Lugovskoy, A. S. Kadyrov, I. Bray, A. T. Stelbovics, Two-center convergent-close-coupling calculations for positron-sodium collisions, Phys. Rev. A 85 (2012) 034701.

[148] A. V. Lugovskoy, R. Utamuratov, A. S. Kadyrov, A. T. Stelbovics, I. Bray, Threshold behavior of positronium formation in positron alkali-metal scattering, Phys. Rev. A 87 (2013) 042708.

[149] E. P. Wigner, On the behavior of cross sections near thresholds, Phys. Rev. 73 (1948) 1002-1009.

[150] M. T. McAlinden, A. A. Kernoghan, H. R. J. Walters, Positron scattering by potassium, Journal of Physics B: Atomic, Molecular and Optical Physics 29 (1996) 555.

[151] J. Chin, K. Ratnavelu, Y. Zhou, Positron-rubidium atom scattering at intermediate and high energies, The European Physical Journal D 66 (2012) 82.

[152] M. Umair, S. Jonsell, Resonances with natural and unnatural parities in positron-sodium scattering, Phys. Rev. A 92 (2015) 012706.

[153] M. Umair, S. Jonsell, Resonances in positron-potassium $(\mathrm{e}+-\mathrm{k})$ system with natural and unnatural parities, Journal of Physics B: Atomic, Molecular and Optical Physics 49 (2016) 015004.

[154] J. Mitroy, J. Y. Zhang, M. W. J. Bromley, S. I. Young, Positron-atom scattering using pseudostate energy shifts, Phys. Rev. A 78 (2008) 012715.

[155] J. S. Savage, D. V. Fursa, I. Bray, Convergent closecoupling calculations of positron-magnesium scattering, 
Phys. Rev. A 83 (2011) 062709.

[156] R. Utamuratov, D. V. Fursa, A. S. Kadyrov, A. V. Lugovskoy, J. S. Savage, I. Bray, Two-center convergentclose-coupling calculations of positron scattering on magnesium, Phys. Rev. A 86 (2012) 062702.

[157] R. I. Campeanu, R. P. McEachran, L. A. Parcell, A. D. Stauffer, Positron scattering from magnesium, Nuclear Instruments and Methods in Physics Research Section B 143 (1998) 21-26.

[158] M. W. J. Bromley, J. Mitroy, G. Ryzhikh, The elastic scattering of positrons from beryllium and magnesium in the low-energy region, J. Phys. B: At. Mol. Opt. Phys. 31 (1998) 4449-4458.

[159] R. N. Hewitt, C. Noble, B. H. Bransden, C. J. Joachain, Coupled-channel calculations of $\mathrm{e}^{+}-\mathrm{Mg}$ scattering, Can. J. Phys. 74 (1996) 559-563.

[160] A. Zecca, L. Chiari, Structures in positronatom and molecule total cross sections, Molecular Physics 113 (2015) 3615-3627.

[161] O. Zatsarinny, K. Bartschat, B-spline breit-pauli rmatrix calculations for electron collisions with neon atoms, J. Phys. B 37 (2004) 2173-2189.

[162] O. Zatsarinny, K. Bartschat, Benchmark calculations for near-threshold electron-impact excitation of krypton and xenon atoms, J. Phys. B 43 (2010) 074031.

[163] D. V. Fursa, I. Bray, Convergent close-coupling method for positron scattering from noble gases, New J. Phys. 14 (2012) 035002.

[164] S. Chen, R. P. McEachran, A. D. Stauffer, Ab initio optical potentials for elastic electron and positron scattering from the heavy noble gases, J. Phys. B 41 (2008) 025201.

[165] L. A. Parcell, R. P. McEachran, A. D. Stauffer, Positron scattering from xenon, Nucl. Instr. and Meth. B 192 (2002) 180-184.

[166] M. T. McAlinden, H. R. J. Walters, , Hyperfine Interact. 73 (1992) 65.

[167] S. Gilmore, J. E. Blackwood, H. R. J. Walters, Poistronium formation in positron-noble gas collisions, Nucl. Instr. and Meth. B 221 (2004) 129-133.

[168] D. G. Green, J. A. Ludlow, G. F. Gribakin, Positron scattering and annihilation on noble-gas atoms, Phys. Rev. A 90 (2014) 032712.

[169] I. I. Fabrikant, G. F. Gribakin, Similarity between positronium-atom and electron-atom scattering, Phys. Rev. Lett. 112 (2014) 243201.

[170] S. J. Brawley, S. Armitage, J. Beale, D. E. Leslie, A. I. Williams, G. Laricchia, Electron-like scattering of positronium, Science 330 (2010) 789-789.

[171] L. A. Poveda, A. Dutra, J. R. Mohallem, D. Assafrão, Model-potential approach to positron elastic scattering from noble gases, Phys. Rev. A 87 (2013) 052702.

[172] K. R. Hoffman, M. S. Dababneh, Y. F. Hsieh, W. E. Kauppila, V. Pol, J. H. Smart, T. S. Stein, Total-crosssection measurements for positrons and electrons colliding with $\mathrm{h}_{2}, \mathrm{n}_{2}$, and $\mathrm{co}_{2}$, Phys. Rev. A 25 (1982) 1393-1403.

[173] M. Charlton, T. C. Griffith, G. R. Heyland, G. L. Wright, Total scattering cross sections for low-energy positrons in the molecular gases $\mathrm{h}_{2}, \mathrm{n}_{2}, \mathrm{co}_{2}, \mathrm{O}_{2}$ and $\mathrm{ch}_{4}$, J. Phys. B 16 (1983) 323.

[174] T. G. Griffith, Positronium formation cross-sections in various gases, in: J. W. Humberston, M. R. C. McDowell (Eds.), Positron scattering in gases, Plenum Press,
New York, 1984, pp. 53-63.

[175] D. Fromme, G. Kruse, W. Raith, G. Sinapius, Ionisation of molecular hydrogen by positrons, J. Phys. B 21 (1988) L261.

[176] J. Moxom, G. Laricchia, M. Charlton, Total ionization cross sections of $\mathrm{He}, \mathrm{H}_{2}$ and Ar by positron impact, J. Phys. B 26 (1993) L367.

[177] F. M. Jacobsen, N. P. Frandsen, H. Knudsen, U. Mikkelsen, Non-dissociative single ionization of molecular hydrogen by electron and positron impact, J. Phys. B 28 (1995) 4675.

[178] S. Zhou, H. Li, W. E. Kauppila, C. K. Kwan, T. S. Stein, Measurements of total and positronium formation cross sections for positrons and electrons scattered by hydrogen atoms and molecules, Phys. Rev. A 55 (1997) 361-368.

[179] J. P. Sullivan, S. J. Gilbert, J. P. Marler, L. D. Barnes, S. J. Buckman, C. M. Surko, Low energy positron scattering and annihilation studies using a high resolution trap-based beam, Nucl. Instr. and Meth. B 192 (2002) $3-16$.

[180] G. P. Karwasz, D. Pliszka, R. S. Brusa, Total cross sections for positron scattering in argon, nitrogen and hydrogen below $20 \mathrm{ev,} \mathrm{Nucl.} \mathrm{Instr.} \mathrm{and} \mathrm{Meth.} \mathrm{B} 247$ (2006) 68.

[181] J. R. Machacek, E. K. Anderson, C. Makochekanwa, S. J. Buckman, J. P. Sullivan, Positron scattering from molecular hydrogen, Phys. Rev. A 88 (2013) 042715.

[182] D. P. Sural, S. C. Mukherjee, Positronhydrogen molecule scattering considering the positroniumformation channel, Physica 49 (1991) 249-260.

[183] R. W. Bussard, R. Ramaty, R. J. Drachman, The annihilation of galactic positrons, Astrophysical Journal 228 (1979) 928.

[184] A. Ray, P. P. Ray, B. C. Saha, Positronium formation in positron-hydrogen-molecule collisions, Journal of Physics B: Atomic and Molecular Physics 13 (1980) 4509.

[185] G. Danby, J. Tennyson, Differential cross sections for elastic positron-h 2 collisions using the r-matrix method, J. Phys. B 23 (1990) 1005.

[186] P. K. Biswas, T. Mukherjee, A. S. Ghosh, Groundstate ps formation in e $+-\mathrm{h} 2$ scattering using the fba, J. Phys. B 24 (1991) 2601.

[187] P. K. Biswas, M. Basu, A. S. Ghosh, J. W. Darewych, Positronium formation in the $\mathrm{n}=2$ states in $\mathrm{e}+-\mathrm{h} 2$ scattering, J. Phys. B 24 (1991) 3507.

[188] D. D. Reid, W. B. Klann, J. M. Wadehra, Scattering of low- to intermediate-energy positrons from molecular hydrogen, Phys. Rev. A 70 (2004) 062714.

[189] F. Arretche, R. da Costa, S. d'A. Sanchez, A. Hisi, E. de Oliveira, M. do N. Varella, M. Lima, Similarities and differences in e+- - molecule scattering: Applications of the schwinger multichannel method, Nuclear Instruments and Methods in Physics Research Section B: Beam Interactions with Materials and Atoms 247 (2006) 13 - 19. Proceedings of the XIII International Workshop on Low-Energy Positron and Positronium Physics.

[190] F. Arretche, M. A. P. Lima, Electronic excitation of $h_{2}$ by positron impact, Phys. Rev. A 74 (2006) 042713.

[191] S. d. A. Sanchez, M. A. P. Lima, The influence of f-type function in positron-he/positron-h2 scattering with the schwinger multichannel method, Nucl. Instr. and Meth. B 266 (2008) 447-451. 
[192] T. Mukherjee, N. K. Sarkar, Ro-vibrational close coupling study of positron-hydrogen molecule scattering using the parameter-free model correlation polarization potential, J. Phys. B 41 (2008) 125201.

[193] R. Zhang, K. L. Baluja, J. Franz, J. Tennyson, Positron collisions with molecular hydrogen: cross sections and annihilation parameters calculated using the r-matrix with pseudo-states method, J. Phys. B 44 (2011) 035203.

[194] W. Tenfen, K. T. Mazon, S. E. Michelin, F. Arretche, Low-energy elastic positron cross sections for $h_{2}$ and $n_{2}$ using an ab initio target polarization, Phys. Rev. A 86 (2012) 042706.

[195] K. Fedus, J. Franz, G. P. Karwasz, Positron scattering on molecular hydrogen: Analysis of experimental and theoretical uncertainties, Phys. Rev. A 91 (2015) 062701.

[196] M. C. Zammit, D. V. Fursa, I. Bray, Convergentclose-coupling formalism for positron scattering from molecules, Phys. Rev. A 87 (2013) 020701.

[197] I. B. Abdurakhmanov, A. S. Kadyrov, D. V. Fursa, I. Bray, Target structure-induced suppression of the ionization cross section for low-energy antiprotonmolecular hydrogen collisions: Theoretical confirmation, Phys. Rev. Lett. 111 (2013) 173201.

[198] I. B. Abdurakhmanov, A. S. Kadyrov, D. V. Fursa, S. K. Avazbaev, I. Bray, Close-coupling approach to antiproton-impact breakup of molecular hydrogen, Phys. Rev. A 89 (2014) 042706.

[199] R. Utamuratov, A. S. Kadyrov, D. V. Fursa, M. C. Zammit, I. Bray, Two-center close-coupling calculations of positron-molecular-hydrogen scattering, Phys. Rev. A 92 (2015) 032707.

[200] J. W. Humberston, M. Charlton, F. M. Jacobson, B. I. Deutch, On antihydrogen formation in collisions of antiprotons with positronium, Journal of Physics B: Atomic and Molecular Physics 20 (1987) L25.

[201] I. I. Fabrikant, The violation of the wigner law in a problem with degenerate channels coupled by long-range potential, J. Phys. B 7 (1974) L259.

[202] M. Doser, et al., Exploring the WEP with a pulsed cold beam of antihydrogen, Class. Quant. Grav. 29 (2012) 184009 .

[203] D. Krasnický, et al., Measuring $\bar{g}$ with aeḡis, progress and perspectives, Int J Mod Phys Conf Ser 30 (2014) 1460262.

[204] G. Testera, S. Aghion, C. Amsler, A. Ariga, T. Ariga, A. Belov, G. Bonomi, P. Braunig, J. Bremer, R. Brusa, L. Cabaret, M. Caccia, R. Caravita, F. Castelli, G. Cerchiari, K. Chlouba, S. Cialdi, D. Comparat, G. Consolati, S. Curreli, A. Demetrio, H. Derking, L. D. Noto, M. Doser, A. Dudarev, A. Ereditato, R. Ferragut, A. Fontana, S. Gerber, M. Giammarchi, A. Gligorova, S. Gninenko, S. Haider, S. Hogan, H. Holmestad, T. Huse, E. J. Jordan, J. Kawada, A. Kellerbauer, M. Kimura, D. Krasnický, V. Lagomarsino, S. Lehner, C. Malbrunot, S. Mariazzi, V. Matveev, Z. Mazzotta, G. Nebbia, P. Nedelec, M. Oberthaler, N. Pacifico, L. Penasa, V. Petracek, C. Pistillo, F. Prelz, M. Prevedelli, L. Ravelli, C. Riccardi, O. M. Røhne, S. Rosenberger, A. Rotondi, H. Sandaker, R. Santoro, P. Scampoli, L. Semeria, M. Simon, M. Spacek, J. Storey, I. M. Strojek, M. Subieta, E. Widmann, P. Yzombard, S. Zavatarelli, J. Zmeskal, The aegis ex- periment, Hyperfine Interactions 233 (2015) 13-20.

[205] G. Chardin, et al., Proposal to measure the Gravitational Behaviour of Antihydrogen at Rest, Technical Report CERN-SPSC-2011-029. SPSC-P-342, CERN, Geneva, 2011.

[206] D. P. van der Werf, The gbar experiment, Int J Mod Phys Conf Ser 30 (2014) 1460263.

[207] P. Pérez, et al., The gbar antimatter gravity experiment, Hyperfine Interactions 233 (2015) 21-27.

[208] C. H. Storry, A. Speck, D. L. Sage, N. Guise, G. Gabrielse, D. Grzonka, W. Oelert, G. Schepers, T. Sefzick, H. Pittner, M. Herrmann, J. Walz, T. W. Hänsch, D. Comeau, E. A. Hessels, First laser-controlled antihydrogen production, Phys. Rev. Lett. 93 (2004) 263401.

[209] E. Widmann, et al., Measurement of the hyperfine structure of antihydrogen in a beam, Hyperfine Interact. 215 (2013) 1.

[210] C. Amole, et al., Observation of hyperfine transitions in trapped ground-state antihydrogen, Hyperfine Interactions 233 (2015) 41-46.

[211] A. S. Kadyrov, C. M. Rawlins, A. T. Stelbovics, I. Bray, M. Charlton, Antihydrogen formation via antiproton scattering with excited positronium, Phys. Rev. Lett. 114 (2015) 183201.

[212] A. M. Mukhamedzhanov, A. S. Kadyrov, F. Pirlepesov, Leading asymptotic terms of the three-body coulomb scattering wave function, Phys. Rev. A 73 (2006) 012713.

[213] A. S. Kadyrov, A. M. Mukhamedzhanov, A. T. Stelbovics, I. Bray, F. Pirlepesov, Asymptotic behaviour of the Coulomb three-body scattered wave, Phys. Rev. A 68 (2003) 022703.

[214] A. S. Kadyrov, I. Bray, Convergent close-coupling calculations of positron-hydrogen S-wave model, Nucl. Instr. and Meth. B 171 (2000) 119-125.

[215] A. S. Kadyrov, I. Bray, Expansion approach to a three-body problem: model positron-hydrogen scattering, Nuclear Physics A 684 (2001) 669-671.

[216] S. G. Mikhlin, Variational Methods in Mathematical Physics, Pergamon, Oxford, 1964.

[217] A. S. Kadyrov, I. Bray, A. T. Stelbovics, Nearthreshold positron-impact ionization of atomic hydrogen, Phys. Rev. Lett. 98 (2007) 263202.

[218] J. J. Bailey, A. S. Kadyrov, I. Bray, Internal consistency in positron-hydrogen-scattering calculations, Phys. Rev. A 91 (2015) 012712.

[219] A. S. Kadyrov, A. V. Lugovskoy, R. Utamuratov, I. Bray, Benchmark calculation of hydrogen (antihydrogen) formation at rest in positronium-proton (antiproton) scattering, Phys. Rev. A 87 (2013) 060701.

[220] A. S. Kadyrov, I. Bray, A. T. Stelbovics, B. Saha, Direct solution of the three-dimensional Lippmann-Schwinger equation, J. Phys. B 38 (2005) 509-515.

[221] A. S. Kadyrov, I. Bray, A. T. Stelbovics, On-shell coupled-channel approach to proton-hydrogen collisions without partial-wave expansion, Phys. Rev. A 73 (2006) 012710.

[222] A. S. Kadyrov, I. B. Abdurakhmanov, I. Bray, A. T. Stelbovics, Three-dimensional integral-equation approach to proton- and antiproton-hydrogen collisions, Phys. Rev. A 80 (2009) 022704.

[223] D. A. Varshalovich, A. N. Moskalev, V. K. Khersonskii, Quantum theory of angular momentum, World Scien- 
tific Pub., Philadelphia, 1st edition, 1988.

[224] A. W. Bray, I. B. Abdurakhmanov, A. S. Kadyrov, D. V. Fursa, I. Bray, Solving close-coupling equations in momentum space without singularities, Comp. Phys. Comm. 196 (2015) 276 - 279.

[225] A. W. Bray, I. B. Abdurakhmanov, A. S. Kadyrov, D. V. Fursa, I. Bray, Solving close-coupling equations in momentum space without singularities ii, Comp. Phys. Comm. 203 (2016) $147-151$.

[226] D. V. Fursa, I. Bray, Calculation of electron-helium scattering, Phys. Rev. A 52 (1995) 1279-1298.

[227] I. Bray, Convergent close-coupling method for the calculation of electron scattering on hydrogen-like targets, Phys. Rev. A 49 (1994) 1066-1082.

[228] J. B. Furness, I. E. McCarthy, Semiphenomenological optical model for electron scattering on atoms, Journal of Physics B: Atomic and Molecular Physics 6 (1973) 2280 .

[229] B. J. Albright, K. Bartschat, P. R. Flicek, Core potentials for quasi-one-electron systems, Journal of Physics B: Atomic, Molecular and Optical Physics 26 (1993) 337.

[230] J. Migdalek, W. E. Baylis, Nonadjustable local model potentials for the exchange interaction between valence and core electrons, Phys. Rev. A 24 (1981) 649-655.

[231] D. V. Fursa, I. Bray, Convergent close-coupling calculations of electron scattering on helium-like atoms and ions; electron-beryllium scattering, J. Phys. B 30 (1997) 5895-5913.

[232] D. V. Fursa, I. Bray, Excitation of the $3^{1} p$ state of magnesium by electron impact from the ground state, Phys. Rev. A 63 (2001) 032708.

[233] W. A. Isaacs, M. A. Morrison, Analytic born completion in the calculation of electron-molecule differential cross sections, Phys. Rev. A 53 (1996) 4215-4221.

[234] H. Yalim, D. Cvejanovic, A. Crowe, 1s-2p excitation of atomic hydrogen by electron impact studied using the angular correlation technique, Phys. Rev. Lett. 79 (1997) 2951-2954.

[235] R. W. O’Neill, P. J. M. van der Burgt, D. Dziczek, P. Bowe, S. Chwirot, J. A. Slevin, Polarization correlation measurements of electron impact excitation of $\mathrm{H}(2 \mathrm{p})$ at $54.4 \mathrm{eV}$, Phys. Rev. Lett. 80 (1998) 1630-1633.

[236] J. F. Williams, A. G. Mikosza, Electron\&ndash;photon angular correlations from the electron impact excitation of the $2 \mathrm{~s}$ and $2 \mathrm{p}$ electronic configurations of atomic hydrogen, J. Phys. B 39 (2006) 4339-4340.

[237] A. S. Kheifets, I. Bray, Calculation of circular dichroism in helium double photoionization, Phys. Rev. Lett. 81 (1998) 4588-4591.

[238] M. Achler, V. Mergel, L. Spielberger, R. Dorner, Y. Azuma, H. Schmidt-Böcking, Photo double ionization of He by circular and linear polarized single-photon absorption, J. Phys. B 34 (2001) 965-981.

[239] A. S. Kheifets, I. Bray, Convergent close coupling calculations of helium double photoionization triply differential cross sections, J. Phys. B 31 (1998) L447-L453.

[240] I. Bray, D. V. Fursa, A. S. Kheifets, A. T. Stelbovics, Topical review: Electrons and photons colliding with atoms: Development and application of the convergent close-coupling method, J. Phys. B 35 (2002) R117R146.

[241] I. Bray, J. J. Bailey, D. V. Fursa, A. S. Kadyrov, R. Utamuratov, Internal consistency in the close-coupling ap- proach to positron collisions with atoms, The European Physical Journal D 70 (2016) 1-6.

[242] W. Ihra, J. H. Macek, F. Mota-Furtado, P. F. O'Mahony, Threshold law for positron impact ionization of atoms, Phys. Rev. Lett. 78 (1997) 4027-4030.

[243] J. M. Rost, E. J. Heller, Ionization of hydrogen by positron impact near the fragmentation threshold, Phys. Rev. A 49 (1994) R4289-R4292.

[244] S. Mori, O. Sueoka, Excitation and ionization cross sections of he, ne and ar by positron impact, Journal of Physics B: Atomic, Molecular and Optical Physics 27 (1994) 4349.

[245] F. M. Jacobsen, N. P. Frandsen, H. Knudsen, U. Mikkelsen, D. M. Schrader, Single ionization of he, ne and ar by positron impact, Journal of Physics B: Atomic, Molecular and Optical Physics 28 (1995) 4691.

[246] D. J. Murtagh, M. Szluinska, J. Moxom, P. V. Reeth, G. Laricchia, Positron-impact ionization and positronium formation from helium, Journal of Physics B: Atomic, Molecular and Optical Physics 38 (2005) 3857.

[247] D. J. Murtagh, D. A. Cooke, G. Laricchia, Excited-state positronium formation from helium, argon, and xenon, Phys. Rev. Lett. 102 (2009) 133202.

[248] G.P. Karwasz, Positrons - an alternative probe to electron scattering, Eur. Phys. J. D 35 (2005) 267-278.

[249] G. P. Karwasz, R. S. Brusa, D. Pliszka, Energy scale determination and energy resolution in positron total cross section measurements, J. Phys. Conference Series 199 (2010) 012019.

[250] P. Caradonna, J. P. Sullivan, A. Jones, C. Makochekanwa, D. Slaughter, D. W. Mueller, S. J. Buckman, Excitation of the $\mathrm{n}=2$ states of helium by positron impact, Phys. Rev. A 80 (2009) 060701.

[251] S. K. Adhikari, A. S. Ghosh, Positron-helium scattering at medium energies, Chem. Phys. Lett. 262 (1996) 460.

[252] L. J. Uhlmann, R. G. Dall, A. G. Truscott, M. D. Hoogerland, K. G. H. Baldwin, S. J. Buckman, Electron collisions with laser cooled and trapped metastable helium atoms: Total scattering cross sections, Phys. Rev. Lett. 94 (2005) 173201.

[253] M. E. Lagus, J. B. Boffard, L. W. Anderson, C. C. Lin, Cross sections of elctron excitation out of metastable helium levels with a fast metastable target produced via charge exchange, Phys. Rev. A 53 (1996) 1505-1518.

[254] G. A. Piech, J. E. Chilton, L. W. Anderson, C. C. Lin, Electron excitation out of the $2^{3} \mathrm{~S}$ metastable level of $\mathrm{He}$ into high- $n$ triplet levels, J. Phys. B 31 (1998) 859-872.

[255] D. V. Fursa, I. Bray, Electron-impact total ionisation of the helium metastable $2^{3} \mathrm{~S}$ state, J. Phys. B 36 (2003) 1663.

[256] T. S. Stein, M. Harte, J. Jiang, W. E. Kauppila, C. K. Kwan, H. Li, S. Zhou, Measurements of positron scattering by hydrogen, alkali metal, and other atoms, Nucl. Instr. and Meth. in Phys. Res. B 143 (1998) 68-80.

[257] J. Mitroy, M. W. J. Bromley, Generating phase shifts from pseudostate energy shifts, Phys. Rev. Lett. 98 (2007) 173001.

[258] E. Surdutovich, M. Harte, W. E. Kauppila, C. K. Kwan, T. S. Stein, Measurements of positronium formation cross sections in positron-mg collisions, Phys. Rev. A 68 (2003) 022709.

[259] C. Cheng, Y. Zhou, Optical model for the positronium formation in positron-mg collision, Phys. Rev. A 73 (2006) 024701. 
[260] I. Bray, Calculation of electron impact total, ionization, and non-breakup cross sections from the $3 S$ and $3 P$ states of sodium, Phys. Rev. Lett. 73 (1994) 10881091.

[261] J. W. Humberston, P. V. Reeth, M. S. T. Watts, W. E. Meyerhof, Positron - hydrogen scattering in the vicinity of the positronium formation threshold, J. Phys. B 30 (1997) 2477.

[262] J. W. Humberston, P. Van Reeth, 2015. Private communication.

[263] J. Mitroy, Formation of antihydrogen by the chargetransfer reaction, Phys. Rev. A 52 (1995) 2859-2864.

[264] J. Mitroy, A. T. Stelbovics, Formation of antihydrogen from antiproton collisions with positronium, J. Phys. B 27 (1994) L79.

[265] J. P. Merrison, H. Bluhme, J. Chevallier, B. I. Deutch, P. Hvelplund, L. V. Jørgensen, H. Knudsen, M. R. Poulsen, M. Charlton, Hydrogen formation by proton impact on positronium, Phys. Rev. Lett. 78 (1997) $2728-2731$.

[266] C. M. Rawlins, A. S. Kadyrov, A. T. Stelbovics, I. Bray, M. Charlton, Calculation of antihydrogen formation via antiproton scattering with excited positronium, Phys. Rev. A 93 (2016) 012709.

[267] J. Mitroy, Positronium-proton scattering at low ener- gies, Aust. J. Phys. 48 (1995) 893-906.

[268] J. Mitroy, Large basis calculation of positron-hydrogen scattering at low energies, Aust. J. Phys. 48 (1995) 645-675.

[269] P. Comini, P.-A. Hervieux, $\overline{\mathrm{H}}^{+}$ion production from collisions between antiprotons and excited positronium: cross sections calculations in the framework of the gbar experiment, New J. Phys. 15 (2013) 095022.

[270] I. Bray, D. A. Konovalov, I. E. McCarthy, Coupledchannel optical calculation of electron-hydrogen scattering from 0.5 to $30 \mathrm{eV}$, Phys. Rev. A 43 (1991) 58785885 .

[271] I. Bray, I. E. McCarthy, Spin-dependent observables in electron-sodium scattering calculated using the coupledchannel optical method, Phys. Rev. A 47 (1993) 317326.

[272] I. I. Fabrikant, A. W. Bray, A. S. Kadyrov, I. Bray, Near-threshold behavior of positroniumantiproton scattering, Phys. Rev. A 94 (2016) 012701.

[273] A. S. Kadyrov, J. J. Bailey, I. Bray, A. T. Stelbovics, Two-center approach to fully differential positron-impact ionization of hydrogen, Phys. Rev. A 89 (2014) 012706.

[274] C. Arcidiacono, A. Kövér, G. Laricchia, Energy-sharing asymmetries in ionization by positron impact, Phys. Rev. Lett. 95 (2005) 223202. 